\title{
Index of Abstract Authors
}

Abbott, Daniel W ....... 329, 845, 1769

Abbott, Sara .................................. 244

Abbruzzese, Mark ......................... 1562

Abdel-Aal, Wafaa E ...................... 945

Abdelal, Heba A ................................. 99

Abdelkader, Amrou .... 621, 845, 1770

Abdelmalek, Manal...................... 1701

Abdi, Jahangir ............................ 1537

Abdulfatah, Eman ....... 113, 139, 175, 1092, 1093, 1094, 1131

Abdulkareem, Fatimah B ............. 767

Abdulla, Farah................................ 512

Abdulrahman, Ahmed A............. 1095

Abdulsattar, Jehan A .................... 2126

Abedalthagafi, Malak................... 1715

Abedi-Ardekani, Behnoush......... 846,

1821

Abel, E Jason ............... 979, 980, 981

Abel, Gyorgy.................................. 2021

Abel, Haley ……………................. 498

Abendroth, Catherine S................. 347

Aberman, Zachary....................... 1363

Abeykoon, Jithma …………….... 1396

Abida, Wassim ................................ 916

Abi Raad, Rita............... 330, 331, 345

Aboelnazar, Nader...................... 1914

Abouelfadl, Dalia .............................. 99

Aboukameel, Amro ......................... 739

Abou Ouf, Hatem........................... 930

Abraham, Jerrold L ….................. 1985

Abraham, Susan ................ 684, 2128

Abraira, Laura ............................. 1748

Abramson, Vandana ........................ 164

Abrial, Catherine............................. 248

Abro, Schuharazad ................ 332, 333

Abu-Farsakh, Sohaib............ 622, 623

Abugharib, Ahmed E ................. 1049

Abulsayen, Hend..... 847, 1361, 1393,

$1412,1480,1882$

Abu-Rustum, Nadeem R ............ 1144

Abu Saadeh, Feras ....................... 1860

Abu Shahin, Nisreen ..................... 1096

Abu-Sheasha, Ghada...................... 152

Abu Zeinah, Ghaith...................... 1349

Abuzenadah, Adel ........................... 627

Accola, Molly .............. 29, 619, 2117

Ackerman, Michael J ..................... 310

Acosta, Andres .................. 624, 1179

Acosta Gonzalez, Gabriel ............. 647

Acree, Rachel............................... 1874

Adam, Benjamin ............... 1287, 1914

Adam-Granger, Eloise................ 2106

Adams, Emily M............................. 403

Adams, Kristen ............................. 847

Adams, Scott............................... 1105

Adams, Thomas A........................... 100

Adebayo, Luqman.............................. 13

Adelaja, Oluwatobi ..................... 2104

Adem, Patricia............................... 2147

Adeniran, Adebowale.......... 330, 331,

345,349

Adeyinka, Adewale ...................... 257

Aditya, Bardia .................................. 164

Adochite, Ramona-Cosmina ...... 2108

Adowanka, Aisha N ..................... 767
Adsay, Volkan ..... 382, 417, 432, 671, $714,756,784,787,837,1773$, 1774, 1778, 1786, 1794, 1795, 1799, 1800, 1804, 1807, 1808

Aejaz, Nasir.................................. 750

Aesif, Scott W .................... 605, 1999

Afify, Alaa ...................................... 387

Afkhami, Michelle ....................... 492

Agaimy, Abbas ........ 69, 84, 848, 849, $984,1065,1282,1338$

Agander, Tina K......................... 1284

Agaram, Narasimhan P ………….... 33

Agarwal, Archana M.................. 1382

Agarwal, Indu .................................. 101

Agarwal, Jai Prakash................... 1289

Agarwal, Nitin K......................... 1532

Agarwal, Shweta ................................ 358

Agarwal, Suresh .......................... 2114

Agboola, AOJ................................. 252

Aggarwal, Nidhi..... 1347, 1425, 1540

Aghajanian, Carol ......................... 1144

Agoff, Nicholas............................ 1226

Agosto-Arroyo, Emmanuel........ 2038

Agoston, Agoston.......................... 1809

Agrawal, Neha .............................. 829

Agrawal, Tanupriya........................ 1771

Agro, Evangeline …..................... 2151

Aguilar-Jakthong, Josephine S.... 334,

1225
734

Aguiluz, Angela ............................. 734

Ahan, Ayse ………........................ 1238

Ahlquist, David A............................ 755

Ahlstedt, Jeffrey ................. 335, 1747

Ahmad, Amar.................................. 861

Ahmad, Farhan............................. 1389

Ahmed, Atif...................................... 85

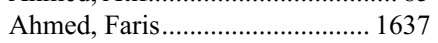

Ahmed, Ishtiaque .......................... 1587

Ahmed, Shahida ........................... 1427

Ahn, Sangtae..................... 1881, 2162

Ahn, Soomin ......................................... 636

Ahram, Mamoun .......................... 1096

Ahsanuddin, Arshad ..................... 1483

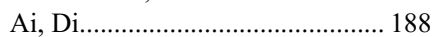

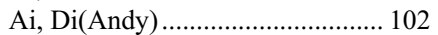

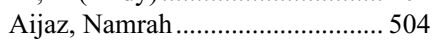

Ailles, Laurie............................... 1275

Ainechi, Sanaz ...... 1005, 1654, 1881,

2162

Aird, John.................................. 1614

Akazawa, Yuko ................. 1958, 2142

Akhtar, Israh....................... 412, 2024

Akhter, Shabnam.............. 1098, 1099

Akin-Akintayo, Oladunni O......... 998

Akinde, Olakanmi R ....................... 13

Akki, Ashwin ................... 2016, 2074

Aksentijevich, Ivona .................... 1358

Alaghehbandan, Reza ......... 849, 983,

1054

Al-Ahmadie, Hikmat........... 850, 874, 916, 935, 985, 1037, 1038, 1053, $1055,1058,1910$

Al-Ahwal, Mahmoud S................ 627

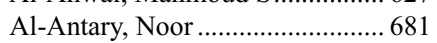

Alatassi, Houda ........... 493, 855, 1858
Alattia, Lubna A............... 2017, 2105 Al-Awar, Shamsa ........................ 1223

Albadine, Roula ............................. 2106

AL Badri, Osamah ....................... 1716

Albain, Kathy S............................ 1907

Albanese, Christopher................. 1874

Albanese, Joseph............... 1394, 1839

Al-Baqer, Tahani ......................... 1984

Albarracin, Constance T ............ 1846 ,

2128

Albawardi, Alia ............................. 1223

Alberts, Steven R ............................ 722

Al-Bozom, Issam .......................... 1008

Albustanji, Amani ........................ 1096

Alcasid, Matthew ……………….... 1471

Alchami, Fouad S........................ 1980

Aldayel, Fouad.............................. 494

Aldecoa, Iban .......... 625, 1348, 1893,

2139

Alekseev, Boris ............................. 1845

Alex, Deepu .............. 668, 1896, 1953

Alexander, Erik K .......................... 614

Alexander, Mariam P ...... 1615, 1626 ,

1643,1652

Alexander, Melissa.......................... 103

Alexander, Rhonda............................... 547

Alexandrescu, Sanda........ 1720, 1746

Alexiev, Borislav............................ 577

Alexis, John.................................. 1918

Al-Ghamdi, Fahad...................... 1717

Alghamdi, Mohammed A... 493, 1858

Al-Ghamdi, Yahya ...................... 1283

Al Ghuzlan, Abir.................. 473, 601

Al Hmada, Youssef ...................... 1718

Alholle, Abdullah ............................ 87

Ali, Siraj M ........ 196, 238, 496, 790, 994, 1005, 1015, 1297, 1331, $1654,1836,1855,1880,1920$.

1924

Ali, Syed Z ......... 336, 374, 390, 402,

411,2066

Al-Ibraheemi, Alyaa......... 31, 39, 500

Ali-Fehmi, Rouba ........ 113, 139, 175, $1092,1093,1094,1131$

Alkan, Serhan................................ 1623

Al-Kawaaz, Mustafa ..................... 1349

Alkhader, Ali ................................. 1096

Alkhasawneh, Ahmad .......... 20, 1568

AL-Khinji, Aisha.......................... 1380

Allan, John ................................... 1349

Al-lbraheemi, Alyaa ......................... 74

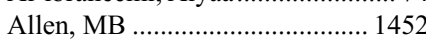

Allen, Peter .................................... 1773

Allen, Richard A............................ 1448

Allende, Daniela ......... 382, 409, 543, $654,705,1655$

Allgaeuer, Michael........................ 626 Allison, Derek ............ 402, 403, 1772

Allison, Kimberly H....111, 112, 2032

Al-Maghrabi, Basim J.......... 627, 851

Al-Maghrabi, Jaudah A........ 627, 851,

$\begin{array}{rr} & 1717 \\ \text { Almardini, Nour } & 917\end{array}$

Almarzooqi, Saeeda SH ............... 1223

Almekinders, Mathilde ........ 134, 135

Almenara, Jorge A................ 433, 1976

Al-Moustafa, Ala-Eddin................ 681
Almutairi, Fawaz............................ 860 Alnajar, Hussein ................ 1616, 1981

Al-Nourhji, Omar......................... 1100

Al-Obaidy, Khaleel I........ 1092, 1897

Alobeid, Bachir................. 1478, 1489

Alos, Llucia.......................................... 359

Alos, Silvia....................................... 359

Alosh, Baraa...... 113, 139, 175, 1092,

1131

Alpers, Charles E ......................... 1651

Alpert, Lindsay ........... 628, 629, 719,

1656,1673

Al-Qahtani, Mohammad ............ 1717

Al-Qahtani, Mohammed H ........... 627

Alqassab, Usama.............................. 995

Alqatari, Mahfood....................... 1198

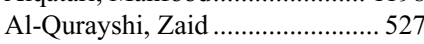

Alrajjal, Ahmed............................ 1634

Al-Rohil, Rami..................... 513, 2018

Alruwaii, Fatimah I............ 852, 1898

Alsadi, Alaa................................... 1604

Alshalalfa, Mohammed...... 930, 1056

Alsolami, Afaf................................ 494

Altiok, Soner ........................ 50, 1843

Altorki, Nasser K .............. 1937, 1938

Altree-Tacha, David ....................... 2107

Al-Turkmani, MRabie................. 1052

Alva, Ajjai ........................ 1049, 1069

Alvarado-Cabrero, Isabel.......... 1028, $1029,1101,1244,1245$

Al-Zaid, Tariq.......................... 32, 494

Amacher, Anne Marie O ............ 1350

Amador, Lucia .................................. 303

Amano, Atsushi.............................. 317

Amaya, Clarissa N .......................... 91

Ambaye, Abiy ..................... 665, 2034

Ambelil, Manju ................................ 630

Ambrosone, Christine B.............. 1907

Amemiya, Yutaka......231, 1158, 1159

Ameri, Maryam D............ 1545, 2102

Amin, Ali........................... 917, 2108

Amin, Hebatallah A.......................... 99

Amin, Khalid....... 418, 437, 449, 450, $484,606,2076,2085$

Amin, Mahul B ... 848, 892, 914, 969,

1038,2133

Amin, Said M.................................. 767

Amini, Behrang.................................... 34

Amini, Elham........................ 827, 828

Amrollahi, Fatemeh ..................... 1760

An, Soyeon................................... 1790

Anandan, Vasuki ........................... 292

Ananthanarayanan,

Vijayalakshmi

1578,1600 ,

1980, 1981

Andeen, Nicole ................... 513, 1062

Anders, Robert A.... 1688, 1706, 1707 Anderson, Blake B .............. 876, 877

Anderson, Joshua ........................ 1351

Anderson, Kevin ............................ 2019

Anderson, Matthew...................... 1203

Anderson, Michele........................ 1873

Anderson, Neil ............................... 1557

Anderson, Scott.............................1115

Andion, Maitane ........................... 1409

Andrade, Priscila................................. 368

Andrade, Rafael E....................... 1401 
Andras, Kapus................................... 963 Andre, Saudade .................... 249, 367 Andreasen, Simon .... 517, 1284, 1292 Andreev, Gregory........................ 2108 Andreev, Oleg A........................... 2108 Andreoli, Steven ........................... 1889 Andreopoulou, Eleni.... 126, 127, 128 Andres, Maria del Mar................ 1409 Andresen, Kevin ......................... 1712 Andreu, Xavier................................ 223 Andrici, Juliana .............................. 232 Andrulis, Irene ........... 155, 280, 2158 Aneja, Amandeep .......................... 1343 Angeletti, Cesar............................ 2109 Angelova, Evgeniya A ..... 1352, 1353 Angot, Émilie ............................. 1972 Anichini, Andrea ............................ 595 Anis, Shady E.................................. 945 Annan, Anand C......... 104, 997, 2110 Annunziata, Joseph F........ 339, 1285,

1822,2111

Ansari, Junaid ............................... 2105

Ansari, Mohammad Q................ 2021

Ansell, Stephen M....................... 1396

Ansell, Wendy ................................ 1042

Antic, Tatjana ....... 338, 354, 415, 416, 483, 906, 907, 966, 975, 984,

1066,1255

Antonescu, Cristina R ....... 33, 55, 56, $87,1125,1282,1910$

Ao, Minghui .................................. 1850

Aoki, Ichiro ...................................... 58

Aoun, Patricia A.............................. 492

Appelman, Henry D ... 689, 726, 2133

Apple, Sophia........................ 105, 106

April, William ............................. 1767

Aqil, Barina .................................... 569

Arab, Seyedeh Elham........... 336, 411

Arana, Iñigo .................................. 1739

Aranake-Chrisinger, John ............... 34

Aranda, FI ……………………..... 159

Araujo, Karina S .......... 862, 863, 864

Araya, Camila B........................... 1533

Araya, Juan Carlos ............ 784, 1774, $1795,1804,1808$

Arber, Daniel A..... 1384, 1398, 1437, $1452,1471,1472$

Arcaro, Kathleen F...................... 1841

Arciero, Cletus ................................ 218

Arcila, Maria E........ 874, 1298, 1831, $1837,1896,1953$

Arciuolo, Damiano...................... 1277

Aretini, Paolo ....................................... 66

Argani, Pedram ... 124, 266, 281, 282,

$297,961,2133$

Argenyi, Zsolt B............................. 513

Argyris, Prokopios ...................... 1286

Arispe, Karen .............................. 1672

Aristi Urista, Gerardo..................... 669

Ariza, Aurelio....................................... 757

Armanious, Hanan ............ 1287, 2112

Armengol, Carolina....................... 1875

Armenia, Joshua..............................916

Armylagos, Donna ........ 370, 380, 440

Arnason, Thomas .......................... 738

Arnold, Christina A..... 551, 655, 746, $796,803,806,1888$

Arnold, Michael A...... 551, 803, 1888

Arnold, Rebecca............................. 995

Arnould, Laurent........................... 1128

Aron, Manju..

1075
Arora, Kshitij S .......... 631, 708, 765, 1266

Arora, Samriti ....................... 35, 1991

Arora, Taranjit K ............................... 197

Arpin, Ronald....................... 378, 438

Arrossi, Andrea V ....................... 1955

Arthur, Gaba.................................. 410

Arun, Indu ..................................... 794

Arva, Nicoleta .............................. 1877

Asa, Sylvia L....................... 579, 582

Asada, Yujiro................................ 1632

Asaka, Shiho .................... 1102, 1899

Asamura, Hisao.............................. 1954

Asato, Marcel A ........... 862, 863, 864

Ascani, Stefano ........................... 1750

Asch-Kendrick, Rebecca...... 124, 282

Ashford, Bruce....... 1305, 1330, 1333

Ashi, Shaymaa AL-L................... 1617

Ashikaga, Takamaru....................... 666

Ashman, Dayne.............................. 337

Ashraf, Nabil........... 433, 1288, 1976

Asiry, Saeed ................................. 2077

Askan, Gokce.................. 1773, 1807

Askari, Reza............................... 1576

Askin, Frederic................................... 1850

Asleh-Aburaya, Karama ............. 1253

Assaad, Adel ................................... 1300

Assarzadegan, Naziheh ................ 673

Assidi, Mourad............................... 627

Assylbekova, Binara ........... 141, 209,

1103, 1258

Astigarraga, Itziar ....................... 1409

Astles, JR ........................ 2068, 2069

Astvatsaturyan, Kristine........ 107, 108

Aswad, Bassam ................ 1917, 1968

Atenafu, Eshetu G.......... 1184, 1372,

1432,1487

Athanazio, Daniel ............... 867, 1065

Atieh, Mohammad .......................... 445

Atkins, Kristen ......... 146, 1252, 1276

Atkinson, James .............................. 323

Atsaves, Vasillios .......................... 1354

Atta, Mohamed Adel ...................... 898

Attanoos, Richard ....................... 1980

Attiyeh, Marc ................................ 1861

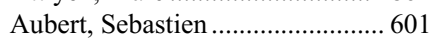

Aubertin, Kelly ................ 2125, 2152

Aubry, Marie C ...... 1929, 1956, 1971

Aubry, Marie Christine ............... 2004

Aucejo, Federico .......................... 1655

Audenet, François ............................ 850

Auerbach, Aaron .......................... 1873

Auger, Cristina ............................... 1748

Aung, Kimberly …......................... 123

Aung, Kyaw .................................. 1835

Aung, Phyu P .... 525, 533, 537, 1355,

1758,2018

Aust, Jr., Charles H ...................... 2009

Autio, Karen A ................................ 935

Avadhani, Vaidehi ....... 417, 432, 632,

1657

Aviel-Ronen, Sarit............ 1244, 1245

Avkshtol, Valentina ............... 148, 149

Avogbe, Patrice H ....................... 1821

Avril, Stefanie ............................... 187

Aw, Sze Jet ................................... 1869

Awadallah, Amad ..... 385, 1740, 1779

Awasthi, Namrata P..................... 1166

Axiotis, Constantine A .................. 583

Ayala, Alberto ................. 36, 224, 328

Ayangbesan, Abimbola ................. 978
Ayers, Leona W............................ 1601

Aynardi, Jason ............................... 1356

Ayoola-Adeola, Martins.............. 1192

Azad, Azar.......................................... 1070

Azad, Sassan ................................ 1984

Azar, Azniv .................................. 1718

Azevedo, Livia LA....... 862, 863, 864

Azevedo, Paulo H .......................... 138

Azevedo de Andrade,

Tathiana..... 1380

Azevedo Neto, Raymundo ......... 1450,

Azkargorta, Mikel ........................ 1875

Azmi, Asfar .................................... 739

Azordegan, Nazila........................ 1250

Azorín, Daniel.................................... 1409

Azria, David.................................. 248

Azzato, Elizabeth .......................... 1890

Babu, KM........................................... 648

Babushok, Daria.......................... 1356

Bacani, Julinor ................................ 814

Bacchi, Carlos E............................. 984

Backes, Floor J............................. 1192

Badmos, Kabir ............................... 528

Badrinarain, Jason.........................1110

Badve, Sunil............... 109, 163, 1978

Bae, Hyunsik................. 130, 633, 659

Bae, Ja Seong .................................. 584

Bae, Young Kyung .......................... 204

Baecklund, Eva .............................. 1526

Baeesa, Saleh ............................. 1717

Baena Del Valle, Javier A... 853, 2113

Baergen, Rebecca........................ 1887

Bagg, Adam............ 1356, 1384, 1493

Baggerly, Keith ............................ 883

Baglieri, Jacopo............................. 1686

Bahar, Burak ................................ 2020

Bahirwade, Ganesh ....................... 634

Bahler, David W.............................. 1386

Bahmanyar, Mohammad ............ 1487

Bahrami, Armita...................... 44, 495

Bai, Isaac.................................... 2114

Bai, Shi......... 1357, 1407, 1408, 1423

Bailey, Jennifer M........................ 1819

Bailey, Mark A ............................. 2025

Bailey, Nathaniel................................. 1460

Baine, Marina K................ 1104, 1900

Bajaj, Mandeep S ......................... 1335

Bajorin, Dean F......... 850, 985, 1055 ,

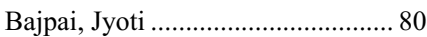

Baker, Gabrielle ................... 110, 284

Baker, Jeryl .................................... 102

Baker, Tiffany ............................... 1719

Bakhshi, Sameer ........................... 1335

Bakkum-Gamez, Jamie N .......... 1270

Bal, Munita ........... 1289, 1311, 1324

1797

Balagué, Olga.................... 1348, 1409

Balaguer, Francesc ............... 625, 2139

Balakrishna, Jayalakshmi P........ 1358

Balassanian, Ronald.................... 1569

Balatico, Michael A..................... 1978

Balci, Serdar.............. 756, 837, 1773

Baldaia, Helena .............................. 1773

Baldion, Ana Margarita............... 1574

Bale, Tejus A ................................. 1720

Balk, Steven P..................... 873, 987

Balla, Andre …….................... 2104

Ballard, Morgan .....................111, 112

Ballen, Karen

1381
Ballentine, Samuel J....................... 854

Ballester, Hortensia ......................... 158

Ballester, Leomar .......................... 1721

Ballinger, Lori .................................. 49

Baloch, Zubair....................... 490, 582

Balogh, Zsofia ................................. 473

Baltaro, Richard J........................ 2021

Baltatzis, George E ..................... 1492

Baltay, Michele ………………..... 1847

Baltay, Michele T ............. 1242, 1546

Balzer, Bonnie.............. 504, 505, 536

Banach, Bridget S ....................... 338

Bandoh, Shuji................................. 1936

Bandy, Andrew................................ 635

Bandyopadhyay, Sudeshna ......... 113, 139, 175, 1092, 1093, 1094, 1131

Banerjee, Anjishnu .............. 845, 2131

Banerjee, Diponkar ....................... 2091

Banerji, Nilanjana ..........................593

Banet, Natalie.............................. 1272

Bang, Heejin ................................... 636

Bangash, Mohammed ................. 1717

Bangs, Charles D .............................. 1365

Bangs, Dana .................................. 1939

Baniak, Nick ................................ 1105

Banizs, Anna ...339, 1285, 1822, 2111

Banjo, Adekumbiola ..................... 528

Banks, Peter ................. 180, 212, 213

Banks, Roz .............................................. 846

Banky, Sandra ................................ 1602

Bann, Darrin............................ 612, 613

Bansal, Meenakshi G ................. 2022

Bantz, Christopher ..................... 2082

Baraban, Ezra ................................ 1359

Barahona, Marc............................ 1229

Baral, Reetu................................. 1237

Baras, Alexander ........ 941, 951, 1060

Baratta, Paul.................................... 123

Barbieri, Andreia.................. 36, 1729

Barbuto, Denise............................ 1093

Bárcena, Carmen ........................... 1409

Barkan, Guliz ...... 332, 333, 420, 427, $428,443,444,445,464,957,968$

Barkan, Guliz A.............................. 586

Barker, Heather S ........................... 855

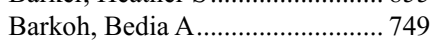

Barletta, Justine A ................. 607, 614

Barlogie, Bart................................ 1377

Barna, Nicholas.............................. 856

Barnard, Nicola ............................ 1605

Barnes, Michael .............................. 2114

Barnhill, Raymond .......................... 495

Barrera-Herrera, Luis E ................ 587

Barreto, Jose................................. 1017

Barrett, Amanda ............................... 857

Barretta, Francesco ......................... 595

Barr Fritcher, Emily .................... 1808

Barr Fritcher, Emily G ............... 2010

Barrows, Brad D ................ 637, 1658

Barry, Marc .................................... 1293

Barry-Holson, Keegan ................ 1106

Barry O'Crowley, Jacqui .............. 435

Barsan, Iulia ..................... 1244, 1245

Bartlett, Nancy ............................ 1496

Barua, Ashim R ............................... 1098

Baselga, Jose .................................... 165

Basha, Basma ................................... 1360

Bashir, Samir Al ........................... 1065

Bashleben, Christine .................... 2021

Bashover, Eva M........................... 114

Bassat, Quique ............................ 1573 
Basset, Roland.................................. 472

Bassett, Roland ............................. 125

Bassiouny, Dina .................1160, 1161

Basso, Cristina ..................... 321, 322

Bastacky, Sheldon ........................ 1034

Bastos, Lucas QA.......... 862, 863, 864

Basturk, Olca.................... 1773, 1807

Bates, Gleneara ........................... 1967

Bates, Michael................................ 232

Baudhuin, Linnea M ..................... 310

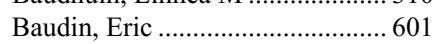

Baum, Jordan E.................. 115, 1823

Baumgartner, Joel M..................... 818

Baunoch, David................................. 678

Baus, Rebecca ………………….... 591

Bautista, Pinky ........................... 1585

Bavi, Prashant ............ 638, 639, 1789

Bawab, Ramzi ............................. 1891

Bayat, Sahar ........................ 947, 948

Bayol, Beryl ............................... 1107

Bean, Gregory .............116, 199, 1108

Bean, Gregory R .............................. 37

Beaubier, Nike T ........ 674, 711, 1842

Beca, Francisco .............................. 117

Bechert, Charles............................. 2114

Beck, Andrew H.................. 117, 121

Beck, Janina ................................. 1492

Becker, Renato ............................. 1774

Bedard, Karen .................................. 738

Bedolla, Gabriella ............. 1795, 1799

Bedroske, Patrick P............ 689, 1971

Beech, Cameron ............................. 340

Beer, Tomasz M ............................ 934

Behm, Frederick................. 2148, 2150

Behrens, Carmen ............................. 749

Behtaj, Mohadese.......................... 640

Belanger, Eric C........................... 1048

Belaud-Rotureau,

Marc-Antoine....... 946, 948, 1444

Belchis, Deborah............................... 43

Belendez, Cristina ......................... 1875

Belinson, Jerome L ..................... 1280

Bell, Debra A..................... 1278, 2008

Bell, Diana ........................ 1290, 1300

Bell, Douglas................................ 1612

Bellamy, William ......................... 2134

Bellizzi, Andrew M............. 585, 588, 641, 1438, 1687, 1764, 1781,

$1884,2023,2088$

Bellolio, Enrique... 1774, 1795, 1804,

1808

Belousova, Irena E ........................ 521

Beltran, Himisha ....... 882, 1853, 1857

Beltrán, Javier G ............................ 355

Beltran, Luis.......................... 858, 861

Benasalah, Karim................ 946, 947

Benayed, Ryma ................ 1298, 1837

Benedetti, Ines................................ 993

Benevides Guedes, Liana..... 859, 860

Ben-Gasheer, Mohammad........... 1008

Benini, Stefania................................. 47

Bennett, Jennifer .............................. 1110

Bensalah, Karim............................ 948

Berezowska, Sabina .................... 1959

Berg, Aaron N ............................... 425

Berger, David ................................ 1879

Berger, Michael F...... 850, 916, 1055,

1058,1298

Bergeron, Anne ............................ 1950

Bergfeld, Wilma F.......................... 534

Bergstrom, Colin .......................... 1135
Bergum, Christopher..................... 292

Berman, David ........... 256, 867, 2145

Berman, Samuel H.................... 1854

Bernardini, Marcus ...........1180, 1184,

Bernardo, Margarita ..................... 740

Bernatsky, Sasha ......................... 1526

Berney, Daniel..... 848, 849, 858, 861, 1042

Bernhard, Jean-Christophe.......... 946, 947,948

Berni, Silvia ................................ 1723

Bernicker, Eric .............................. 1912, 1913

Bernieh, Anas ................... 1361, 2024

Berrocal, Julian ............................. 299

Bertherat, Jerome ............................ 601

Bertolotti, Alessia.......................... 879

Bertsch, Elizabeth C....................... 341

Bertz, Simone................................... 881

Beseler, Katie ................................ 1588

Beshai, Boulos ..................................... 1

Betman, Shane ............................. 1606

Bettington, Mark ................. 728, 793

Betz, Bryan .................................. 868

Bevilacqua, Generoso ...................... 66

Beydoun, Rafic............. 739, 740, 741

Bezerra, Stephania M....... 1018, 1059

Bezieau, Stéphane ......................... 646

Bhagat, Govind ................ 1478, 1489

Bhaijee, Feriyl..................................... 761

Bhalla, Amarpreet .......................... 657

Bhan, Atul .................................... 2101

Bhandari, Vijendra .......................... 35

Bhardwaj, Mansi .......................... 1335

Bhardwaj, Nitin............................ 986

Bhargava, Rohit .......... 140, 141, 182, 209, 230, 1103, 1174, 1258

Bhaskaran, Archana .................... 2076

Bhat, Rukhmi ............................. 1453

Bhattacharjee, Meenakshi .............. 27

Bhattarai, Ava ................................ 641

Bhavsar, Shweta......................... 1425

Bhimji-Pattni, Sonal........... 104, 2110

Bhuiya, Tawfiqul A ......................... 168

Bhullar, Jasneet S .......................... 292

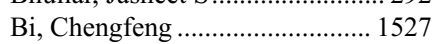

Bi, Rui ...............................279, 1111

Biagi, James J.................................. 643

Bibars, Wafi........................ 1775, 1827

Bibbey, Scott .................................... 342

Bibbo, Marluce ……………........... 381

Bidart, Jean-Michel....................... 473

Bidet, Yannick ............................... 1107

Biernacka, Anna ............................. 118

Biffoni, Marco................................. 1723

Bignon, Yves-Jean....................... 1107

Bilawich, Ana-Maria ................... 1946

Bilir, Birdal ............................. 997, 998

Billings, Steven ..... 46, 500, 518, 519,

530

Billis, Athanase ............ 862, 863, 864

Bilodeau, Elizabeth A.................. 1286

Bilotta, Mirna ................................. 439

Binte Md Nasir, Nur Diyana ........ 277

Birkness, Jacqueline....................... 642

Bischoff, Farideh............................ 123

Biscotti, Charles V ......................... 1100, 1280

Bishop, Barbara............................ 1393

Bishop, Julie Anne ........................ 119

Bishop, Justin A ........ 374, 913, 1282, 1291, 1292, 1302, 1319, 1338
Bishp, Justin A ................................ 402

Bismar, Tarek A............................. 930

Biswas, Asok ................................. 503

Bitterman, Pincas ............. 1235, 1308

Bitting, Angela ............................. 656

Bivalacqua, Trinity J ......... 1018, 1060

Bivin, William W ........................ 2053

Bixby, Dale ................................... 1878

Bizzarro, Tommaso .... 439, 490, 1277

Black, Dalliah M........................ 2025

Black, Margaret A ............................. 649

Blackwell, Rebecca..................... 2138

Blakely, Morgan.............................1112

Blakesley, Rick …….................... 1121

Blanco, Luis Z.............. 166, 288, 289

Blanco, Paula .............................. 1722

Blanes, Alfredo …............... 578, 1833

Bledsoe, Jacob R.................. 789, 1513

Blessing, Melissa M......................... 38

Blevins, Allen R .............................. 123

Block, Norman L........................... 194

Bo, Ping............................... 336, 411

Boardman, Lisa ............................. 1666

Boccardo, Simona ........................ 1923

Bochaton-Piallat, Marie-Luce...... 321

Bochner, Bernard H ............ 850, 985,

1055,1058

Bocicariu, Alina ............................ 643

Bocklage, Therese................ 49, 1293

Bodo, Juraj ....................................... 1414

Boehm, Steffen............................ 1151

Boerner, Julie ................................... 113

Boerner, Scott L ............................ 466

Boers, James .................................. 2115

Bogardus, Sidney ........................... 652

Bogdanska, Anna ........................ 1860

Boguniewicz, Ann........................... 132

Bohm, Maret ............................... 1603

Boiocchi, Leonardo............................. 1362

Bois, Melanie ............... 309, 310, 311

Bojanowski, Christine M ........... 1947

Bokhari, Aqiba ................................. 675

Boland, Jennifer M......... 39, 44, 1680 , 1786, 1971, 1986, 1999

Boland, Patrick................................. 79

Bolesta, Edward ............................. 572

Bolger, Noel .................................. 435

Bollen, Andrew …….................... 1753

Bolognesi, Maddalena M............ 2136

Boman, Bruce ............................... 685

Bombi, Josep Antoni............ 625, 2139

Bombonati, Alessandro ................. 644

Bondaruk, Jolanta ............................ 883

Bondzie, Philip A ............................ 832

Bonert, Martin.................................. 544

Bonert, Michael.......... 544, 760, 1580

Bongiovanni, Massimo ................. 570

Bonneau, Peter N ............................. 651

Bonzheim, Irina............................. 1507

Booker, Betty ………......................... 2116

Bookstein, Robert E ....................... 734

Boorjian, Stephen A ......................... 888

Booth, Christine N ...................... 1322

Borah, Sumit ................................. 495

Borbath, Ivan..................................... 478

Borczuk, Alain C...... 810, 1851, 1967

Borer, Jill..................................... 334

Borger, Darrell ............................. 1265

Borhan, Walaa ...................... 865, 1019

Boriani, Stefano ................................ 47

Boroujen, Amir M.

1609
Borowitz, Michael........................ 1555

Borri, Filippo....................................... 644

Borscheid, Rene .......................... 1576

Borsu, Laetitia................................ 1903

Borys, Dariusz.............................. 1600

Borys, Ewa ...................................... 1600

Boscato, Elena.............................. 1750

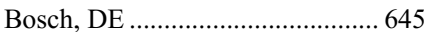

Bosch, Dustin.............................. 1659

Bose, Shikha ........ 107, 108, 611, 892

Boskamp, Tobias .......................... 2157

Bosman, Fred ...................................... 672

Bossard, Céline ................................. 646

Bosse, Tjalling .............................1113

Bossé, Yohan............................. 1987

Bossler, Aaron ........................... 54, 384

Bossuyt, Veerle...................... 133, 545

Bouda, Jiri ...................................... 521

Boukhar, Sarag....... 1480, 1659, 1687

Bouska, Alyssa .............................. 1380

Boutsen, Laure .............................. 478

Bowden, James ............................. 1209

Bowles, Daniel.................. 1297, 1331

Bowman, Sarah ............................ 1829

Bowman, Teresa.................................. 202

Bowser, Zenica............................... 135

Box, Adrian ................................. 1550

Boyce, Alison.................................... 761

Boyden, Edward............................. 121

Boyer, Daniel .................................... 1363

Boyer, Michael.............................. 1330

Boyle, Suzanne M....................... 1617

Bozkurtlar, Emine ....................... 1993

Bracci, Page ................................. 1514

Brachtel, Elena.......... 118, 708, 2028,

2101

Bradshaw, Azore-Dee .......... 647, 695

Braegelmann, Johannes................. 996

Brainard, Jennifer A .... 361, 392, 394,

Brais, Lauren.............................. 1803

Braith, Andrew ................................. 565

Brancely, Dawn..................... 468, 469

Brand, Randall E.................. 467, 1813

Brandler, Tamar C........ 343, 352, 397

Brandwein, Margaret ....... 1341, 1345

Brannigan, Robert E.................... 2123

Brassell, Stephen............................... 871

Brat, Daniel J ........ 1737, 1753, 1759,

1760

Braun, Alexander L.................... 1582

Braunhut, Beth L............... 1618, 1621

Braylan, Raul .................................. 1389

Brcic, Luka................................... 1980

Breau, Rodney H............................... 1048

Bredno, Joerg ................................ 2114

Brennan, Michael......................... 1213

Brennan, Paul .................................... 846

Brennan, Rachel........................... 1890

Brenner, David ....................................... 1686

Breslauer, Cori .................................. 866

Breuer, Frank …………………..... 293

Brickman, Arlen........................... 1604

Bridges, Alina G.............................. 506

Bridgewater, John ........................ 1805

Brimo, Fadi ....... 867, 881, 891, 1024,

106

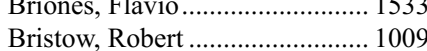

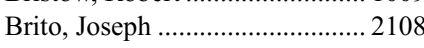

Britt, Jacob

169 
Britton, Heidi .....................1113, 1250

Broaddus, Russell R.... 147, 537, 749, $1114,1129,1130,1148,1149$, $1187,1824,1830,1846$

Brock, Jane........................... 201, 232

Brodie, Caroline ............................. 120

Broehm, Cory................ 31, 39, 1293

Brogi, Edi.... 134, 135, 161, 220, 243 ,

285,286

Brosens, Lodewijk AA.................. 761

Brouwer, Jan ................................. 1243

Brower, Raven .................. 1347, 1443

Brown, Charmaine G .................. 1313

Brown, Ian................... 662, 698, 793

Brown, J Mark ........................... 1655

Brown, Laura ............................... 1364

Brown, Marty E ............................. 123

Brown, Noah....................... 868, 1460

Brown, Ron............................... 1426

Browne, Eva P.............................. 1841

Bruegl, Amanda ...........................1114

Brufsky, Adam M......................... 140

Brunelli, Matteo ............................ 869

Bruner, Andrew ……………......... 250

Brunot, Angelique......... 946, 947, 948

Brunt, Elizabeth M........... 1677, 1695

Brunyé, Tad T.............................. 2032

Bryan, Andrew ………................ 1558

Bryan, Brad A................................... 91

Bryant, Britni RE ...............1115, 2034

Bryant, Madalynn ...................... 1424

Bshara, Wiam............................... 1907

Bsirini, Caroline .................... 870, 887

Bu, Fang ....................................... 1867

Buchanan, Daniel................ 791, 792

Buchert, Gerald ................................ 36

Buckingham, Lela ....................... 1616

Bucur, Octavian ............................. 121

Buechler, Steven A......................... 109

Buehler, Darya .... 602, 605, 618, 619,

1232

Buendia, Marie A ........................ 1875

Bueso-Ramos, Carlos E ............. 1403, $1417,1418,1429,1434,1445$,

1446,1488

Buglioni, Alessia .......................... 1619

Buhmeida, Abdelbaset .................. 627

Buhtoiarova, Tatiana N ................1116

Bukelo, Maria .................................. 648

Bulimbasic, Stela ......................... 984

Bull, Shelley B ................................ 155

Bullock, Martin ............................ 1292

Bullock, Timothy ........................... 146

Bulman, William .......................... 1928

Bundele, Manish M...................... 1294

Bundock, Elizabeth ..................... 1757

Buonocore, Darren ....................... 344

Burack, Richard .... 1385, 1424, 1430,

2022, 2120

Burger, Charles .............................. 1944

Burger, Peter C............................. 1761

Burke, Allen .................................. 1076

Burke, Allen P... 871, 872, 912, 1995,

2015

Burke, Carol......................... 705, 706

Burke, Kathleen A....... 161, 207, 220, $242,243,1152,1312,1854$

Burke, Louise ............................... 1905

Burmeister, Lynn A ............. 450, 2085

Burnier, Miguel N ........... 1731, 1767,

1768
Burns, Kathleen H......................... 1555

Burra, Patrizia ............................. 1685

Burton, Ann.................................... 688

Busam, Klaus J................................ 514

Busca, Aurelia ................................1117

Buschmann, Mary .......................... 338

Butler, Marcus..................... 639, 1185

Butler-Wu, Susan ......................... 1564

Butnor, Kelly.................... 1901, 1980

Butowski, Nicholas .................... 1753

Butz, Henriett................................ 963

Buyukeren Babaoglu, Berrin .......... 61

Buza, Natalia........... 133, 1104, 1224,

1267,1281

Byrne, Michael................. 1504, 1510

Byrnes, Kathleen........... 2, 1557, 1660

Cabanillas, Maria E........................ 455

Cabeza, Eduardo A........................ 1070

Cabezas, Claudia .......................... 1533

Cabrera-Muñoz, Lourdes ............ 1868

Cacciabeve, Nicolas.................... 1583

Cacciatore, Matilde ............... 81, 1750

Cadoo, Karen ................................ 1144

Cagle, Philip T .................. 1912, 1913

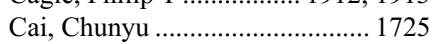

Cai, Donghong ……..................... 1427

Cai, Emily ..................................... 518

Cai, Guoping ....... 330, 331, 340, 345,

1776

Cai, Lei.......................................... 631

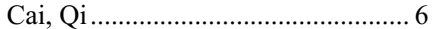

Cai, Weiwei.................................. 1039

Cai, Zhenjian.............. 843, 844, 1779

Caillou, Bernard............................. 473

Cajaiba, Mariana M .................... 1618

Calagua, Carla....................... 873, 987

Caldwell, James ............................... 10

Calhoun, Benjamin C... 122, 148, 149

Caliò, Anna ................................. 869

Calkins, Sarah ................................ 546

Callender, Glenda G....................... 349

Calonje, Eduardo............................. 511

Calvo, Katherine R............ 1358, 1535

Calvo, Maria Teresa R ................. 355

Camacho, Jessica ........................ 1748

Câmara, Sara ................................... 249

Camara-Lopes, Luiz Heraldo A ... 399

Campbell, Katelynn ......................1118

Campillo-Gimenez, Boris ............. 947

Campo, Elias ......... 1348, 1390, 1409

Campo, Meghan J 1475

Campuzano-Zuluaga, Germán ... 1431

Camuso, Natasha.......................... 2091

Can, Nhu Thuy .................................. 546

Canavez, Flavio C.......................... 399

Canavez, Juliana MS...................... 399

Candela, Ninfa M.............................. 704

Canessa, Pier Aldo ....................... 1923

Cañete-Portillo, Sofía...... 1017, 1027,

1028,1029

Cani, Andi .......................................... 123

Cannell, Emily M........................... 123

Canter, Robert J................................96

Cantley, Lewis............................ 1853

Cantu, David .......... 1518, 1545, 2102

Cantu de Leon, David ..... 1002, 1047,

1220

Cao, Dengfeng ......... 839, 1088, 1279

Cao, Weibiao.................................. 1825

Cao, Wenqing

649
Cao, Xuhong ... 497, 590, 1049, 1050, 1069

Capodimonti, Sara........................... 439

Caporelli, Mandi-Lee...................... 461

Caraway, Nancy P .......................... 366

Carballo-Zarate, Adrian A........... 1446

Card, Sydney ......................1119, 1120

Cardona, Diana ........ 693, 1043, 1701

Cardona, Ken ............................... 1804

Carey, John L ......... 1426, 1525, 2043

Carll, Timothy ............................... 2036

Carlson, J Andrew ........................... 496

Carneal, Eugene .................. 1365, 1366

Carneiro, Fatima …....................... 1773

Carney, John................................ 1902

Carpio, Luis...................................... 469

Carr, David...................................... 650

Carr, Richard A ............................... 522

Carrasco, Miquel Angel ................ 757

Carreira, Christine ......................... 1821

Carrilho, Carla.............................. 1573

Carrillo-Polanco, Luis.................... 651

Carroll, Martin ............................... 1544

Carroll, Sarah ...................... 346, 2117

Carskadon, Shannon ...................... 943

Carter, Cody .................................... 497

Carter, Jodi ......................................... 39

Carthon, Bradley C ........................ 927

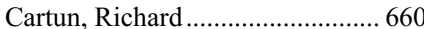

Carty, Sally E ................................. 425

Carusi, Daniela A .......................... 1247

Caruso, Carla.................................... 347

Carvalho, Paulo A .......................... 959

Casadei, Riccardo ........................ 1780

Cascio, Michael...... 1365, 1471, 1473

Casey, James .................................. 381

Casey, Laura................................... 1151

Cashen, Amanda ........................... 1496

Cason, Zelma ..................................... 547

Cassidy, Daniel P ............................ 90

Castells, Antoni .................. 625, 2139

Castillo, Paola ............................... 1573

Castle, Philip E.............................. 1208

Castonguay, Mathieu C.... 1994, 2009

Castro-Echeverry, Eduardo ........ 1367

Catenacci, Daniel ............................. 719

Cates, Justin MM .............................. 57

Catherino, William........................ 1121

Cathomas, Gieri ............................. 672

Cathro, Helen P........................... 2138

Catino, Anna Maria ..................... 1970

Catlow, Krista ................................ 122

Cattoretti, Giorgio .......................... 2136

Caturegli, Patrizio .......................... 1474

Caudle, Abigail S ............................. 472

Cavazza, Alberto .......................... 1905

Cayre, Anne............... 248, 779, 1107

Celis, Verónica .............................. 1409

Celli, Romulo...... 652, 653, 772, 820,

834,1776

Cellini, Ashley....................... 258, 259

Cenci, Tonia ..................................... 439

Centeno, Barbara............................ 603

Centonze, Giovanni............... 594, 595

Cerbelli, Bruna ................................ 1723

Cerda, Sandra ................................. 395

Cesari, Matthew ......1158, 1160, 1161,

Ch'ng, Sydney.............................. 1330

Cha, Eugene K ................... 850, 1058

Chadburn, Amy ................. 1464, 1465
Chadwick, Barbara E ..................... 405

Chadwick, Dianne ....................... 1835

Chaft, Jamie ................................. 2003

Chahine, Joeffrey ............... 1895, 2099

Chahine, Saad ..............................566

Chaib, Oumaima .... 1092, 1093, 1131

Chain, Krista ................................... 398

Chakrabarty, Bipasha .................... 762

Chakraborty, Baidarbhi ............ 3, 312

Chakravarthi, Balabhadrapatruni

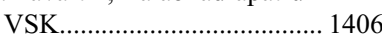

Chama, Allen C............................. 2025

Chambers, Setsuko........................ 1227

Chan, Alexander........................... 1368

Chan, Anna.................................. 1121

Chan, Cheri …………………............ 63

Chan, Emily ................ 348, 722, 1122

Chan, Joanna .................................. 1894

Chan, Kin .................................... 1070

Chan, May......................................... 497

Chan, Wilson................................. 1074

Chan, Wing C........ 1380, 1390, 1518 ,

1519

Chandan, Vishal S... 721, 1666, 1667, 1680

Chandarlapaty, Sarat .................. 1854

Chandler, Jocelyn B ....................... 349

Chandra, Ashish ................... 336, 411

Chang, Alicia H............................ 1564

Chang, Angela Y ............................. 316

Chang, Anthony ..... 1618, 1621, 1625

Chang, Chung-Che....................... 1365

Chang, Hong ......... 1372, 1432, 1487,

Chang, Jason C............................... 1903

Chang, Jenny................................... 164

Chang, Kai-Ping............................ 1579

Chang, Kenneth....................... 40, 1869

Chang, Koping ................................. 350

Chang, Martin C............................. 275

Chang, Michael ................................ 654

Chang, Nina ................................. 1904

Chang, Qing ..................................... 571

Chang, Ruby ..................................... 462

Chang, Steve ................................. 1340

Chang, Young Hwan ......................... 659

Chapel, David B .......................... 1123

Chapman, Christopher ................... 415

Chapman, Erin ................................ 1295

Chapman, Jennifer ............ 1369, 1431

Chapman, William C................... 1695

Charaghvandi, K Ramona............. 244

Charames, George........... 86, 88, 538,

Charles, Stoudenmire J ............. 1246

Charu, Vivek .... 124, 375, 1772, 2066

Charville, Gregory ..... 41, 1124, 1832

Chastain, Elizabeth .......... 1361, 1480

Chatterjee, Abhishek ...................... 214

Chatterjee, Deyali ................................ 1777

Chatterjee-Paer, Sudeshna........... 1204

Chau, Karen ………..................... 2077

Chau, Nicole G............................. 1310

Chaudhary, Adeel ............................ 627

Chaudhary, Rahman............. 175, 739

Chaudhry, Haroon .......................... 1919

Chauhan, Subhash............................... 1775

Chaux, Alcides .............................. 1019

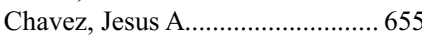

Cheah, Alison L................................ 31

Chebel Morello, Brigitte ................ 507 
Chebib, Ivan...................... 42, 62, 364 Cheeney, Gregory ........................ 1558 Chekmareva, Marina................... 1605 Chekol, Seble ................................ 1356 Chelliah, Adeline........................... 1905 Chelliserry, Jijumon ...................... 1056 Chen, Benjamin...... 1400, 1498, 1714 Chen, Betty ..................................... 572 Chen, Danqi ....................................... 725

Chen, Dong …………………..... 1397

Chen, Fei ....................................... 856

Chen, Guoli ................................... 1074

Chen, Hannah H........ 351, 586, 1704,

1826

Chen, Hui ....... 147, 1298, 1922, 2040

Chen, Huijiao ................................... 542

Chen, Jennifer .............................. 1472

Chen, Jie...................................... 1620

Chen, Jin .................................... 1851

Chen, Lee-may .............................. 1176

Chen, Li........................................ 1370

Chen, Min ......................................... 542

Chen, Mingyi ........................ 96, 1383

Chen, Rong .................................. 1371

Chen, Shaoxiong ........480, 852, 1010,

1021

Chen, Shaxioang .............................. 82

Chen, Sheau-Chiann .................... 1651

Chen, Shuo ................................... 2128

Chen, Tiffany .................................. 498

Chen, Wei.......... 655, 749, 796, 1091,

1696

Chen, Weina .......... 1355, 1378, 1484, 1508

Chen, Weiwei ....................... 125, 147

Chen, Weiyi.................................. 1427

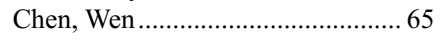

Chen, Wenjin................................. 1605

Chen, Xiaowei....................... 163, 571

Chen, Xin .................................... 1708

Chen, Yan .................................... 1372

Chen, Yao-Tseng .......................... 1334

Chen, Ye................ 1014, 2080, 2137

Chen, Yen-Ying ........................... 1724

Chen, Yi-Bin ………………….... 1381

Chen, Yi-Fan ................................. 2148

Chen, Yigu ................................... 2019

Chen, Yi-Hua ................................. 1464

Chen, Yingbei....................... 967, 1038

Chen, Ying-Bei.... 850, 874, 916, 935, 985, 1037, 1053, 1055, 1058

Chen, Yongmei.............................. 912

Chen, Yuan Yuan.... 1515, 1518, 1545

Chen, Yuhchyau .......................... 1907

Chen, Yunn-Yi..... 116, 171, 199, 200,

261,267

Chen, Zhengming........ 127, 128, 136,

$137,225,227$

Chen, Zi....... 1357, 1373, 1374, 1407, 1408,1423

Chen, Zongming E............. 656, 1682 Cheng, Angela S............... 1177, 1201

Cheng, Elaine Y ............................. 801

Cheng, Esther....... 126, 127, 128, 246

Cheng, Heather H........................ 1066

Cheng, Jason ................................... 1370

Cheng, Jason L.............................. 1906

Cheng, Jinjun .......................... 4, 1827

Cheng, Jinrong ...................... 875, 1082
Cheng, Liang..... 82, 561, 1003, 1004, $1021,1031,1033,1077,1552$,

1571,1736

Cheng, Lin.................................... 1661

Cheng, Mingyu ........................... 2118

Cheng, Tina ..................................... 1036

Cheng, Ting-Yuan David ............ 1907

Cheng, Xin Min ........................... 1840

Cheng, Yufan................................... 279

Cheng, Yu Wei................................ 424

Cheng, Yu-wei...................... 414, 519

Chennat, Jennifer S ............. 467, 1813

Chennubhotla, SC ........................ 1591

Cherian, Sindhu............................ 1399

Chernichenko, Maria.................... 1844

Chernock, Rebecca ........... 569, 1303,

1307,1346

Cherry, Athena ....... 1365, 1437, 1832

Chetty, Runjan.............. 639, 712, 802

Cheuk Leung, Hong ......................... 93

Cheung, Annie NY..................... 1262

Chevarie-Davis, Myriam............... 734

Chevez-Barrios, Patricia ............. 1729

Cheville, John C......... 888, 921, 922, 923, 931, 942, 967, 990, 1086

Chew, Jason B.

Chhieng, David ............... 406, 407, 485

Chi, Zhikai ...........................657, 658

Chianchiano, Peter ...................... 1688

Chiang, Sarah .......................1125, 1144

Chiappetta, Caterina......................... 66

Chibbar, Rajni .............................. 1105

Chibon, Frédéric .......................... 1128

Chic, Nuria .................................. 2139

Chiforeanu, Dan............................ 1444

Childs, Margaret........................... 1875

Chinegwundoh, Francis ................ 858

Chinnaiyan, Arul M .... 123, 497, 590, $1049,1050,1069$

Chinnaswamy, Girish ...................... 80

Chiosea, Simion I.............................. 600

Chiosea, Simon ....... 551, 1282, 1294,

1300,2122

Chioureas, Dimitrios ........ 1354, 1492

Chiriboga, Luis ............................ 1969

Chirieac, Lucian R ....................... 1931

Chirip, Venus.................................. 1793

Chisholm, Karen M........... 1365, 1472

Chitale, Dhananjay...... 156, 157, 221,

2043

Chiu, April........................ 1375, 1494

Chkheidze, Rati........................... 1725

Chlipala, Elizabeth............................... 2026

Chng, Tze Wei....................... 129, 198

Chng, Wee-Joo ................................ 1468

Cho, Byoung Chul ...................... 1296

Cho, Eun Yoon ....................... 130, 131

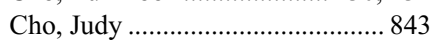

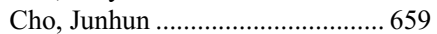

Cho, Margaret ............... 343, 352, 647

Cho, Woo Cheal ............................... 660

Cho, Yoon Ah............................... 1296

Choi, Euna......................................... 353

Choi, Eun-Young K......................... 726

Choi, Hyejeong ................. 1778, 1799

Choi, Julia ..................................... 1367

Choi, Kyung Un ...................... 724, 771

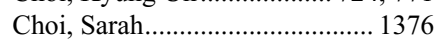

Choi, Sung-Eun............................. 1635

Choi, Won-Tak ... 661, 662, 817, 1673

Choi, Yoonla.

60
Choksi, Rachana ...................... 5, 312

Chon, W James ............................. 1625

Chopra, Amit............................... 1919

Chopra, Shefali ........................................... 67

Chosdol, Kunzang......................... 1335

Chottová-Dvořáková,

Magdalena......

Chou, Pauline $\quad 1877$

Choueiri, Toni K ................................. 949

Cho-Vega, Jeong Hee................... 1431

Chow, Christine.................. 1201, 1787

Choy, Bonnie................. 354, 876, 877

Chren, Mary-Margaret .................... 515

Christensen, Hannah M................ 1908

Christensen, Paul........................... 370

Chu, Jinah .......... 130, 131, 633, 1778

Chu, Kevin ................................... 2026

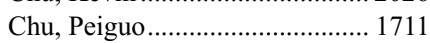

Chu, Tianming................................. 267

Chu, Ying-Hsia............. 573, 574, 602

Chua, Kenon S .................................. 63

Chuang, Shih-Sung ...................... 1468

Chui, Michael Herman................. 1126

Chumbalkar, Vaibhav ..................... 132

Chung, Ji Myung.............................. 1296

Chung, Jin-Haeng ....................... 1993

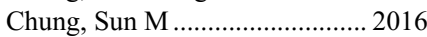

Chung, Yumin ...................... 254, 255

Church, James .................................. 706

Churg, Andrew ................................... 1946

Chute, Deborah ........... 543, 604, 1322

Cibas, Edmund S................... 607, 614

Cicek, Ali .................... 294, 299, 308

Cicek, Ali Fuat ................................ 133

Cieply, Kathleen............................ 1034

Cimbaluk, David ........................... 1616

Cimic, Adela ..................... 1581, 1883

Cimická, Jana............................... 1178

Cimino-Mathews, Ashley ... 124, 266,

$281,282,297$

Ciomek, Natalie A....................... 1377

Citron, D .................................... 1306

Clark, Beth Z..... 141, 182, 209, 1103 ,

1258,2103

Clark, Ian........................ 1662, 1709

Clark, Jonathan ....... 1305, 1330, 1333

Clark, Peter E ............................... 1083

Clarke, Ann E............................... 1526

Clarke, Blaise ..........1126, 1137, 1184,

$1185,1251,1275$

Clarke, Loren ................................ 518

Clay, Michael R ........................... 1890

Clayton, Adam ................................... 1477

Clemens, Mark W ........................ 1428

Clement, Philip B ........................... 1250

Clements, June ............................. 2114

Clemmensen, Tyler ........................ 878

Clendenning, Mark ............... 791, 792

Clerc, Johanna........................ 507, 508

Climent, Fina.................................... 1475

Clinton, Lani K ............ 414, 663, 664

Cloe, Adam ................................. 1370

Clouston, David ............................. 1065

Cluxton, Chris .............................. 1828

Clymer, Beth ................................. 2124

Coach, Julia.................................... 122

Coates, Ryan ...................................... 665, 666

Cobb, Jared ................................... 1849

Coberly, Jared ………........................ 1378

Cobos Sillero, Maria ......................... 22

Cocchi, Stefania ............................... 47
Cochrane, Dawn R ....................... 1201

Cocker, Rubina S ....................... 2077

Cocks, Margaret........................... 1127

Coen, Matteo ..................................... 321

Coffey, Amy M ........................... 1379

Coffman, Brittany B.......................... 25

Cogdell, David E............................. 883

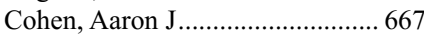

Cohen, Arthur H.......................... 1651

Cohen, Cynthia ... 104, 177, 216, 217,

$362,365,479,632,837,932$, $1228,1299,1336,1575,1657$,

1737, 1964, 2110, 2143, 2146

Cohen, David W.......................... 1309

Cohen, Jarish.................... 1663, 1829

Cohen, Jean-Marc .................. 344, 447

Cohen, Mark …………...... 1740, 1756

Cohen, Paul J ................................. 1481

Cohen, Victor ................................ 2005

Colafrancesco, Serena................. 1723

Colasse, Elodie............................. 1973

Colby, Thomas V .......................... 2009

Cole, Gary ......................................... 410

Colecchia, Maurizio ......................... 879

Colling, Richard.....................260, 712

Collins, Brian ................................. 342

Collins, Jennifer A............................ 880

Collins, Lucine ............................ 2158

Collins, Michael T.......................... 761

Collins, Scott A ................................ 2135

Collinsworth, Amy .......................... 654

Colomo, Luis..................... 1348, 1475

Comperat, Eva.......... 881, 1008, 1070

Compton, Margaret .......................... 28

Conceição, Raquel C...................... 260

Conces, Miriam R ......................... 1888

Cone, Molly ..................................... 687

Connelly, Caitlin ...... 994, 1005, 1924

Connors, Katherine ...................... 1874

Conrad, Douglas …………………... 727

Contreras, Alejandro ...................... 114

Conyers, Jeffrey A.............................. 27

Cook, James R .................. 1415, 1499

Cooney, Anthony........................... 1860

Cooper, Benjamin ........................ 1867

Cooper, Dennis................................ 1359

Cooper, Kumarasen...................... 1200

Cooper, Lee AD .............................. 1760

Copeland, Karen ............................ 2026

Copeland Jr, Robert L ................... 193

Coppa, Jorgelina....................... 594, 595

Coppola, Domenico …….... 750, 2105

Corazza, Gino R.............................. 819

Corcoran, David L........................... 244

Corey, Eva ....................................... 2113

Coric, Marijana ............................... 984

Corliss, Robert ................................ 556

Cornejo, Kristine....... 461, 1052, 1167

Cornell, Lynn D .... 1615, 1619, 1626,

1652

Cornish, Toby C .......................... 2113

Correa, Hernan................................ 2082

Corredor, German ............. 1607, 1864

Corse, Tanner D ............................. 1328

Cortese, Cherise ............................ 1232

Corti, Barbara............................... 1199

Cortright, Valerie....................... 665, 666, 2034

Corvalan, Alejandro H ................ 1533

Cosar, Ediz F ....................... 461, 1811

Cosio, Fernando ................ 1619, 1652

Costa, Estela 159 
Costa, Larissa BE......... 862, 863, 864 Costello, Brian A.......................... 922 Costinean, Stefan ......................... 1380 Cote, Michele L.......................... 113, 139, 175 Cote, Richard J.............................. 195 Cotelingam, James .......................... 2105 Cotler, Scott ................................. 1704 Cotta, Claudiu V ............................ 759 Cotter, Maura ................................. 120 Cottrell, Tricia R ................... 43, 1706 Cottrill, Elizabeth............................. 613 Cotzia, Paolo .................................... 668 Couce, Marta E …….......... 1740, 1756 Coughlin, Geoff ........... 884, 885, 886 Courville, Elizabeth L..... 1502, 1522,

1547

Cousineau, Craig .............. 1664, 2027

Couto, Joe ..................................... 1039

Covach, Adam................................ 575

Covinsky, Micheal .......................... 27

Cox, Bettye ................................... 328

Cox, Brian .................................... 1559

Cox, Jesse......................... 1197, 1582

Cox, Roni ......................... 1001, 1012

Coy, Shannon ................................. 1726

Cracolici, Vincent M........ 1618, 1621

Craddock, Kenneth J.................... 1432

Crafter, Sharon .............................. 2151

Craig, Jeffrey W .......................... 1509

Craig-Owens, Laura ........................ 548

Cramer, Harvey ...................... 353, 442

Cramer, Harvey M ............................ 24

Crane, Genevieve M ..................... 551

Crapanzano, John .......................... 1928,1965

Crawford, John............................. 1880

Crawford, Judith A....................... 1985

Crawford, Scott ............................ 2026

Crawford, Troy............................... 2108

Crespo, Gonzalo............................ 1685

Crisi, Giovanna M........................ 1841

Crist, Henry................ 612, 613, 1342

Cristando, Camilla ................ 134, 135

Croce, Sabrina ............................... 1128

Cronin, Mary Kate ....................... 1701

Cross, Janet ..................................... 146

Crotty, Rory ....................................... 1381, 2028

Crowe, Sheila E .......................... 1559

Crowe, William ............................... 2120

Cruise, Michael ...................... 705, 706

Crum, Christopher P ........1162, 1163, $1210,1212,1242,1243,1931$

Crumley, Suzanne ............1129, 1130,

$1148,1149,1830$

Cruz, Ofelia................................... 1893

Cuatrecasas, Miriam .......... 625, 2139

Cubilla, Antonio.... 1017, 1027, 1028,

1029

Cuda, Jackie .................................. 356

Cuervo, Juliana ............................. 1574

Cuevas, Martin...... 1017, 1027, 1028,

1029

Cuevas-Ocampo, Areli K..... 73, 1727

Cui, Isabelle H ..................... 576, 669

Cui, Min .................................... 1779

Cui, Wei............................ 1364, 1495

Cui, Xiaoyan ........ 136, 137, 225, 227

Cui, Ya....................................... 2029

Cullen, Jennifer .......... 871, 912, 1076

Culver, Brady P................. 2119, 2121

Cummings, Oscar W ...................... 726
Cunha, Isabela W ............ 1018, 1027, 1028, 1029, 1059

Cunningham, Ashley M ............. 1382

Cunningham, Vickie L ................. 2059

Curci, Nicole....................... 909, 1068

Curry, Choladda V ....................... 1379

Curry, Jonathan L........ 525, 533, 537, $1379,1758,2018$

Curtis, Mark T.................. 1733, 1741

Cuzick, Jack ................................... 861

Cymes, Karina............................... 1773

Cyrta, Joanna....................... 882, 1857

Czader, Magdalena.............. 1466, 1523

Czapla, Agata ............................ 2030

Czauderna, Piotr......................... 1875

Czeczok, Thomas.. 1615, 1665, 1666,

1667,1687

Czerniak, Bogdan....... 883, 933, 1071

D'Agati, Vivette ........................... 1651

D'Alfonso, Timothy.... 126, 127, 128 ,

$\begin{array}{rr} & 246 \\ \text { d'Amati, Giulia } & 1723\end{array}$

Daaboul, MHD Fayez ........... 113, 139, $175,1092,1131$

Dabbs, David J.... 140, 141, 182, 209 , $1103,1174,1258,2103$

Daber, Robert D ................ 1504, 1510

Dacic, Sanja ............. 642, 1951, 2000

Dadhania, Vipulkumar ................... 883

Dagà, Adrià L................................... 355

Dagher, Julien .............. 884, 885, 886

Dahiya, Sonika ............................ 1726

Dahl, Gary .................................... 1506

Dahly, Darren............................... 1905

Dai, Ding...

1909

Dairiki, Mutsumi.......................... 1909

Dalbagni, Guido...... 985, 1037, 1055,

1058

Dal Cin, Paola .......... 950, 1321, 1931

Dallol, Ashraf.................................. 627

Dalton, Leslie........................ 142, 143

Dalvi, Siddhartha ............ 1297, 1391,

1392, 1919

da Matta, Francisca IBCC............. 138

Damodaran, Chendil ................... 1858

Damodaran, Senthil............................ 147

Danakas, Alexandra ............. 870, 887

Danel, Claire ................................. 1950

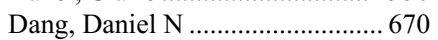

Daniels, Molly................................1114

Danner, Derek ....................... 413, 1203

Dansereau, Francois....................... 550

Dansette, Delphine......................... 646

Dantey, Kossivi ..................... 356, 357

Darcy, Kathleen M ........................ 1261

Darga, Elizabeth P.......................... 123

Darke, Amy K .............................. 1907

Darras, Natasha ............................. 358

Darvishian, Farbod...... 305, 306, 307,

1592

Das, Binita....................................... 2124

Das, Kasturi..................................... 2077

Dasaraju, Sandhya............... 437, 2085

Dashti, Nooshin K.................. 44, 888

Da Silva, Annacarolina .......1110, 1803

Dastani, Zari.................................. 2005

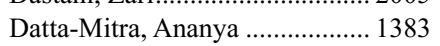

Datto, Michael................................. 389

Daugaard, Søren.............................. 517

Daum, Ondrej.................... 7, 849, 983

Dauplat, Marie-Melanie ................ 248
Davenport, Matthew .......... 909, 1068

Davey, Scott .................................. 643

Davicioni, Elai ......... 930, 1056, 1085

Davick, Jonathan J ........................ 889

David, Marjorie P......................... 2031

David, Stephanie N .... 144, 251, 1246

Davidoff, Andrew M.................. 1890

Davidson, Tara M........................ 2032

Davies, Michael A................. 533, 537

Davila, Jaime.......................... 74, 689

Davion, Simone............................. 1384

Davis, Drew ........................... 558, 671

Davis, Eric M ............................... 2119

Davis, John W ............................. 1064

Davis, Thaylon ............................... 518

Davis, Warren................................. 1907

Davison, Jon......................... 642, 2033

Dawson, Heather.................... 672, 673

DCruz, Anil .................................. 1289

de Abreu, Francine B ........... 523, 769,

1960, 2129

de Alava, Enrique.......................... 1870

Dean, Christina ................................ 558

Dean, Stephanie ............................. 319

de Andrade, Mariza ....................... 1956

Deaton, Ryan................... 2148, 2150

Deavers, Michael .............. 1912, 1913

Debelenko, Larisa ............. 1025, 1871

Debeljak, Marija .............................. 761

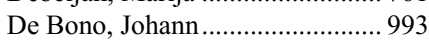

De Brot, Marina ............................. 138

Decamp, Malcolm......................... 1906

Decker, Melissa.............................. 292

de Cotiis, Dan.................................... 1894

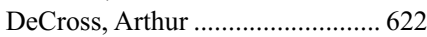

Dedes, Konstantin .......................... 291

Deeken-Draisey, Audrey ..... 577, 674,

Deen, Malik................................ 1605

de Figueiredo, Carlos H ................. 138

de Freitas, Declan........................... 1614

Degnim, Amy ................................... 264

DeGraff, David J .......................... 1074

deGuzman, Jose ........................... 2099

Deharvengt, Sophie J .......... 523, 769,

2129

Dehner, Louis P...

De Iaco, Pierandrea

....... 2

Deisch, Jeremy

1199

Dei Tos, Angelo P $\quad 46,47,81,83$

De Kock, Leanne ........................ 1251

Delahunt, Brett.... 884, 885, 886, 895,

896

Delair, Deborah...... 1144, 1234, 1910

de la Oliva, Jorge ................ 359, 1348

Del Conte, Gabriele ....................... 594

De Leo, Antonio................ 1199, 1780

de Leval, Laurence......................... 1363

Delgado, Silvia................................. 159

dell'Aquila, Marie.............................. 1754

Della Rocca, Carlo ............................. 66

Del Portillo, Armando ........... 675, 840

del Pozo-Yauner, Luis .................. 1630

Delprado, Warick .......................... 1603

DeLuca, Juliana.................................... 402

De Luca-Johnson, Javier.............. 2034

Demaria, Sandra.......................... 127, 128

De Marzo, Angelo M ........... 124, 853, $860,993,1056,2113$

Demetri, George............................ 1847

Demetris, Anthony J..

1847
Demicco, Elizabeth G ............ 45, 500

Deng, April....................................... 503

Deng, Fang-Ming ........ 571, 856, 920 , 1000, 1072, 1073, 1089, 1090,

1592

Deng, Xiaobing.....

.. 529

Dennis, Katie.......................... 189, 431

Denroche, Rob E......................... 1835

Deodhar, Kedar ................................... 1797

De Palma, Angela........................ 1970

de Peralta-Venturina, Mariza ...... 892,

1387

de Ridder, Gustaaf........................ 1701

Derkyi-Kwarteng, Leonard ......... 1097

Desai, Neil........................ 1055, 1058

Deshpande, Charuhas................... 2063

Deshpande, Vikram......... 42, 62, 118,

$631,708,765,1266,1362,1679$,

2028

Desmeules, Patrice........... 1831, 1910

1911

Desouki, Mohamed M ........ 144, 251,

$1132,1142,1246$

Dessanti, Paolo............................... 1923

Dessen, Philippe............................... 473

De Stefano, Danielle ...................... 502

D Estopinal, Maria ........................ 1728

de Torres, Inés ................................. 890

Devereaux, Kelly ............... 676, 1832

De Villa, Vanessa Grace M ........ 2035

Devitt, Katherine A ....................... 1413

Devouassoux-Shisheboran,

Mojgan ................................. 1128

Dewar, Rajan........... 549, 1376, 1541 ,

1597,2046

Dey, Vivek .........................................516

DeYoung, Barry R......................... 2092

Dhall, Deepti .............. 637, 830, 1773

Dhanasekara, Saravana ................. 497

Dhanasekaran, Saravana ............. 1050

Dhand, Abhay ……………........... 1565

Dhani, Neesha .................... 1185, 1835

Dhillon, Jasreman .................. 393, 986

Dhingra, Sadhna.......................... 1668

Dhir, Rajiv........................ 1006, 1034

Dhorajiya, Poojaben...................... 1345

Dialani, Vandana ................................. 284

Dias, Beatriz.................................. 1768

Dias-Santagata, Dora ........ 1294, 1963

Díaz, Alba .................................. 1685

Diaz, Sherley....................................... 1983

Diaz-Cano, Salvador ........... 578, 1833

Diaz-Gomez, Berlly L......... 360, 1205

Dickler, Maura ….................. 285, 286

Dickson, Brendan C ............ 56, 86, 88 ,

538,2151

Di Cristofano, Claudio ...................... 66

Didonato, Rosemarie....................... 145

Diez-Valle, Ricardo...................... 1739

Dill, Erik .......................................... 146

Dillon, Deborah A............... 202, 1809

Dillon, Jessica ..........1133, 1134, 2037

Dillon, Patrick .................................. 164

Di Loreto, Christina ...................... 1749

DiMarco, Michelle........................... 1622

Di Meo, Ashley ................................. 891

Dimichelis, Francesca........................ 1853

Dimitravo, Nevenka...................... 1565

Dimitrova, Nevenka.......... 1025, 1646

DiNardo, Courtney....................... 1434

Ding, Qingqing ..................... 125, 147 
Ding, Xiangdong ........................... 1269 Ding, Xianzhong ... 1600, 1704, 1826, 2048

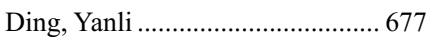

Ding, Yi.......................... 1385, 2120

Dinney, Colin .................................... 883

Diorio, Caroline ........................... 1962

Dirks, Dawn C ............................ 1209

Di Sabatino, Antonio...................... 819

Dittmann, David.................. 711, 1842

Divatia, Mukul ...................... 36, 1729

Divya, P........................................ 648

Djalilvand, Azita ………………... 2114

Djordjevic, Bojana .......... 1093, 1158, $1159,1160,1161,1218$

Dlouhy, Ivan................................. 1409

Dmetrichuk, Jennifer M..... 760, 1580

Dmitriev, Alexey ........................... 1845

Dodd, Anna J.............................. 1835

Dodd, Leslie …………………….... 379

Dogan, Ahmet ............................ 1368

Dogan, Snjezana ............... 1298, 1837

Dolderer, Daniel.......................... 1135

Dolezal, Anna................................. 1386

Dolkar, Tsetan .............................. 1136

Domingo-Vidal, Marina .............. 1952

Dominguez, Carolina ....... 1560, 2038

Domínguez, Emeli ....................... 1101

Dominguez, Hugo ......................... 1220

Dominguez Marquez, Carolina.... 815

Dominika, Ryba .......................... 1600

Don, Michelle .......... 892, 1387, 1623

Donaldson, Alana R ............. 148, 149

Donati, Davide M............................. 47

Done, Susan J............................. 2035

Dong, Fei........833, 1162, 1163, 1212, 1931, 1934, 1977

Dong, Yimin ...................................... 678

Donson, Andrew .......................... 1743

Dorfman, David M....................... 1528

Dorko, Ken................................... 1686

Dorn, David...................................... 893

Dorris, Kathleen ........................... 1743

Douds, Jonathan J ....................... 1388

Dowdy, Sean C............................ 1131

Dowling, Ryan JO........................... 275

Downes, Michelle ........................... 867

Downes, Michelle R...................... 1008

Downing, James R ...................... 1890

Doxtader, Erika E.......................... 361

Doyle, Alden ................................. 1617

Doyle, Brendan ........................... 1614

Doyle, Leona................................... 1809

Doyle, Maria M............................. 1660

Doyle, Scott........................ 1341, 1345

Draganova-Tacheva, Rossitza...... 381

Drage, Michael G........................... 804

Drakos, Elias ..................... 1354, 1492

Dresser, Karen......... 704, 1167, 1400,

1498

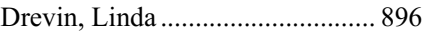

Drilon, Alexander.............. 1837, 1953

Driver, Brandon ............... 1912, 1913

Dromparis, Peter .......................... 1914

Drotar, Nancy ........................................ 1

Drui, Delphine.................................. 601

Drumheller, Bradley...................... 1299

Drwiega, Joseph ............................ 1406

Dry, Sarah ....................... 2039, 2054

$\mathrm{Du}$, Jing ......................................... 150

Duan, Kai ........................... 579, 1137 du Bois, Andreas ............................ 1201

Dubova, Magdalena ........................... 7

Duboy, Jon ................................... 1588

Dubuc, Adrian ................... 1720, 1745

Ducar, Matthew ............................. 1803

Duensing, Stefan ............................... 996

Duffield, Amy .............................. 1555

Dugay, Frédéric.................... 946, 948

Duggan, Maire .......................1183, 1191

Duke, Linnea.................................... 550

Duker, Nahum ................................ 3, 5

Dulau Florea, Alina ...................... 1389

Dummer, Reinhard.......................... 495

Dumouchel, Justin......................... 1947

Dumur, Catherine I ............ 433, 1976

Duncavage, Eric..... 498, 1190, 1350,

1496

Dunglison, Nigel.......... 884, 885, 886

Dunne, Richard F ......................... 1803

Dunseth, Craig ............................. 1781

Dunya, Gabriel............................ 1872

Duose, Dzifa .................................. 513

Dupont, Andrew W ....................... 630

Dupuis, Frantz.............. 946, 947, 948

Dupuy, Damian E......................... 2135

Dureau, Zachary ................... 362, 894

Durkin, Lisa ................................... 1414

Dursun, Nevra ............................ 1784

Duska, Linda ................................ 1276

Dvanajscak, Zeljko .......... 1559, 1561,

1915

Dzinic, Sijana ................................. 740

Early, Caroline .................................... 8

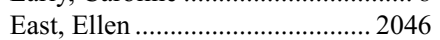

East, Ellen G ................................ 151

Eastham, James ............................... 935

Ebb, David ......................................... 22

Eberlein, Jens ............................... 2121

Ebert, Benjamin ............... 1542, 1543

Eble, John N...... 23, 561, 1003, 1004, 1031, 1033, 2133

Edeiken-Monroe, Beth S............... 456

Edeline, Julien............. 947, 948, 1805

Edelweiss, Marcia ................. 242, 243

Eder, Matthias .............................. 1945

Edgerton, Mary E.................................... 2025

Edlefsen, Kerstin L ...................... 1363

Edmund, Liz................................ 1916, 1917

Edupuganti, Subhash.................... 1548

Edwards, David........................... 1849

Edwards, Dean P.............................. 114

Edwards, William D....................... 309

Efstathiou, Eleni.................................... 1064

Egan, Caoimhe ............................. 1390

Egevad, Lars ....... 846, 884, 885, 886, $895,896,1066$

Eghtesad, Mansooreh........... 288, 289

Ehdaivand, Shahrzad...................... 176

Eich, Marie-Lisa .... 1018, 1019, 1060

Eickhoff, Jens.............................. 1479

Eifert, Cheryl L ............................ 2129

Eisch, Robin................................ 1536

Eisenhut, Carol.............................. 1121

Ekladous, John ............................... 475

Elalfy, Zahraa S.............................. 945

Elbanan, Mohamed G ................... 938

El Dinali, Mohamed..................... 1361, 1412

Elemento, Olivier... 1463, 1853, 1857

Elenitsas, Rosalie ........................ 1493

Elfandy, Habiba............................... 897

Elfatairy, Kareem .................. 910, 911
El-Gendi, Saba ...................... 152, 898

Elghetany, Mohamed T .............. 1379

El Hag, Mohamed ........................ 1236

E1 Hajj, Hiba .................................. 1567

El Hallani, Soufiane ........ 1300, 1730,

2122

ElHassan, Hiba.............................. 1833

El Hussein, Siba .................. 303, 1918

Eliaszadeh, Shohreh................... 1179

Elishaev, Esther................ 1103, 1258

E1 Jabbour, Tony .... 1391, 1392, 1919

El Jamal, Siraj M....... 847, 917, 1361, 1393, 1412, 1480, 1718, 1882,

1889,2024

Ell, Christian 679

Ellenson, Lora ................................ 1204

Elli, Francesca M ............................ 47

Ellimoottil, Chandy............. 909, 1068

Elliott, Bruce .................................. 257

Elliott, Robin.................................. 1603

Elliott Range, Danielle.................. 580

Ellis, Carla.................. 313, 341, 1648

Ellis, Ian ........................................... 252

Ellison, David .............................. 1890

El-Mofty, Samir K ........... 1282, 1307,

Elmore, Joann G.......................... 2032

El-Naggar, Adel K......................... 473

Elortza, Felix................................. 1875

El-Rayes, Bassel .............................. 787

El-Rayes, Dina .............................. 991

Elsayes, Khaled M ......................... 938

Else, Tobias .................................... 590

Elshamy, Mohammed .................. 1655

Elsheikh, Tarik M......................... 408

Eltoum, Isam-Eldin ............ 470, 1313

Elvin, Julia A....... 196, 238, 496, 790, 994, 1005, 1015, 1138, 1297, $1331,1654,1836,1855,1880$, 1920,1924

Elwahab, Sami ............................... 120

Ely, Kim .............................. 477, 1303

El-Zammar, Ola ........................... 1955

Emberesh, Myesa .............. 1412, 1480

Emens, Leisha A.................. 281, 282

Emerson, Ryan ................................. 1493

Emile, Jean-Francois...................... 779

Emmadi, Rajyasree ........................ 1179

Enbom, Elena .................................... 363

Eng, George ………....................... 364

Engelman, Donald M.................... 2108

England, Jonathan S...................... 438

English, Dallas ………………….... 792

Enke, Charles A.............................. 1527

Enlow, William ................................. 1925

Ennis, Marguerite............................ 275

Ennis, Rachel ................................ 120

Epari, Sridhar ................................... 1762

Epelbaum, Fanya........................... 2077

Epstein, Jonathan I ....... 865, 866, 880, $899,904,913,928,953,956$, $961,1011,1013,1079,1084$,

Erbarut, Ipek $\quad 2133$

Erdem Toslak, Iclal .......................... 586

Erho, Nicholas G...... 930, 1056, 1085

Erickson, Lori ...................... 535, 900

Erlich, Annette ............................. 1070

Ernst, Wayne ............................... 1006

Ertan, Atilla ......................... 630, 1819

Ertoy, Dilek.

1018
Eschbacher, Jennifer ...

1760

Eschrich, Steven A ......................... 750

Esebua, Magda ... 482, 901, 958, 2053

Eshleman, James R ............ 761, 1555

Eskandari, Ghazaleh ................... 1668

Eskandari, Mohammad ................ 1394

Eslami, Arash ............................... 1921

Esmaeili Shandiz, Alaleh ........... 902,

1063,1395

Esmaeli, Bita ................................ 1758

Esnakula, Ashwini K................. 654, 726 ,

1674,1675

Esopi, David.......................................... 853

Esper, Lawrence ........................... 2144

Espinal, Paula S ............... 1030, 1328

Espinal, Paula Sofia ....................... 195

Esposito, Evangelina........ 1731, 1767,

1768

Esposito, Irene............................... 1773

Esposito, Michael J ......................... 563

Esteller, Manel ............................... 757

Estrada, Monica V......................... 164

Estrine, Dolores............................. 1461

Eszlinger, Markus ........................... 607

Ettel, Mark ...................................... 856

Ettler, Helen ..............1153, 1154, 2042

Euscher, Elizabeth...1093, 1139, 1140

Evans, Andrew ........ 773, 1385, 1430,

1439

Evans, Andrew J................. 891, 1008

Evans, Chris .................................... 934

Evans, James J .............................. 1741

Evans, Mark ..................................1115

Evasovich, Maria ……........ 450, 2085

Ewalt, Mark D................................... 734

Ewaz, Abdulwahab ........................ 365

Ewton, April ...................................... 1729

Ewunonu, Henry ............................. 767

Eze, Ogechukwu Pearl....... 679, 680, $780,781,798,811,812,825$

Fabrizio, David .......... 496, 790, 994, $1005,1015,1138,1297,1331$, 1654, 1836, 1855, 1920, 1924

Fadare, Oluwole.......... 172, 173, 174, $1132,1141,1142,1143,1164$, $1165,1224,2140$

Fadda, Guido............... 336, 411, 490

Fakhry, Carole ............................... 1302

Fallon, John T ......... 924, 1023, 1025, $1274,1563,1565,1646,1765$, $1820,1871,2147$

Falzarano, Sara M ……………...... 903

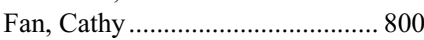

Fan, Fang........................ 189, 190, 431

Fan, Hongxin..................................... 499

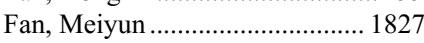

Fan, Minghua ................................ 1088

Fan, Rong...................................... 1021

Fan, Wen …........................ 2061, 2062

Fan, Xuemo................. 554, 572, 1593

Fanburg-Smith, Julie........ 1562, 1873

Fang, Catherine …................. 827, 828

Fang, Hong...................... 1396, 1397

Fang, Lianghua ................. 1922, 2040

Fang, Xiangming................................ 564

Fang, Yanan.................................... 2074

Faquin, William.......................... $281,582,1294$

Faquin, William C .......................... 358

Farag, Mina …………………....... 1024

Faragalla, Hala ..................................... 691

Farahani, Navid.............................. 734 
Farghaly, Hanan ............................... 681

Farhat, Nada .................................. 581

Farid, Heba.................................... 898

Farinha, Pedro ............................... 1526

Farmer, James .............................. 1722

Farooq, Fahad ............................... 1449

Farooq, Taliya ......... 904, 1563, 1697,

1732,1866

Farooqi, Midhat............................ 1493

Farrag, AbdelRazik H .................. 945

Farrell, Jessica M ............................. 366

Farris, Alton B.............................. 1648

Farshid, Gelareh.............................. 153

Faruqi, Asma ................................. 1151

Farver, Carol …................... 543, 1955

Fasanella, Kenneth E ......... 467, 1813

Fathallah, Lamia ......................... 1094

Fatheree, Lisa A .................. 2068, 2069

Fatima, Nazneen ........................ 2123

Faulkner-Jones, Beverly E........ 1584,

1624,1649

Fayad, Miriam............................... 514

Fayed, Doha E............................ 1751

Fayyaz, Ahmed U........................... 309

Fayyaz, Silvat S .............................. 168

Fazeli, Roghayeh............................ 390

Fazlollahi, Ladan.......................... 1928

Feasel, Patrick …............................. 500

Fedeli, Franco ….......................... 1923

Federman, Noah C ...................... 1713

Fedor, Helen.................................. 2113

Fedorova, Maria ............................ 1844

Feely, Michael....................... 654, 775

Feeney, Margaret.......................... 2059

Fehniger, Todd ………………..... 1496

Fei, Baowei ..................................... 998

Feilotter, Harriet................................ 643

Feldman, Alexander ...................... 905

Feldman, Andrew ......................... 1512

Feldman, Darren ............................ 874

Feldman, Michael ........... 1583, 1603,

2141

Felisiak-Golabek, Anna........ 501, 702

Felix, Ashley .............................. 1192

Felix, Juan ....................................... 363

Fels Elliott, Daffolyn R................ 1834

Fend, Falko ….............................. 1507

Feng, Xiaojun.................... 1823, 1852

Feng, Yingdong................... 875, 1082

Fennen, Erika ................................. 565

Feratovic, Rusmir.......................... 404

Ferda, Jiri .......................................... 7

Fernandes, Helen..... 697, 1823, 1848,

1852,1937

Fernandez, Anthony P ................... 530

Fernandez, Martin P..................... 510

Fernandez, Pedro............................ 154

Fernandez, Susan ................. 330, 331

Fernandez-Delgado, Rafael........ 1409

Fernandez-Pineda, Israel............. 1890

Fernandez-Pol, Sebastian... 502, 1398

Ferrarrotto, Renata ...................... 1290

Ferrazza, Laura ............................... 223

Ferré, Elise MN............................... 626

Ferreccio, Catterina......................... 1774

Ferreira, Joana ................................ 367

Ferrell, Linda.......... 1676, 1677, 2133

Ferrell, Paul B …………………... 1510

Ferrell, PB .................................... 1504

Ferrer, Alejandro ........................... 1467

Ferro, Paola ................................... 1923
Ferrone, Cristina ............................. 631

Ferrone, Soldano ............................. 631

Ferry, Judith A................... 1362, 1363

Fervenza, Fernando C ..... 1641, 1642,

$\begin{array}{lr} & 1643 \\ \text { Fetais, Alanoud R } & 1560\end{array}$

Ffrench, Brendan........................... 1860

Fidai, Shiraz ........................ 906, 907

Fidan-Ozbilgin, Ozlem ................. 333

Fidler, Mary E ................... 1619, 1626

Fiel, M Isabel ......... 1689, 1690, 1691

Fields, Kristina .............................. 1049

Fierro-Fine, Amelia............. 553, 1301,

2023, 2088

Filardi, Dominic A …..................... 293

Filippo, De Braud............................. 595

Finch, C....................................... 1306

Findeis-Hosey, Jennifer J .... 622, 682, $683,699,1669,1702,1803$

Fine, Jeffrey L ............................. 1591

Fine, Samson W ................. 850, 874, $916,935,985,1037,1038,1053$,

1055,1058

Fineberg, Susan ............................... 145

Fink, Daniel...................................... 291

Finkelstein, Sydney........... 339, 1285,

1822,2111

Fiscella, Julietta............................ 2095

Fischer, Andrew ................... 446, 451

Fischer, Andrew H ........................... 464

Fischer, Sandra .............................. 1835

Fish, Jennifer................................... 257

Fishbein, Gregory A...................... 314

Fisher, Hugh A ........ 994, 1005, 1015,

$\begin{array}{lr} & 1654 \\ \text { Fisher, Kurt } & 2124\end{array}$

Fitzgerald, Michael A.................... 1589

Fitzgerald, Rebecca C ................. 1834

Fitzgibbons, Patrick L...... 2068, 2069

Fix, Daniel................................... 1144

Flake, Darl...................................... 518

Flannelly, Grainne......................... 435

Flavin, Ricahrd.............................. 1860

Fleming, Evelyn L........................ 1134

Fleming, Jason B.............. 1777, 1785

Fleming, Jude .................................. 723

Fleshner, Neil E............................ 1070

Fletcher, Christopher DM ....... 33, 51, $52,68,84,1910$

Fletcher, Jonathan ............................ 1847

Flood, Trevor A .............................. 1048

Flotte, Thomas J................. 535, 2084

Flynn, Jefferey ……........................ 292

Fogo, Agnes ........... 1629, 1633, 1651

Fogt, Franz ……………............... 2157

Foley, Carolyn............................. 1473

Folkerth, Rebecca ............ 1746, 1757

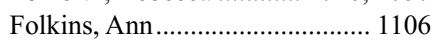

Foll, Matthieu................................ 1821

Follett, Robert ....................................... 1612

Folpe, Andrew L ... 31, 37, 44, 74, 75,

$689,931,1999$

Fong, Andrew.............................. 1564

Fonseca, Isabel ............................. 1300

Fonseca, Pedro ............................... 1492

Fonseca, Ricardo ............................ 367

Fonstad, Rachel.............................. 2114

Foo, Wai Chin .......... 684, 1777, 1785

Foran, David ............................... 1605

Forcada, Pilar ................................ 1475

Forcione, David G......................... 438
Forcucci, Jessica A......................... 273

Ford, Andrew ............................... 1313

Forde, Patrick M ………………... 2003

Foreman, Nicholas ............................ 1743

Forero, Andres................................. 164

Fornino, Daniele ............................... 819

Forns, Xavier................................. 1685

Forrest-Hay, Alex ......................... 1034

Forse, Catherine .............................. 155

Forster, Robert …......................... 2132

Fort, Victoria ......

1195

Fortuna, Danielle.................. 685, 1733

Foster, Nathan R ............................. 722

Foulke, Llewellyn ........................ 1919

Foulkes, William ................. 207, 1251

Fowle, Evan J................................ 368

Fox, Sharon ................................. 1584

Foye, Adam .................................... 934

Fraer, Mony ................................... 1645

Fraig, Mostafa M ................ 493, 1393

Frampton, Garrett........ 496, 790, 994, $1005,1015,1138,1297,1331$, $1654,1836,1855,1880,1920$,

1924

Franchi, Alessandro............... 46, 1292

Franco, Catalina ............................... 1475

Frank, Gina Liviana ..................... 1435

Frank, Renee ........... 319, 1200, 2141

Frankel, Arthur............................. 1508

Frankel, Wendy L........ 655, 694, 746,

$796,803,1696$

Franklin, Donald .......................... 1633

Fraser, Richard ................................ 550

Frauenhoffer, Elizabeth E ........... 1093

Frazier, Shellaine R........... 958, 2053

Frederick, Paul D ........................ 2032

Freed, Darren .................................. 1914

Freedman, Orit ............................. 1180

Freitas, Leandro LL....... 862, 863, 864

French, Christopher...................... 1926

French, Samuel W........................ 1482

Fried, Karen .................................... 352

Friedman, Joel............ 908, 909, 1068

Friedman, Kenneth......................... 831

Friedman, Lisa ............................. 1876

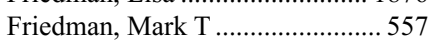

Frierson, Henry F ........................... 889

Frigola, Gerard .............................. 1348

Frishberg, David..................... 504, 505

Fritchie, Karen ........... 31, 39, 76, 500

Frizzell, Kimberly ......................... 1477

Fromm, Jonathan.......................... 1399

Frosina, Denise ………………….... 514

Frost, Marlene …………………........ 264

Frydenlund, Noah ............................. 503

Frye, Joseph S .............................. 504

Fu, Kai........................... 1380, 1527

Fu, Xiujun .......................... 1585, 1992

Fu, Yumei ........................................ 145

Fuchs, Charles.................................. 1803

Fuda, Franklin ................... 1484, 1508

Fuentealba, Patricia...................... 1774

Fuhrman, Kit ................................ 1546

Fujita, Kazutoshi .............. 1018, 1059

Fukatsu, Akitoshi ......................... 1067

Fukayama, Masashi....................... 662

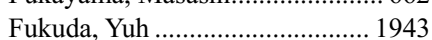

Fukuhara, Noriaki .......................... 617

Fukunaga, Masaharu .........1145, 1146,

1147
Fukuoka, Junya ..... 1909, 1955, 1958,

2001

Fukushima, Noriyoshi...... 1796, 1798

Fulford, Paul .................................... 762

Fullen, Douglas .............................. 497

Fuller, Gregory N ........................ 1721

Fuller, Maren...........................369, 551

Fulmer, Clifton G......................... 1670

Fulton, Regan.......... 966, 1062, 2026

Fung, Po Chu ................................... 334

Furlan, Daniela................................. 819

Furler, Chase V ............................ 2130

Furrer, Daniela .............................. 1962

Furth, Emma E ……………... 709, 808

Furuya, Mitsuko ................................ 58

Fusco, Nicola .............................. 1152

Fuster, Carla ...................................... 359

Fyfe-Kirschner, Billie ...................... 11

Gaafar, Ayman.............................. 1409

Gabbiani, Giulio.............................. 321

Gaber, Germaine ......................... 1329

Gabrielson, Ed................... 1850, 1948

Gadde, Ramya........... 156, 157, 1077

Gagné, Adréanne .......................... 1987

Gagné, Andréanne........................... 1925

Gainor, Justin F ……................... 2012

Gaisa, Michael .............. 406, 407, 485

Gakinya, Samuel ......................... 1547

Galateau, Francoise...................... 1980

Galbo, Filippo D ................................ 629

Galera, Pallavi Kanwar ................ 1400

Gales, Jordan M ........................... 1734

Gallagher, Michael...................... 1860

Gallagher, Torrey L ....................... 2129

Gallan, Alexander ........... 1625, 1980

Gallego, Soledad ........................... 1409

Galli, Susana .................................. 1874

Galliano, Gretchen ............................. 75

Gallinger, Steven.................. 791, 1835

Galuppini, Francesca................... 1486

Galvis Castro, Karen T................. 1401

Gambarotti, Marco............... 46, 47, 83

Gambato, Martina ........................ 1685

Gan, Qiong..............1148, 1149, 1150

Gandara, David R......................... 1907

Gandhi, Manoj ....... 1678, 1679, 1693

Ganesan, Raji ....................1113, 1151

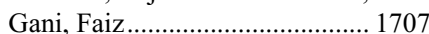

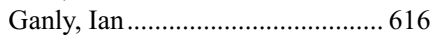

Gao, Chen .................................... 875

Gao, Dana ................................................. 1317

Gao, Dongxia ............................... 1787

Gao, Faye …………........................... 465

Gao, Juehua......................... 711, 1842

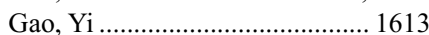

Gao, Yizhuo ………………......... 1984

Garanito, Marlene ............. 1450, 1451

Garber, Judy .................................... 202

Garces, Sofia ..................... 1402, 1403

Garcia, Christopher....................... 1594

Garcia, Ciro....................... 1767, 1768

Garcia, Elizabeth.....1162, 1163, 1212

Garcia, Felip................................... 223

Garcia, Joaquin ................ 1716, 2116

Garcia, Marta ................................ 890

Garcia, Rochelle............... 1226, 1248

Garcia-Bragado, Federico ........... 1409

Garcia-Buitrago, Monica .... 388, 400,

475,1783

García-Domínguez, Daniel J...... 1870

Garcia-Escolano, Marta ....... 158, 159 
Garcia Hernandez, Felip ............... 222 Garcia-Manero, Guillermo.......... 1416 Garcia-Martinez, Araceli...... 158, 159 García-Mejías, Rosa..................... 1870 Garcia-Moliner, Maria L........... 1917, 1968

Garcia-Tsao, Guadulupe.............. 1713 Gardner, Jerad M........................... 551

Gardner, Juli-Anne.............. 666, 1413 Gardner, Paul .............................. 1318

Gardner, Tracie............................. 2114

Garg, Karuna ......................1108, 1188

Garg, Shipra .......................... 35, 1991

Garg, Swati.................................... 1185

Garrett, Erin ............................... 2041

Garrido, Marta................... 1409, 1875

Gars, Eric ..................................... 1404

Garzon, Angie V .......................... 1574

Gascoyne, Randy .............. 1390, 1526

Gasparotto, Daniela.......................... 81

Gast, Katherine ............................... 151

Gaston, Daniel........................ 738, 807

Gaston, Elizabeth .............................. 27

Gatalica, Zoran............................. 1989

Gates, Peggy .................................. 1989

Gattuso, Paolo ....... 1235, 1283, 1308,

$1325,1616,1981$

Gaut, Joseph.......... 1620, 1636, 1650

Gawelek, Kara................................. 160

Gay, Laurie M .................... 196, 238, 496, 790, 994, 1005, 1015, 1138,

1297, 1331, 1654, 1836, 1855, $1880,1920,1924$

Gay, Nick J................................... 1834

Gay-Bellile, Mathilde ................. 1107

Gazzola, Anna ............................... 1486

Ge, Yimin .............................. 370, 380

Gedikoglu, Gokhan M ................... 61

Gehlbach, Daniel.......................... 1508

Geisinger, Kim R ....... 412, 547, 2024

Geisler, Daniel L ........................... 1876

Gejman, Roger .............................. 1533

Geldenhuys, Laurette ................... 2091

Geller, Matthew .............................. 986

Geller, Rachel............... 894, 910, 911

Gellert, Lan L................................. 1083

Gelwan, Elise ............................... 1302

Genco, Iskender Sinan ................... 968

Gener, Melissa AH........................ 1736

Gentry, John D ............................ 1955

Genty, Carlos.................................. 303

Genzen, Jonathan .............. 2021, 2067

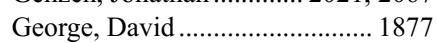

George, Eva..................................... 505

George, Sudeep P.......................... 1863

George, Tracy........ 1365, 1384, 1452,

Geromes, Ariana B....................... 1303

Gersch, Christina............................. 123

Gershenwald, Jeffrey E.................. 533

Gesztes, William R...... 871, 872, 912,

1076

Gettler, Kyle................................. 842

Geyer, Felipe....... 161, 207, 220, 242,

$1152,1312,1854$

Geyer, Felipe C ............................. 243

Geyer, Julia T .................................. 1369, 1405

Ghabril, Marwan .......................... 1694

Ghaffari, Abdi ……..................... 257

Ghaffarieh, Alireza....................... 1735

Ghai, Ritu

1235,1325
Ghai, Sangeet …......................... 1835

Ghanaat, Mazyar ........................... 874

Ghanem, Tamer .......................... 1340

Ghanim, Marcel T....... 113, 139, 175,

1092,1131

Ghiaseddin, Ashley P................. 1742

Ghildyal, Abhilasha..................... 2017

Ghosh, Abheek ............................ 1795

Ghosh, Toshi .......... 1626, 1782, 2094

Ghoshal, Kalpana ........................ 1696

Ghossein, Ronald ........ 447, 581, 582,

$615,616,1343$

Gi, Toshihiro ................................... 838

Giacomelli, Luca........................... 595

Gianduzzo, Troy........... 884, 885, 886

Giannico, Giovanna .......... 913, 1083,

Giannini, Caterina ....................... 1750

Giardiello, Francis............................ 680

Gibb, Ewan A.................... 930, 1056

Gibril, Manal................................. 891

Gibson, Briana ............................. 1406

Gibson, Christopher J....... 1542, 1543

Gibson, Ian W ............................ 1638

Gibson, Joanna ............................... 653

Gibson, Sarah E ..... 1347, 1458, 1459

Gibson, William .................... 686, 687

Gilbert, Natalie............................ 1605

Gilks, Blake...1113, 1151, 1213, 1250

Gilks, C Blake....... 1177, 1201, 1218,

1253

Gill, Anthony J ..................... 831, 848

Gill, Peter G .................................. 153

Gill, Ryan ............................... 37, 1677

Gill, Ryan M .................... 1681, 1692

Gill, Sharlene ................................ 722

Gillespie, Theresa W...................... 927

Gilliland, Mary........................... 8, 18

Gilmore, Hannah................. 160, 1864

Gimenez, Cecilia E .................... 2077

Ginter, Paula........ 136, 137, 225, 226,

$\begin{array}{rr} & 227 \\ \text { Giordano, Carla } & 1723\end{array}$

Giordano, Thomas J ...................... 590

Giorgadze, Tamar...... 329, 958, 1594,

2081

Girgis, George ............................ 1395

Giuste, Felipe O ............................. 988

Glaser, Adam K...... 1014, 2080, 2137

Glass, Carolyn H........ 314, 315, 1926

Glass, LF .................................. 1421

Glasser, Daniel .............................. 219

Gleeson, Noreen............................. 1860

Gleeve, Martin ................................ 934

Glenn, Martha J............................. 1520

Glisson, Bonnie............................ 1290

Glover, Michael ........................... 1016

Gnepp, Douglas............................ 1292

Go, Christopher............................. 1275

Go, Ronald S...................... 1641, 1642

Gobbo, Stefano ....................................... 869

Gocke, Christopher D ......... 43, 1555

Goda, JayantSastri....................... 1762

Godleski, John.............................. 1931

Goebel, Emily ............1153, 1154, 2042

Goecks, Jeremy .............................. 995

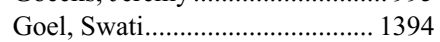

Goerke, Dayna M.......................... 590

Goggins, Michael G ....................... 761

Gogineni, Swarna......................... 1464

Gohlke, Amanda ..................... 16, 914
Gokmen-Polar, Yesim ......... 109, 163,

1978

Goldberg, Michael E........ 1138, 1920

Goldberg, Richard M ................... 722

Goldblum, John R ....... 500, 654, 664, 692, 705, 726, 776, 785, 2133

Goldenberg, David ..... 612, 613, 1342

Goldsmith, Jeffrey D....... 2019, 2068,

2069

Goldstein, Leanne …………….... 1519

Goldstein, Steven ......................... 1121

Golijanin, Dragan.......................... 2108

Golijanin, Jovana .......................... 2108

Gollamudi, Jay ............................. 1603

Gollapalle, Esha ................. 818, 1559

Golombos, David M...................... 978

Golovyuk, Alexander .................. 1845

Gomes, Marcio M ........................ 1904

Gomez, Adam .............................. 676

Gomez, Adam J................... 371, 688

Gomez, Carmen ......... 400, 475, 1292

Gomez, Felicia ............................ 1496

Gomez-Fernandez, Carmen ........ 195, $388,1328,2133$

Gomez-Gelvez, Juan ........ 1426, 1525

Gomolčáková, Barbora ............... 1178

Gondek, Lukasz ............................ 1555

Gondim, Dibson D...................... 1736

Gong, Shunyou ............................ 1453

Gong, Yuna...

.. 1783

Gong, Zimu ............ 1374, 1407, 1408 1423,1556

Gonzales-Ericcson, Paula ............ 164

Gónzalez, Agustin I....................... 1533

Gonzalez, Dianelis ........................ 303

Gonzalez, Jorge L...............1133, 1134

Gonzalez, Juan ..

.. 1833

Gonzalez, Maria ............................ 412

Gonzalez, Raul S........................ 683, 686, 699, $773,786,804,1669$

González-Cantú, Yessica............. 1671

González-Farré, Blanca... 1348, 1409,

Goode, Benjamin ....................... 1753

Goodman, Abigail L.......... 915, 1304

1737,1759

Goodman, Eli ............................... 1471

Goodman, Mark M.......................... 998

Goodman, Michael M......... 417, 432,

Goodwin, Andrew ...................... 2034

Goodwin, Michele T ................... 2059

Goodwin, Pamela J ........................ 275

Gopalan, Anuradha ............. 850, 874, 916, 935, 985, 1037, 1038, 1053,

1055,1058

Gopinath, Arun

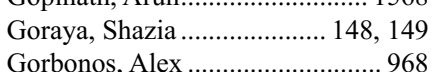

Gordetsky, Jennifer ........ 95, 878, 893, $905,918,1032,1041,1406$

Gordon, Ilyssa ....................... 705, 829

Gordon, Laura .............................. 1599

Gorlick, Richard G..................... 1867

Goswitz, Joseph J............................ 21

Gotlib, Jason ......... 1384, 1437, 1505,

1506

Gotlib, Jason R........................... 1516

Goto, Akiteru.............1156, 1182, 1249

Goto, Takahiro ................................. 71

Gotoh, Momokazu .

... 1067
Gottlieb, Chelsea................. 146, 1155

Gottlieb, Jens............................... 1945

Goudie, Marissa .............................. 807

Gougeon, Francois ....................... 1627

Gown, Allen M......... 913, 966, 1062,

2026

Goyal, Bella ................................. 1557

Goyal, Kashika............................ 1007

Goyal, Rajen ………………....... 2123

Goyal, Tanu ....................... 1410, 1411

Goytain, Angela ........ 40, 1213, 1295 ,

1869

Grabenstetter, Anne ........................ 165

Grada, Zakaria....................917, 1412

Gradecki, Sarah E ...................... 2138

Graham, Mindy K ....................... 1772

Graham, Rondell ....... 382, 689, 1671, $1680,1782,1786,1808,1986$,

2084

Graham, Tiffany ..................... 95, 918

Grandori, Carla ........................... 1853

Grange, Jacob............................... 1927

Grant, Michelle L.......................... 1413

Granter, Scott R............................ 1576

Gratzinger, Dita................................... 396

Grau, Ana ......................................... 164

Grauslund, Morten ........................ 1284

Gravet, Claire .............................. 1973

Gray, Alice L ................................. 1902

Green, Andrew …................. 252, 260

Green, Peter HR .............................. 723

Green, Ralph ............................... 1383

Greenland, Nancy …...................... 919

Greenwood, Michelle................... 1042

Greer, Adam H ........................... 2154

Gregory, Kelly J ............................ 1841

Greiner, Timothy ........................... 1527

Greipp, Patricia T ............ 1396, 1494,

1671,1782

Grenert, James....................... 199, 826

Grewal, Mandeep ........................... 1633

Griff, Sergej ................................ 1955

Griffin, Brannan ................. 166, 1877

Griffin, Laura ................... 2119, 2121

Griffith, Brent.............................. 1760

Griffith, Christopher........................... 1269

Griffith, Christopher C........ 372, 479, $599,1170,1304,1336$

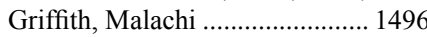

Griffith, Obi................................. 1496

Grignon, David ....... 561, 1003, 1004, $1021,1031,1033$

Grillo, Federica ............................... 819

Grobmyer, Stephen R........... 148, 149

Groen, Ruben ……….................... 1958

Grönberg, Henrik ........................... 895

Gronchi, Alessandro......................... 81

Grondin, Katherine ....................... 1218

Groover, Surbhi.............................. 235

Grosjean, Fabrizio........................... 1628

Gross, Sara ........................................55

Grosset, Andrée-Anne..... 2106, 2125 ,

2152

Grossmann, Petr................... 70, 1054

Groth, John................................... 1179

Grotto, Paolo ................................. 1750

Gru, Alejandro............................... 539, 889

Grunes, Dianne E............... 412, 2024

Grzybicki, Dana M....................... 2024

Gu, Dongqing........................................ 492

$\mathrm{Gu}$, Xiaoping.................................. 163 


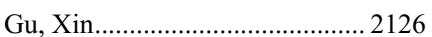
Guajardo, Andrew .......................... 325 Gucer, Hasan .................................. 579 Gudi, Mihir ............................. 129, 167 Gudino, Cynthia ............................. 611 Guerra, Laura ................................. 1875 Guerra, Marjorie-Anne R............. 690 Guetter, Christoph ........................ 2114 Guha, Sushovan .................. 630, 1819 Guidry, Joseph T ……................. 1859 Guillen, Francisco ........................ 1739 Guillory, Bryan ............................. 188 Guimaraes, Gustavo C .... 1027, 1028, 1029

Guindi, Maha .............................. 1658 Gularte-Merida, Rodrigo............ 1312 Gulati, Rohit................................ 2043

Gulati, Roman ............................... 1066

Gulavita, Previn ...........................1117

Güler Tezel, Gaye Y........................ 61

Guma, Sergei R..................... 48, 920

Guner, Gunes ................................. 993

Guo, Ailin.................................... 1862

Guo, Chao …………………….... 1081

Guo, Charles .............. 883, 933, 1071

Guo, Hua ........................................... 168

Guo, Ling ......................... 1414, 1415

Guo, Ruifeng ..................................506

Guo, Shunhua.............................. 1629

Guo, Tao............................................. 169

Guo, Tianhua.................................... 145

Gupta, Anika ………….................. 1924

Gupta, Arjun ................................ 1378

Gupta, Chhavi ...................................... 80

Gupta, Divya ................................ 1204

Gupta, Ekta .................................. 293

Gupta, Gopal ......................... 936, 937

Gupta, Nilesh S ......... 221, 943, 1044,

1065,1077

Gupta, Raavi ........... 707, 1436, 1609

Gupta, Rajib ................................ 1672

Gupta, Ruta ........... 1305, 1311, 1330,

1333

Gupta, Sameer........................... 1802

Gupta, Sanjay .................... 1016, 1091

Gupta, Sounak..... 900, 921, 922, 923, $931,967,1662$

Gupta, Tejpal ............................... 1762

Gur, Deniz .............. 1353, 1416, 1445

Guragain, Deepti Adhikari ............ 490

Gurav, Mamta .............................. 1762

Guseva, Natalya ..................... 54, 384

Guy, Cynthia ............ 693, 1043, 1701

Guzman, Grace ...... 1838, 2148, 2150

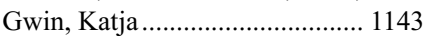

Haas, Mark ……………………..... 1623

Haba, Reiji .................................. 1936

Habeebu, Sultan ................................ 85

Habermann, Thomas M............... 1652

Hacihasanoglu, Ezgi ............ 417, 432,

1784

Hackeng, Wenzel.............................. 761

Haddad, Zaid............................... 1085

Hadi, Rouba ................................... 1673

Hadravska, Sarka ................................ 7

Hadravsky, Ladislav..... 70, 984, 1207

Haeri, Mohammad ............ 1306, 1524

Hafez, Khaled S ................ 1049, 1069

Hafez, Omeed ................................. 299

Hafezi-Bakthiari, Sara.................... 712

Haffner, Michael C......................... 860
Haga, Hironori ............................... 1957

Hagemann, Andrea R ................... 2044

Hagemann, Ian .......1175, 1190, 2044,

2093

Hagen, Catherine......... 621, 651, 717,

752,753

Haghighi, Mehrvash................... 1928

Hagiya, Ashley S.......................... 1461

Hahn, Elan....................................... 867

Hahn, Jamie....................... 1530, 1536

Haimes, Josh ...........1189, 1295, 2119

Haines, G Kenneth ......................... 854

Hajdu, Cristina .................................... 695

Hajifathalian, Kaveh ..................... 664

Hajiyeva, Sabina ................ 924, 1565

Hakim, Shaheed W ........................ 691

Hakima, Laleh.................................. 335

Hakimi, Ari ..................................... 874

Halani, Sameer H......................... 1760

Haley, Lisa ................................... 1555

Halimi, Sultan A................ 1156, 1249

Hall, Curtis R .................................. 778

Hall, Sean R ................................. 1959

Haller, Florian .............................. 2149

Halling, Kevin.................................. 535

Halloran, Philip .......................... 1614

Halushka, Marc ............................... 325

Hamad, Eman.................................. 312

Hamadain, Elgenaid...................... 1412

Hamadeh, Fatima T........................ 373

Hamasaki, Makoto ............ 1942, 2011

Hameed, Meera ......................... 65, 79

Hameed, Omar ......... 169, 1083, 1132

Hamele-Bena, Diane ....................... 571

Hamilton, Ronald L..................... 1730

Hammad, Abdulrahman .............. 1655

Hammad, Nazik .............................. 643

Hammer, Richard D ......... 1371, 2053

Hammer, Suntrea TG .......... 670, 726,

1818

Hammond, Scott ............................ 1611

Hamoudi, Rifat.............................. 1805

Hampel, Heather ..................... 694, 746

Han, Ho-Seong............................. 1792

Han, Kathy …………………….... 1180

Han, Lisa ................... 170, 925, 1157

Han, Liying ........................ 1025, 1274

Han, Lucy........................................ 171

Han, Min ......................................... 2044

Han, Xiao ...................................... 170

Han, Xin ........................................ 1556

Hang, Jen-Fan ............. 374, 375, 402

Haninger, Diana ............................ 1523

Haninger, Diana M........................ 1395

Hankinson, Sue ............................... 117

Hanley, Krisztina Z ..... 362, 417, 432, $558,1228,1252$

Hanlon-Newell, Amy .................... 122

Hanna, Matthew G.......... 1586, 1587,

1588,1590

Hanna, Mereet.................................. 891

Hanna, Wedad .................................. 231, 298

Hannibal, Charlotte G ................ 1271

Hansel, Donna..... 921, 923, 960, 969, $991,1051,1080,2140$

Hansen, Katrine.......... 247, 360, 1273

Hanson, Curtis A .......................... 1554

Hanson, Joshua A....................... 25, 49

Hanson, Paul J................................. 316

Hansson, Magnus ................................ 34

Hao, Xueli ........................... 985, 1055
Haque, Monira .............................. 2127

Hara, Shigeo................................. 1632

Haraldsdottir, Sigurdis .................. 694

Hardin, Heather... 573, 574, 575, 591,

592,618

Harigopal, Malini........ 133, 294, 299 ,

308
Harinath Lakshmi $\quad 376,926$

Hariri, Lida......................... 1992, 1993

Hariri, Nosaibah ........... 172, 173, 174

Harmon, Maureen ..............1115, 2034

Harpaz, Noam ..... 692, 729, 730, 731, $736,737,742,843,844,1801$,

Harper, Aaron E

Harper, Holly L ............ 979, 980, 981

Harrell, Danielle........................... 2045

Harris, Nancy ..................... 1363, 1513

Harris, Wayne B ............................. 927

Harrison, Beth T...........................1113

Harrison, Grant ............ 389, 580, 693

Harrison, Thomas......................... 2121

Harshyne, Larry A.......................... 1733

Hart, Eric...................................... 1906

Hart, John.... 415, 628, 629, 718, 719,

1656,1673

Hartley, Christopher.... 346, 377, 605 , $621,651,717,752,753,1020$

Hartman, Anne-Renee................... 518

Hartman, Douglas ............... 1588, 1589

Hartmann, Arndt ......... 848, 881, 984 $1065,1282,2149$

Hartz, Brian.................................... 21

Harzman, Alan ………..................... 803

Hasebe, Osamu ............................ 1794

Haseeb, MA........................ 1436, 1609

Hashisako, Mikiko ............. 1909, 2001

Hassan, Mohamed............... 527, 1393

Hassan, Muhammad...................... 1648

Hassan, Oudai ............................... 928

Hassanpour, Saeed ............ 1599, 1599

Hasserjian, Robert P........ 1381, 1452, $1542,1543,1546,1547$

Hasteh, Farnaz.... 172, 174, 181, 1865

Hata, Shuko................................... 1982

Hatakeyama, Kinta............................ 1632

Hatanaka, Yutaka .......................... 1817

Hatanpaa, Kimmo …………....... 1725

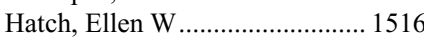

Hatem, Joseph ................................. 490

Hathuc, Vivian .................................... 1878

Hathuc, Vivian M........................ 2046

Hattab, Eyas M.................................. 1736

Hattori, Ryohei..................................... 1067

Haugg, Anke M................................. 509

Haun, Paul...................................... 1493

Have, Cherry ............................... 1126

Hawkins, Jaleah ............................... 2047

Hawley, Dean A ................................. 24

Hayajneh, Rami...................................... 1838

Hayashi, Hiroyuki ........................ 1033

Hayashi, Kentaro........................... 1958

Hayashi, Takuo............................... 317

Hayek, Kinda ............ 175, 1092, 1094

Hayes, Daniel F............................. 123

Hayes, Malcolm ............................. 1295

Hazari, Rakesh S............................... 516

He, Hong-Lin ................................. 929

He, Huiying ................................. 1054

He, Jian ............................. 188, 2128

He, Jing ..................................... 1345
He, John ........................................... 769

He, Rong ...... 1396, 1397, 1512, 1554

He, Xin............................... 176, 542

Head, David R.................. 1504, 1510

Healy, Marie Louise.................... 1326

Heaphy, Christopher M...... 853, 1772

Heath, Jonathon E ........................ 1995

Heather Eliassen, A .......................... 117

Hebisawa, Akira ............................ 1943

Hecht, Jonathan ................................ 9

Hechtman, Jaclyn......... 668, 732, 733

Hechtman, Jaclyn F..................... 1837

Hedayat, Amin A............................ 2129

Hedley, David .......... 638, 1789, 1835

Hedley-Whyte, E Tessa................... 22

Hegazy, Samar ............................... 930

Heher, Yael K ................................ 2019

Hein, Ashley H............................... 1527

Hekman, James ............................... 392

Helderman, Harold.......................... 1633

Heller, Theo....................................... 626

Hellmann, Matthew....................... 2003

Hemmerich, Amanda ........................... 693

Hemminger, Jessica ......................... 694

Henderson, Les J........... 979, 980, 981

Henderson, Rosemary ................. 2091

Henderson-Jackson, Evita................. 50

Hendrickson, Heather ..................... 369

Heng, Yujing Jan ............................. 117

Hennessy, Max ....................... 613, 1342

Henricks, Walter............................. 705

Henriet, Julien ....................... 507, 508

Henriksen, Jonathan ........................ 219

Henriksen, Kammi J......... 1618, 1621 ,

Henry, Elizabeth $\quad 1625$

Henry, Michael....................................... 1905

Hensley, Marie ................................ 119

Hensley, Martee ……………….... 1125

Heo, Su Jin ................................... 1296

Heras, Alfonso............................... 2130

Herculiani, Amanda P ........ 862, 863,

Herfs, Michael............................... 1243

Herman, Carolyn R ....................... 224

Herna, Stuart J................................. 1328

Hernandez, Andrea........................... 307

Hernandez, Francisco.................... 1875

Hernandez, Luz Andrea .............. 1574

Hernández-Iglesias, Teresa ........... 183

Hernandez-Losa, Javier................. 1983

Hernandez-Perez, Marier .............. 503

Hernandez-Prera, Juan ..................... 582

Herrera, Alex................................... 1519

Herrera, Guillermo A ......... 487, 1630 ,

2118,2126

Herrera, Mileyka ........................... 1534

Herrera Hernandez, Loren P ...... 923, $931,967,1619,1626$

Herring, Nicole ................................ 14

Hes, Ondrej ......... 849, 881, 983, 984,

1054,1066

Hes, Ondřej ........................ 848, 1065

Hess, Paul...................................... 1356

Heusel, Jonathan W........................ 1190

Hexner, Elizabeth......................... 1356

Heymann, Jonas ……………................ 1965

Heymann, Jonas J.......................... 1928

Hezel, Aram ……………………... 1803

Hickey, Tyler BM............................. 10

Hickman, Richard A.............. 647, 695 
Hicks, David .......................... 191, 287 Hicks, Jessica ..... 124, 859, 993, 2113 Hicks, John....................... 1306, 1879 Hickstein, Dennis D .................... 1358

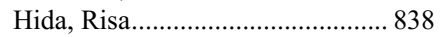
Hida, Tomoyuki ................ 1942, 2011 Hidalgo, Juliana ........................... 1417 Hidalgo Lopez, Juliana E........... 1418 Higgins, Brandi ................. 1895, 2099 Higgins, John P ........ 589, 1698, 1699 Hijazi, Nouf.................................. 956 Hill, Charles E......... 372, 1550, 1759, 2146

Hillen, Lisa....................................... 509 Hiltzik, David.................................. 571

Himmat, Sayed.............................. 1914

Hing, Sandra ................................. 1320

Hinson, Stacy ................................. 2049

Hirahashi, Minako......................... 838

Hirakawa, Akihiro........................ 1067

Hirano, Satoshi............................ 1817

Hiraoka, Nobuyoshi .............. 98, 1954

Hirokawa, Mitsuyoshi................... 610

Hiroshige, Karina ........................... 682

Hiroshima, Kenzo ............. 1942, 1980

Hirsch, Michelle S .... 949, 950, 1162, 1163, 1211, 1242, 1809

Hisa, Takeshi ................................ 1794

Hiser, Wesley ................................. 1738

Hishima, Tsunekazu .......................... 71

Hiskey, Matthew ............................ 177

Hissong, Erika ............................... 669, 696, 697

Ho, Bridget.................................... 593

Ho, Donald M-T........................... 1724

Ho, Ken ........................................... 232

Ho, Khek Yu................................... 813

Ho, Wanting ...................................... 1448

Hoang, Lien....................... 1244, 1245

Hoang, Lien N.............................. 1126

Hoang, Mai ..................................... 503

Hobenu, Frederick......................... 1097

Hoch, Benjamin .............................. 513

Hoda, Raza S........................ 378, 438

Hoda, Syed A ....................... 126, 246

Hodges, Kurt ....................... 150, 1488

Hodgson, Anjelica.............1158, 1159,

1160,1161

Hodjat, Parsa ............ 510, 1168, 1423

Hodkoff, Alexey A .......................... 818

Hodos, Jaccob .............................. 1342

Hoffman, Robert ................................ 28

Hoffmann, Jenny C ......... 1419, 1420,

Hogg, Melissa .................... 467, 1813

Hohenwalter, Mark ...................... 2131

Holcomb, Kevin ............................ 1204

Holder, Chad A............................. 1760

Holland, Steven M ....................... 1358

Hollmann, Travis J ......................... 514

Hollyfield, Johnathan ........................ 379

Holman, Carol............................... 1503

Holmes, Chris ................................ 260

Holmes, Margaret............................. 178

Holtorf, Heidi ................................... 380

Homan, Suzanne ............... 1881, 2162

Homer, Robert J ........................... 1900

Hon, Jane Date ...................................... 11

Honap, Sayali N ............................ 794

Honda, Takayuki ........................... 758

Hong, Aram .................................. 1565

Hong, David ..................... 1922, 2040
Hong, Jie ......................................... 1825

Hong, Kuick Chik .......................... 167

Hong, Seung-Mo............... 1778, 1790

Hong, Sung-Hyeok ...................... 1874

Hong, Yin .................................... 2154

Hontecillas, Lourdes .................... 1870

Hoogestraat, Daniel....................... 1558

HooKim, Kim ...................... 381, 382

Hooper, D Craig ........................... 1733

Hooper, Jody E.................................. 12

Hopkins, Benjamin D................. 1853

Hopkins, Mark ………………........ 426

Hopper, John ................................... 792

Hora, Milan........................ 983, 1054

Horbinski, Craig M..................... 1730

Horigome, Naoto.......................... 1794

Horiguchi, Shinichiro....................... 71

Horna, Pedro ................................. 1375, 1421

Hornick, Jason L ....... 41, 52, 84, 588, 1211, 1233, 1310, 1509, 1577,

1803,1884

Horowitz, Neil S ......................... 1212

Horton, Bethany J ......................... 2138

Horton, Janet K ............................... 244

Horton, Meredith........................... 1874

Horvai, Andrew E ....... 31, 37, 73, 74,

Horvath, Bela ............................. 698

Hosameddin, Mobashir ............... 1838

Hosfield, Elizabeth................ 199, 261

Hoskin, Tanya ................................. 264

Hoskin, Victoria …………................... 257

Hosny Mohammed, Kareem ........ 932

Hosseini, Mojgan ........ 650, 727, 826,

1656,1686

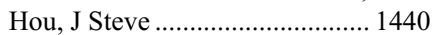

Hou, Le .......................................... 1613

Hou, Shuang ................................... 2161

Hou, Yanjun ........... 179, 180, 212, 213

Hovelson, Daniel............................. 882

Hovelson, Daniel H............. 123, 1069

Howard, Matthew T .......... 1396, 1397

Howarth, David J ............................ 538

Howell, David N ........................... 1902

Howitt, Brooke E ...1162, 1163, 1210,

$1212,1222,1242$

Hrada, Kenichi ............................ 1684

Hruban, Ralph H ................. 761, 1772

Hruska, Chad J ............................... 933

Hruszkewycz, Andrew .................. 853

Hsi, Eric D ... 1360, 1410, 1411, 1414

Hsiao, Susan J .................... 783, 1489

Hsieh, James J................................... 874

Hsu, Amy .................................... 1358

Hsu, Chih-Yi ................................. 1724

Hsu, Ying-Han R .......................... 1929

Hu, Buqu ..................................... 772

Hu, Jianhua ................................... 1846

$\mathrm{Hu}$, Jingjing ...............181, 1164, 1165

$\mathrm{Hu}$, Shaomin .............................. 1839

Hu, Shimin ............ 1357, 1359, 1373,

1374, 1407, 1408, 1423, 1501,

$1556,1922,2040$

$\mathrm{Hu}$, Zhihong .......... 1353, 1357, 1422,

1423,1521

Huang, Anne .................................. 826

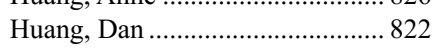

Huang, Danning ................... 661, 817

Huang, Eric C................................ 387

Huang, Fangjin.............................. 1856
Huang, Hongying .... 856, 1000, 1072, $1073,1089,1090$

Huang, James ................................. 1497

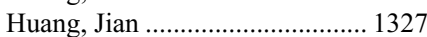

Huang, Jiaoti ................................... 934

Huang, Kuo-Cheng ............... 935, 985

Huang, Marilyn .................. 1217, 1221

Huang, Qin ....................... 1387, 1623

Huang, Richard SP..................... 1488

Huang, Shih-Chiang......................... 56

Huang, Weei-Yuan ........................... 807

Huang, Wei........... 618, 979, 980, 981

Huang, Weihua ........ 924, 1023, 1025 ,

1563,1565

Huang, Wei-Yuarn............................ 738

Huang, Xiao …………………….... 383

Huang, Xiao-Jun ........................... 1538

Huang, Yajue...

... 620

Huang, Yanfei............ 654, 1674, 1675

Hubbard, Elizabeth W................. 2055

Hubbard, Gretchen K .................... 853

Huber, Aaron R ...................... 699, 804

Hudis, Clifford .......................... 285, 286

Hudnall, Stanley D....................... 1481

Hudson, Chad.................................. 1424

Hue, Susan Swee Shan................. 184

Huerta-Yepez, Sara ....................... 1868

Hugar, Sarah B ............................ 182

Hughes, Ian .................. 936, 937, 999

Hughes, Kenneth T ........... 1631, 1653

Huh, Gi Young ...................... 724, 771

Huho, Albert......................... 1919, 1930

Hui, Ling ......................... 938, 1785

Hui, Pei ............ 331, 652, 1104, 1281 ,

Hui, Yiang ...295, 540, 700, 917, 939

1359
939

Hujber, Zoltan ............................... 1944

Hulick, Peter J .................................... 101

Hull, April M................................... 369

Humayun, Islam .............................. 904

Humphrey, Peter ................. 940, 1066

Hung, Yin (Rex) ... 51, 52, 1268, 1931

Hung, Yin P............. 588, 1884, 1926

Hunt, Bryan.............. 329, 1594, 2081

Hunter, Mary .................................. 550

Hunter, Stephen................ 1737, 1759

Huntsman, David G ..................... 1201

Huo, Lei ................................... 92, 208

Hurlbut, David ............................... 643

Hurrell, Sarah L............................... 2131

Husain, Aliya N...... 1123, 1980, 1981

Husain, Nuzhat....... 1166, 1339, 1802

Hussaini, Mohammad ...... 1517, 1551

Hussein, Deema .............................. 1717

Hussenet, Claire .............................. 1950

Hutchings, Danielle............... 701, 781

Hutchinson, Lloyd............... 461, 1811

Huynh, Tiffany ........ 789, 1963, 1992,

2012

Hwa Kim, Chae............................ 1532

Hwang, Chung Su ................... 724, 771

Hwang, David .............................. 1984

Hwang, David H ........................... 1932

Hwang, E Shelley............................. 116

Hwang, Helena.............................. 2030

Hwang, Jin-Hyeok .......................... 1792

Hwang, Michael J .......................... 940

Hyman, David M ............. 1831, 1837

Hyun, Michael D.......................... 1753

Iacobuzio-Donahue, Christine ... 1861
Iafrate, Anthony ….............. 438, 1585 Iafrate, John............ 1763, 1963, 1977 Ibrahim, Hiba ............................ 1167 Ibrahim, Mohammad.......................526 Ibrar, Warda................................... 1634

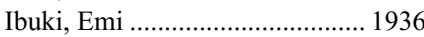

Ichikawa, Hiromi ......................... 1909

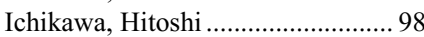

Iczkowski, Ken A............... 845, 2131

Iczkowski, Kenneth........... 955, 1020,

2081

Ide, Hiroki ............................... 941, 951

Idel, Christian................................... 1314

Idoate, Miguel A........................... 1739

Idowu, Michael O ............... 433, 1976

Idrees, Afshan .............................. 1553

Idrees, Mohammad ........... 1003, 1004

Idrees, Muhammad .............. 480, 561, $852,902,974,1010,1031,1032$, $1033,1063,1075,1898$

Ieremia, Eleni............................... 511

Igarashi, Ai ....................................... 764

Iglesias, Mar...................................... 183

Iglesias Coma, Mar ........................ 355

Igor, Frank ....................................... 888

Ikegami, Masahiro ...................... 1040

Ikeri, Nzechukwu Z ......................... 13

Ikpatt, Offiong F........................... 1369

Illan, Francisco J ............................ 158

Illei, Peter............... 1272, 1948, 1979

Imaoka, Yuki .................................. 1958

Imperatore, Kailee............................ 14

Inacio, Joao R............................. 1904

Inaguma, Shingo .......................... 501, 702

Inamdar, Kedar V ............. 1426, 1525

Inghirami, Giorgio G................... 1405

Ingoldsby, Helen ............................. 120

Ingram, Davis R..... 32, 45, 72, 93, 94

Inoshita, Naoko ............................. 617

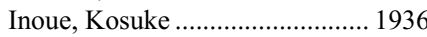

Inoue, Satoshi........................ 941, 951

International Fibroepithelial

Consortium 278

Inwards, Carrie..................................... 39

Inzani, Frediano ........................... 1277

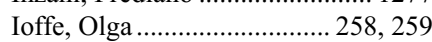

Ip, Philip PC ................................ 1262

Iqbal, Jabed ......... 167, 184, 185, 304,

Iqbal, Javeed $\quad 1840$

Irmak, Yasin ....................................... 1959

Irshaid, Lina ......... 136, 137, 225, 227

Isaacs, William .............................. 860

Isaacson, Alexandra ....................... 552

Isaila, Bogdan ……........................ 2048

Isaji, Shuji ................................... 1816

Isbell, Lauren ............................... 1168

Ishak, Elia A..................................... 945

Ishida, Kazuyuki ............................. 764

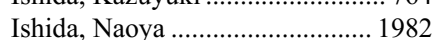

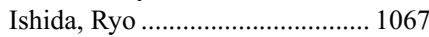

Ishikawa, Ryou........................................... 1936

Islam, Shahid......................453, 1117

Isler, Christy ........................................... 6

Ismail, Mamudo R ....................... 1573

Ismiil, Nadia........................1160, 1161

Isrow, Derek ………........................... 1340

Itami, Hiroe .................................... 1632

Ito, Masaaki..................................... 797

Ito, Tetsuya.................................... 1794

Iuga, Alina ............................. 723, 810 
Ivan, Doina......... 525, 533, 537, 2018 Ivanovic, Marina ............................. 585 Ivins, Stephanie............................... 89 Iwenofu, O Hans .............................. 457 Iyer, Gopa................ 850, 1055, 1058 Iyer, Gopakumar V........................ 985 Izevbaye, Iyare ........ 1261, 1287, 2112 Izimukwiye, Isabelle ..................... 232 Jabbar, Seema B ........................... 2049 Jabbour, Hossam .................... 210, 211 Jaber, Omar ...................... 53, 54, 384 Jackson, Sara...339, 1285, 1822, 2111 Jacobs, Timothy .............................. 261

Jacobsen, Gordon......................... 1340

Jacobsohn, Kenneth ..................... 2131

Jaffe, Elaine S ............................ 1390

Jaffee, Elizabeth ............................. 281

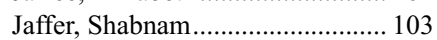

Jagannathan, Geetha ...................... 186

Jaggi, Meena ................................ 1775

Jain, Deepali........... 1566, 1933, 1955

Jain, Dhanpat... 772, 834, 1661, 1705,

1776

Jain, Sarika .................................. 1425

Jain, Shilpa ............................................. 462

Jain, Sudhanshi S .......................... 167

Jakate, Kiran .................................. 691

Jakate, Shriram........... 703, 774, 1604

Jakhi, Tanvi .................................. 2035

Jalali, Rakesh ............................. 1762

Jalaly, Jalal B ............................... 1307

Jalikis, Florencia ........................... 1659

Jamal, Mohsin .............................. 1426

Jamaspishvili, Tamara................... 867

Jameel, Zena …………………..... 1427

Jamshed, Sarah.................... 704, 2087

Janaki, Nafiseh .......... 187, 942, 1740,

1956

Janatova, Marketa .......................... 207

Jang, Erika L .................................. 316

Jang, GunHo ................................ 1835

Jang, Jinyoung................................ 1778

Jang, Kee-Taek................. 1778, 1800

Jang, Kiseok ......................... 254, 255

Janostiak, Radoslav....................... 294

Janovitz, Tyler .................................... 1934

Janowczyk, Andrew .......... 187, 1607,

1864,2141

Jaramillo, Lina E........................... 1401

Jaratli, Hayan ................................. 741

Jardin, Fabrice.............................. 1973

Jaros, Mark................................... 1604

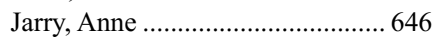

Jattani, Rakhi................................ 1477

Javidian, Parisa .............................. 1605

Javidiparsijani, Sara .................... 1308

Jawad, Hadeel ................................ 1905

Jawale, Rahul ...................... 705, 706

Jawale, Rahul M........................... 1841

Jayakumar, Rajeswari ........... 583, 707

Jayakumaran, Gowtham................ 874

Jazaeri, Amir A...................1193, 1194

Jebakumar, Deborah.............. 102, 188

Jebastin, Judith AS ......................... 943

Jeck, William Richard.................... 708

Jeffreys, Matthew.......................... 1169

Jeffus, Susanne ................................. 1118,1169

Jen, Jin.................................... 74, 689

Jen, Kuang-Yu................................. 200

Jeney, Andras ................................. 1944

Jenkins, Mark ........................ 791, 792
Jenkins, Sarah .... 39, 620, 1929, 2084

Jenkins, Taylor M........................... 709

Jennette, Charles .......................... 1651

Jennette, J Charles.......................... 1627

Jennings, Lawrence.......... 1453, 1877

Jennings, Timothy .......................... 1919

Jensen, Chris ........................ 384, 553

Jensen, Kristin........................111, 112

Jenson, Erik G.............................. 1127

Jentoft, Mark E................................ 38

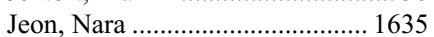

Jeon, Sora .......................................... 584

Jeong, Hyeon Joo ............................ 1635

Jermyn, Michael........................... 2125

Jermyn, Mike .............................. 2152

Jesinghaus, Moritz ...................... 1791

Jessurun, Jose...... 669, 696, 697, 782,

836

Jeung, Jennifer ................................. 705

Jevremovic, Dragan ..................... 1467

Jeyachandran, Devi.......... 1590, 1801

Jeyarajah, Arjun J........................ 1151

Jhala, Nirag ........ 312, 336, 411, 970,

$971,972,1327$

Jhun, Iny ........................................... 976

Jia, Liwei......................................... 385

Jiang, Chunjie ............................... 1389

Jiang, Haiyan................................. 1070

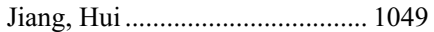

Jiang, Kun .................. 815, 816, 1560

Jiang, Manli................................... 900

Jiang, Wanhong...... 1964, 2143, 2146

Jiang, Wei..................................... 1170

Jiang, Xiaoyin "Sara"......... 389, 1701

Jiang, Xiaoyin S .................. 551, 580

Jiang, Yuying................................. 385

Jibrin, Paul G ................................. 767

Jimenez, Miguel Angel .... 1002, 1047

Jimenez, Rafael E........ 923, 931, 967,

990,1086

Jimenez, Yuis ................................ 1967

Jin, Chunyuan ................................. 725

Jin, Jang............................................ 74

Jin, Jennifer .................................. 1081

Jin, Long ................................ 39, 689

Jin, Ming ..................................... 386,459

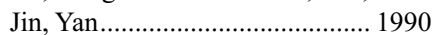

Jin, Yasuto ..................................... 1935

Jing, Xin ............................................ 401

Jiron, Kassandra ........................... 1930

Jo, Vickie Y..... 68, 1310, 1321, 1338

Job, Bastien................................... 473

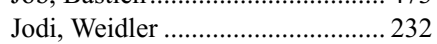

Joehlin-Price, Amy S ....... 270, 1171,

1192

Joël Tremblay Marchand, Joël

Tremblay ................................. 1987

Johannesen, Eric ........................... 2053

John, George …………………..... 1378

John, Veena S ................................ 1195

Johncilla, Melanie ............................ 662

Johncilla, Melanie E............. 710, 1577

Johnson, Adrienne........................ 1880

Johnson, Bruce E ......................... 1932

Johnson, Joseph............................... 603

Johnson, Joshua............................ 2026

Johnson, Laura A................ 2119, 2121

Johnson, Nathalie .......................... 1526

Johnson, Sarah H ......... 922, 923, 931

Johnson, Sheryl L........................ 1172

Johnstone, Sarah E .......................... 42
Jolly, Grant A ................................. 1273

Joneja, Upasana............................. 1741

Jones, Andrea ......................620, 1786

Jones, Andrew D ............................. 387

Jones, Carol.................................. 2114

Jones, David M ..................... 496, 763

Jones, Derek ................................... 1173

Jones, Heather H ................. 372, 2146

Jones, Kirk .................................... 1921

Jones, Martin ................................ 1213

Jones, Mirka W ............................. 1174

Jones, Raymond .............................. 122

Jones, Ryan D ............... 15, 711, 1842

Jones, Terrell ................................. 1591

Jonigk, Danny ............................... 1945

Jorda, Merce........ 195, 388, 400, 475, $956,973,1030,1077,2133$

Jordan, Elaine K............................ 1389

Jordan, Emmet J............................ 1058

Jordan, Richard ............................... 199

Jordao, Dercio ................................ 1573

Jorgensen, Jeffrey L .......... 1422, 1434

Jorissen, Robert................... 827, 828

Jorns, Julie M..... 151, 233, 234, 2046

Jose, Bincy .................................. 2132

Joseph, Nancy .............................. 826

Joseph, Nancy M... 37, 199, 261, 748, $1676,1677,1753$

Jothishankar, Balaji ....................... 512

Joubert, Philippe ... 1910, 1925, 1940 , $1962,1975,1987$

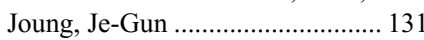

Jour, George ......................... 513, 2018

Jouret-Mourin, Anne ....................... 478

Jovanovic, Bojana .......................... 164

Joyal, Thomas ....................... 388, 400

Juarez-Villegas, Luis E ................. 1868

Judson, Marc ................................. 1919

Jug, Rachel.................................. $1889,1447,1886$

Jung, Chan Kwon.......................... 584

Jungbluth, Achim ...... 242, 514, 1368 ,

Juraszek, Amy

Juskevicius, Ridas ............................ 1510

Kaag, Matthew ............................. 1074

Kabira, Ana-Tereza ...................... 1623

Kacerovska, Denisa.... 520, 521, 1207

Kaczmarowski, Amy.................... 2131

Kadin, Marshall............................. 1428

Kadjacsy-Balla, Andre .................. 230

Kadota, Kyuichi ............................ 1936

Kadri, Sabah.................................. 629

Kagen, Alexander............................ 1801

Kahl, Brad S................................. 1479

Kahlenberg, Morton ......................... 722

Kahn, Ryan.................................. 1221

Kajdacsy-Balla, Andre ................ 1838

Kakar, Sanjay .... 661, 817, 826, 1661, $1663,1676,1692,1705,1708$

Kakizaki, Fumie.

Kakshapati, Trishna.

Kalhor, Neda ..................... 1237

Kalife, Elizabeth T ...... 176, 206, 272

Kalimuthu, Sangeetha N ...... 712, 802

Kalinin, Dmitry ................. 1844, 1845

Kalir, Tamara...485, 1112, 1198, 1590

Kallakury, Bhaskar VS....... 944, 992, 994, 1005, 1895, 2099

Kallen, Michael E

1390

Kalloger, Steve E ......................... 1787

Kalof, Alexandra

. .1115
Kamal, Mohammed.......... 1098, 1099

Kamath, Anitha ............................ 1481

Kambham, Neeraja .......... 1698, 1699

Kambouchner, Marianne............. 1950

Kamdar, Kala ................................ 1379

Kamel, Dhay ……………………........ 395

Kamel, Mohamed K......... 1937, 1938

Kamel, Nora N ............................. 945

Kamel-Reid, Suzanne ......... 466, 802,

1185

Kamen, Diane L ............................ 1526

Kammerer-Jacquet,

Solene-Florence ..... 946, 947, 948

Kamoun, Malek.............................. 319

Kamran, Waseem ......................... 1860

Kanaan, Hassan D ........................ 2027

Kanaan, Yasmine.................... 192, 193

Kanagal Shamanna, Rashmi ...... 1417

Kanagal-Shamanna, Rashmi..... 1429,

1434,1488

Kanagasabapathy, D Anand

Rajan .....

Kanaji, Nobuhiro....

Kandel, Rita A........ 86, 88, 538, 2151

Kandil, Emad ………...................... 527

Kandukuri, Shivani ............... 949, 950

Kane, Christopher ......................... 991

Kane, Shubhada ...... 634, 1289, 1311, 1324

Kane, Yehonatan .......................... 1592

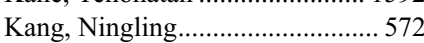

Kang, Yuna.................................... 1176

Kanthan, Rani ............................. 1105

Kao, Chia-Sui.......... 589, 1003, 1032,

1124,1939

Kao, Yu-Chien............................ 55, 56

Kaplan, Cynthia ........................... 1883

Kapoor, Prashant ............................ 1396

Kapp, Meghan....................... 57, 1633

Karajannis, Matthias ..................... 1867

Karam, Jose A …………………..... 952

Karam, Martin............................... 1567

Karamchandani, Dipti M .... 654, 713,

Karamitopoulou, Eva .................. 1788

Karandikar, Nitin J ...................... 1503

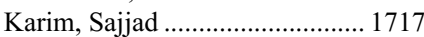

Karnes, R Jeffrey............................ 990

Karnezis, Anthony N........ 1177, 1201

Karnik, Tejashree ................... 189, 190

Karow, David .................................. 991

Karpova, Irina .............................. 1844

Karrs, Jeremiah ................................ 16

Karumanchi, SA.................................. 9

Karunamurthy, Arivarasan ............ 600

Kasago, Israel........... 132, 1881, 2162

Kashikar, Nilesh.......................... 1783

Kashiwagi, Eiji....................... 941, 951

Kashyap, Seema ........................... 1335

Kaspirkova, Jana ........................... 1178

Kassem, Maysoun ......................... 1065

Kassouf, Wassim ............................ 867

Kastenbaum, Hannah A..................... 25

Kastner, Daniel L .............................. 1358

Kasymjanova, Goulnar ............... 2005

Katabi, Nora ............. 581, 1298, 1343

Kataoka, Kensuke ....................... 1909

Katara, Rahul ................... 1752, 1991

Katava, Gordana ............................ 842

Katerji, Hani................................... 1430 
Katerji, Roula .................................. 191

Kato, Ikuma............................... 58, 71

Kato, Masashi ............................ 1067

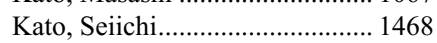

Katoh, Ryohei .................................. 610

Katsuki, Naomi ............................. 1936

Katz, Matthew H.............. 1777, 1785

Katz, Ruth L................................. 1996

Katzenberg, Jennifer ................... 1915

Kaufman, Stuart .......................... 1895

Kaufmann, Martin.............. 256, 2145

Kaur, Jaswinder............................ 1882

Kawabata, Yoshinori .................... 1943

Kawahara, Kunimitsu ................. 1942

Kawahara, Takashi ....................... 951

Kawai, Akira .................................... 98

Kawakami, Fumi.......................... 952

Kawano, Shingo .............................. 797

Kazakov, Dmitry V ......... 69, 70, 520, $521,522,984,1207$

Kazemimood, Rossana................ 1179

Keefe, Joan................................... 2059

Keenan, Sean O............................ 2050

Keeney, Matthew ............................ 553

Keeney, Michael G........................ 2084

Kefeli, Mehmet ................................ 579

Kehr, Elizabeth.............................. 1210

Keilin, Steven.................................. 756

Keiser, Elizabeth ............................. 515

Keiser, Michael ............................... 515

Keith, Christopher.......................... 995

Keller, Cesar.................................... 1944

Keller, Christian E........................ 1340

Keller, Manuel .............................. 1959

Kelley, Denise …………………..... 1393

Kelley, Todd W ................. 1457, 1477

Kellough, David A ....................... 1611

Kelly, David R ........................... 1885

Kelly, Karen ......................... 6, 8, 1907

Kem, Marina ............ 789, 1963, 2012

Kemp, Christopher....................... 1853

Kendrick, Samantha.................... 1514

Kennedy, Claire.............................. 1614

Kerl, Katrin ..................................... 521

Kern, Jason...................................... 390

Kerndt, Peter R............................ 1564

Kerr, Darcy A...... 59, 388, 400, 1221,

1328

Kerr, Kim M.................... 1915, 1947

Kerr, Sarah E.......... 1270, 1278, 2084

Kertowidjojo, Elizabeth ............. 1883

Keshavjee, Shaf............................ 1984

Ketterling, Rhett P....................... 1490

Keyhanian, Kianoosh..................... 391

Khabaz, Mohamad N ..................... 851

Khader, Samer N............................ 335

Khafateh, Youssef ....................... 1858

Khairallah, Aya............................ 1567

Khalid, Asif......................... 467, 1813

Khalifa, Mahmoud ........................ 1236

Khalifeh, Ibrahim .............. 1567, 1872

Khalighi, Mazdak......................... 1637

Khalil, Farah …………………..... 1843

Khalili, Human.............................. 1195

Khan, Farhan......................... 192, 193

Khan, Ghazal................................... 415

Khan, Khalid ................................. 1895

Khan, Saif ………………….......... 1805

Khan, Samia ....................... 72, 92, 93

Khan, Sheema ............................ 1775

Khan, Zanobia......... 1180, 1789, 1984
Khanafshar, Elham........................ 546 Khani, Francesca..... 978, 1023, 2073, 2089

Khanlari, Mahsa........ 194, 195, 1369,

Khanna, Nehal................................. 80

Khararjian, Armen H....................... 953

Khattab, Ruba ............................. 392

Khattak, Aftab ............................. 1326

Khazai, Laila ...................... 393, 2038

Khoja, Hatim................................... 494

Khoor, Andras ............................... 1944

Khor, Tze S ……………………...... 719

Khoral, Priya …………………….. 1432

Khoshkrood-Mansoori,

Babak ....................... 1940, 1987

Khoury, Joseph...... 1355, 1402, 1403, $1429,1434,1445,1468,1501$

Khoury, Joseph D

1416

Khoury, Thaer ....................... 210, 211

Khurana, Jasvir S ....... 970, 971, 972,

1327

Khurana, Kamal K ..... 422, 471, 1344

Khurana, Seema ........................... 1863

Khurana, Ujjawal ............................ 516

Khurani, Hetakshi ....................... 1762

Khurram, Muhammad................. 1094

Khurram, Muhammad S.............. 1634

Kidder, Ian J ..................................... 585

Kies, Merril ................................. 1290

Kilgore, Mark R ....... 219, 1226, 1248

Kilic, Ayse I................................... 420

Kilic, Ayse Irem ............................. 586

Kilzieh, Rania ................................ 359

Kim, Ahrong ....................... 724, 771

Kim, Amy.................................... 1688

Kim, Annette S................. 1433, 1510

Kim, Binnari ................................. 1181

Kim, Daniel J .................................... 912

Kim, Eun Kyung ......................... 1296

Kim, Eun Na ................................ 1239

Kim, Grace E .................... 1799, 2056

Kim, Haeryoung................. 1778, 1792

Kim, Hye Ryun ............................ 1296

Kim, Hyung-Gyoon ...................... 1313

Kim, Hyung-Ho .............................. 716

Kim, Hyunjae R .......................... 1903

Kim, Hyunsung ...................... 254, 255

Kim, Jaihwan ............................. 1792

Kim, James................................... 1763

Kim, Jeff....................................... 1941, 2156

Kim, Jimin...................................... 60

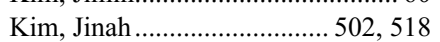

Kim, Jin-Won .............................. 1792

Kim, Jisun ........................... 1312, 1854

Kim, Jisup ................................... 1635

Kim, Ji-Yong Julie ....................... 1219

Kim, Jong........................... 1742, 2045

Kim, Joo Yeun................................. 770

Kim, Jung-sun .................................... 1181

Kim, Kyoung-Mee ....... 633, 636, 659

Kim, Kyu-Rae ................... 1109, 1239

Kim, Min-Hee ................................. 584

Kim, Riyeon Laura......................... 257

Kim, Rob.................................... 1857

Kim, Seok-Hyung .......................... 130

Kim, Seung Tae............................... 636

Kim, Sungeun ...... 132, 196, 763, 944

Kim, Sung Joo.............................. 1790

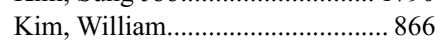

Kim, Woo Ho ................................. 716
Kim, Yeseul........................... 254, 255

Kim, Young ............ 1081, 1515, 1545 ,

2102

Kim, Young Keum ....... 724, 770, 771

Kim, Young S .................. 1518, 1571

Kim, Yourha ......................................584

Kimbrell, Hillary .............................. 518

Kimler, Bruce ................................. 190

Kimmelshue, Katherine .............. 1303

Kimura, Kiminori.......................... 1684

Kimura, Kyosuke ........................... 1067

Kimura, Takahiro ........................... 1040

Kimura, Tohru ................................ 1067

Kindblom, Lars-Gunnar.................... 34

King, Caleb ................................... 954

King, Rebecca ................... 1396, 1652

Kini, Ameet R ............................ 1476

Kini, Lata .................... 35, 1752, 1991

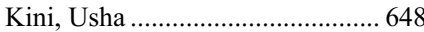

Kinloch, Mary ............................... 1218

Kinney, Marsha C ........................ 499

Kinoshita, Akira ........................... 2142

Kinoshita, Fumie............................ 1067

Kinoshita, Yoshiaki .......... 1942, 2011

Kinzler, Ken .................................. 1018

Kip, Nefize S .................................. 197

Kipp, Benjamin .................. 1808, 2010

Kiran, Sayee .................................. 292

Kirfel, Jutta ...................................... 996

Kirkwood, John M ......................... 495

Kirsch, Ilan.................................. 1493

Kirsch, Richard ..................... 672, 673

Kis, Lorand .................................. 1354

Kishimoto, Hiroshi............................... 98

Kishiwada, Masayuki.................. 1816

Kisiel, John B................................ 755

Kiss, Katalin.............. 517, 1284, 1292

Kisseleva, Tatiana ....................... 1686

Kissiedu, Juliana O ...................... 714, 715

Kitamura, Yuka ........................... 1958

Kitlinska, Joanna................................... 1874

Kito, Masahiko............................... 1182

Kiyokawa, Takako ............. 1244, 1245

Kjaer, Susanne K......................... 1271

Klampatsa, Astero ......................... 1980

Klein, Eric A........................ 903, 1085

Klein, Melissa ............................... 2077

Klein, Oliver ................................. 2157

Kleinman, George ............. 1732, 1765

Kleinschmidt-DeMasters, Bette...

1743,1753

Klevos, Geetika............................... 195

Kliger, Alan ....................................... 545

Klimek, Szczepan ........................... 860

Klimstra, David S........ 668, 732, 733, $1773,1807,1911$

Klöppel, Günter................. 1773, 1791

Kluemper, Niklas ............................ 996

Kluge, Michelle L ................. 310, 311

Kluk, Michael ............................... 1349

Klump, William................................ 434

Knickle, Corey .............................. 1638

Knight, Deborah................... 694, 746

Knox, Jennifer J ....... 638, 1789, 1835

Knudsen, Erik S ........... 100, 237, 809

Knudson, Beatrice......................... 1856

Ko, David.................................... 127, 128

Ko, Huai-Bin Mabel.... 729, 730, 731, $736,737,742,743,843,844$,

Ko, Hyang-Mi
Ko, Jennifer.......... 424, 500, 518, 519

Ko, Young Hyeh........................... 1468

Ko, Young-Hyeh ........................... 1363

Kobalka, Peter J ............................. 394

Kobayashi, Eisuke............................. 98

Kobayashi, Yukihiro........................ 758

Kobel, Martin ......................1183, 1184

Koch, Lisa ......................... 1659, 1673

Kocher, Jean-Pierre A................. 1278

Kocherginsky, Masha.......... 966, 1062

Kocovski, Linda ................................. 318

Kodira, Chinnappa ........................ 163

Koebel, Martin ......... 814, 1151, 1218

Koehne de Gonzalez, Anne......... 675, $1678,1679,1693$

Koepsell, Scott A.......................... 1197

Koga, Hironori ............................... 1684

Koh, Jiwon ....................................... 716

Koh, Valerie ...................................... 198

Koh, Xue-Qing.............................. 1196

Kohashi, Kenichi.............................97

Kohno, Takashi ................................ 98

Kojima, Motohiro ........................... 797

Koka, Madhurima ................ 258, 259

Kokuho, Nariaki............................... 1943

Kolesar, Jill M.............................. 1907

Kolin, David................................... 1185

Kollias, James .................................. 153

Kolodgie, Frank ............................ 323

Komarow, Hirsh ................................ 1536

Komissarova, Elena V.......... 675, 840

Kommoss, Friedrich..................... 1201

Kommoss, Stefan ........................... 1201

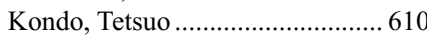

Kondoh, Yasuhiro........................... 1909

Konety, Badrinath ................. 437, 991

Kong, Beihua .................................. 1227

Kong, Christina ............................... 371

Kongkarnka, Sarawut..................... 840

Konnick, Eric Q .......................... 1066

Konopleva, Marina ...................... 1355

Konstantinova, Anastasia M ....... 520,

Konukiewitz, Björn........................ 1791

Koo, Jamie ..................................... 892

Kooby, David ............................... 1800

Koong, Albert.............................. 1803

Kopetz, Scott........................ 749, 1824

Korangy, Elizabeth M .................... 263

Kornacki, Susan ............................. 695

Korourian, Soheila ........................... 2134

Korsunskiy, Ilya ........................... 1195

Kosari, Farhad..... 923, 931, 942, 990,

1956

Kosemehmetoglu, Kemal................ 61

Koshiol, Jill ........... 1774, 1778, 1795,

1808

Kostroff, Karen M.......................... 168

Kotzer, Katrina E ................... 310, 311

Kouba, Erik .......................... 1031, 1313

Kougioumoutzakis, André .......... 2125

Kouji, Hiroyuki ............................. 1684

Koutlas, Ioannis ........................... 1286

Koutroumpakis, Efstratios .......... 1919

Kovach, Alexandra....................... 1363

Kovtun, Irina V ............................... 990

Kowalik, Artur .................................. 501

Kozak, Margaret.......................... 1803

Kraft, Adele O.......... 433, 1288, 1976

Kragel, Peter ............................. 8, 564 
Krane, Jeffrey F........... 474, 607, 614, 1310,1321

Krasinskas, Alyssa ...... 417, 432, 671, 714, 715, 756, 787, 837, 1657, $1794,1795,1799,1800$

Krasnov, George............................ 1845

Kratzke, Robert ............................. 1907

Kraus, Amanda M ........................ 717

Krausz, Thomas ....... 628, 1123, 1980

Kravtsov, Oleksandr............. 845, 955

Kreipe, Hans H............................... 1945

Krencz, Ildiko .............................. 1944

Kresak, Jesse L.......... 726, 775, 1738 ,

1742

Krigman, Hannah............. 2044, 2093

Krings, Gregor .... 116, 171, 199, 200, $261,267,1108,1829$

Kris, Mark ……............................. 1953

Krishnamurthy, Savitri........ 455, 456,

472

Krishnamurti, Uma ...... 274, 417, 432

Kristiansen, Anna.......................... 896

Krizova, Adriana .............................. 891

Kruczek, Kimberly R .................... 443

Krueger, Jo Ellen........................... 1508

Krupar, Rosemarie ...................... 1314

Krysiak, Kilannin......................... 1496

Krystel-Whittemore, Melissa..... 1186

Kryvenko, Oleksandr N ...... 475, 956, 976, 1077, 2133

Kshettry, Varun.............................. 1741

Kua, Boon .................... 884, 885, 886

Kuba, Gabriela .............................. 164

Kuba, Maria G ................................. 201, 202

Kubal, Chandrashekhar................ 795

Kuban, Joshua D .......................... 1996

Kubilis, Paul.................................. 1738

Kubo, Takashi ................................. 98

Kucuk, Omer.................................. 927

Kudaybergenova, Asel ................. 203

Kudlow, Brian ........1189, 1295, 2119,

2121

Kudose, Satoru....... 1620, 1636, 2051

Kudryavtseva, Anna......... 1844, 1845

Kuehnel, Mark ……...................... 1945

Kuerer, Henry M ................................. 472

Kuhar, Matthew ………………….... 23

Kuick, Chik Hong ........................ 1869

Kuk, Cynthia ............................... 1070

Kulac, Ibrahim ............................ 993

Kulkani, Supriya ........................ 2035

Kulkarni, Girish S ...................... 1070

Kulkarni, Kirti M ............................ 170

Kumar, Chitra................................. 2147

Kumar, Kirthi .................... 1484, 1508

Kumar, Mohit................... 1752, 1991

Kumar, Mukesh.......................... 1752

Kumar, Priyadarshini ......... 554, 1593

Kumar, Rajiv ............ 634, 1289, 1324

Kumar, Senthil R........................... 901

Kumar, Sunil ................................. 1933

Kumar, Vipin......................... 35, 1752

Kumarapeli, Asangi R................ 2134

Kumarasinghe, Priyanthi.............. 719

Kumari, Swati .................. 1339, 1802

Kumeda, Cristina .............. 1450, 1451

Kunchala, Sudhir............................ 1074

Kunder, Christian A.............. 567, 589

Kunju, Lakshmi P ...... 908, 909, 1068

Kunkalla, Kranthi........................ 1532

Kunugi, Shinobu ........................... 1943
Kuo, Frank C........... 524, 1456, 1542, $1543,1546,1847$

Kuo, Huai-Ching......... 871, 872, 912,

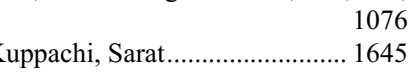

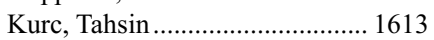

Kurman, Robert........................... 1271

Kurnit, Katherine ...............1129, 1187

Kuroda, Kishio ................................. 317

Kuroda, Naoto............................... 984

Kurosawa, Sayaka............................ 764

Kurt, Habibe................................... 1434

Kurtycz, Daniel ................................. 464

Kuruzar, Gordana .......................... 155

Kurzawa, Pawel .............................. 42

Kushida, Yoshio ............................ 1936

Kutys, Robert ................................ 323

Kuwaki, Kenji .................................. 317

Kuzon, William.............................. 151

Kvasnicka, Hans Michael .......... 1435

Kwak, Heewon........... 718, 719, 1656

Kwiatkowski, Fabrice ................... 248

Kwok-Cheung, John C................ 2133

Kwon, Hee Jung .............................. 204

Kwon, Sook-kyung ........... 1315, 1645

Kwon, Yuri ................................... 1406

Kyoko, Kurosawa ........................ 1558

Kyrpychova, Liubov ............ 521, 522

Kyrpychova, Lubov ....................... 520

Kyung, Kim Woo ........................... 1809

Labarge, Brandon........................... 612

Lacasse, Yves .............................. 1975

Lacayo, Norman..... 1437, 1505, 1506

Lackner, Karoline......................... 1671

Lacroix, Ludovic............................ 473

Lacy, Antonio .................................. 625

Ladanyi, Marc ....... 1831, 1837, 1867, 1896, 1903, 1910, 1953

Ladich, Elena R............................ 323

Ladwig, Nicholas ....................... 1188

Laenger, Florian ............................ 1945

Laffin, Jennifer J .......... 979, 980, 981

LaFramboise, William A ............. 1949

Laga, Alvaro C ........................... 1576

Lagana, Stephen M ... 723, 783, 1678,

1679,1693

Laganosky, Dean ........................... 995

Lagoo, Anand S............................. 1447

Lagstein, Amir..................... 497, 1214

LaGuardia, Lisa.............................. 706

Laguerre, Brigitte.......... 946, 947, 948

Lai, Chi K................................... 1904

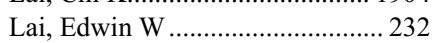

Lai, Jinping .................................... 572

Lai, Keith ....................................... 705

Lai, Victoria ................................ 1953

Lai, Win Shun ..................... 878, 918

Lai, Yue-Yun .............................. 1538

Laklouk, Israa ................................ 395

Lal, Priti ......................319, 709, 972

Lalonde, Amy........................ 623, 805

Lam, Lucia LC ............................. 1056

Lamaison, Claire ......................... 1444

Lamarca, Angela ............................ 762

Lambert, James R........................... 955

Lambert, Pascal........................... 1483

Lambertz, Allyn M......................... 1315

Lam-Himlin, Dora M ......... 806, 1786

Lammert, Craig ............................. 1694

Lamps, Laura ................................. 698

Lamy de la Chapelle, Thierry .... 1444
Lancet, Jeffrey E ........................... 1553

Landau, Carrie.............................. 1050

Landau, Michael .................. 720, 745

Landgraf, Ralf............................. 1532

Lange, Jane ................................... 1066

Langer, Rupert ............................... 1959

Lang Kuhs, Krystle ....................... 1323

Langone, Anthony........................ 1633

Langstraat, Carrie L ..................... 1270

Lanigan, Christopher.......... 122, 1655

Lanjewar, Sonali ................. 583, 1436

Lapadat, Razvan...... 957, 1594, 2048,

2081

Lapinski, James.............................. 705

Larios, Jose M.

123

Larocca, Luigi M ................... 439, 490

La Rosa, Stefano ................. 570, 1782

Larque, Ana B ...

..... 62

Larson, Alexandra ........................... 289

Larson, Brent................................... 830

Laser, Alice ................................. 2077

Laskar, Derek B .......................... 2052

Laskar, Siddhartha ............................ 80

Laskin, Janessa............................ 1213

Laskin, William B

..... 64

Lasota, Jerzy ......................... 501, 702

Lastra, Ricardo R ........ 338, 354, 415 , $416,483,1157,1254,1255$

Laszik, Zoltan ........ 1663, 1829, 2056

Latchminsing, Kerri Ann .............. 741

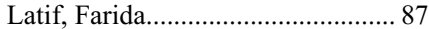

Lau, Hubert ........................................ 396

Lau, Kowk Fai .............................. 236

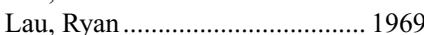

Lau, Ryan P......................... 343, 397

Laucirica, Rodolfo ......................... 481

Laughlin, Todd .................... 1385, 2120

Laurent, Camille .......................... 1390

Laurent-Puig, Pierre ……………..... 779

Lauwers, Gregory E.......... 698, 1809 ,

2056

Lauwers, Gregory Y....................... 662

LaViolette, Peter S ...................... 2131

Law, Charles ............................... 1624

Law, Mark E................................. 1554

Lawrence, W Dwayne...... 1098, 1099 , $1205,1206,1273$

Lawrenson, Kate ............... 1260, 1261

Lawson, Barrett................... 413, 1203

Lawson, Diane ......... 632, 932, 1299 , $1575,1657,1964,2143$

Layden, Brian............................. 1704

Layfield, Lester .......................... 901, 958, 2053

Lazar, Alex ..................................... 522

Lazar, Alexander J....... 32, 41, 45, 72, $92,93,94,533,537,1758$

Lazar, Vladimir

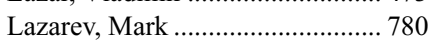

Lazaryan, Aleksandr ................... 1502

Lazenby, Audrey J......................... 1582

Laziuk, Katsiaryna ............ 1371, 2053

Lazzareschi, Daniel...................... 1437

Le, Long ........................................ 438

Leach, Patrick .............................. 1930

LeBeau, Shane O ........................... 425

Leblond, Frédéric .............. 2125, 2152

LeBoit, Philip .................................. 511

Lebwohl, Benjamin.......................... 723

Lechpammer, Mirna .................... 1757

Lecumberri, Arturo ....................... 1739

LeDoux, Shawn

2031
Leduc, Charles ............................... 2003

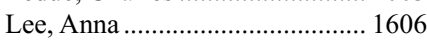

Lee, Annette ................................. 1195

Lee, Bernett........ 184, 185, 304, 1840

Lee, Boram...................................... 131

Lee, Byung-Heon .............................. 204

Lee, Chang Hun ..................... 724, 771

Lee, Cheng-Han .......... 40, 814, 1126,

1189,1295

Lee, Cheryl T 1049

Lee, Chi Hyun............................. 1193

Lee, Chung-Han ................................ 874

Lee, Chung-Ta................................... 721

Lee, Cleo ………………………... 1039

Lee, Hee Eun.... 721, 722, 754, 1946, 2008, 2009, 2010

Lee, Ho-chang .............................. 205

Lee, Hwajeong ........... 726, 763, 1654,

1881,2162

Lee, Hyejung................................. 1792

Lee, Hye Seung .............................. 716

Lee, Jack ..................................... 1824

Lee, James.......................................... 402

Lee, Jeeyun .................................... 636

Lee, Jeffery E ................................. 1785

Lee, Jeffrey.................................. 1777

Lee, Jen-Chieh .............................. 1189

Lee, Jennifer LF .......................... 1526

Lee, Jerome E................................ 2119

Lee, John P.............. 588, 1438, 1884

Lee, June G …………………….... 883

Lee, Kenneth K ............................. 1813

Lee, Keun-Wook ............................. 716

Lee, Kyoung Bun .......................... 1778

Lee, Lames....................................... 390

Lee, Li-yu........................................ 1579

Lee, Matthew …………………..... 1305

Lee, Michael .............. 527, 723, 1679

Lee, Michelle ................................. 236

Lee, Minji........................................ 280

Lee, Ok-Jun...................................... 205

Lee, Paul ..................................... 1190

Lee, Peng....... 856, 1000, 1072, 1073 ,

1089,1090

Lee, Sandra

1183,1191

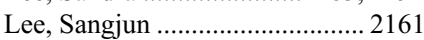

Lee, Sangkyou................................... 883

Lee, Seung J ........................................ 44

Lee, Seungjae................................. 495

Lee, So Jeong ............... 724, 770, 771

Lee, Thomas D............................... 2054

Lee, Thomas K............................... 2065

Lee, Victor K ....................................... 63

Lee, Winston ................................... 1439

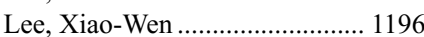

Lee, Yangkyu ................................ 1792

Lee, Yi-Chien ................................ 1874

Leeming, Rosemary ...................... 197

Lefaivre, Michaela ......................... 1701

Lefort, Mathilde ……..................... 947, 948

LeGallo, Robin............................. 1876

Legesse, Teklu................................. 398

Leggett, Barbara............................. 728

Lehman, Norman ......................... 1611

Lehmann, Brian.............................. 164

Lehrer, Jonathan ............................. 930

Lehrke, Heidi ................................... 1680, 2084

Lei, Li............................................... 17

Leibovich, Bradley C ........................ 922

Leich, Ellen .................................. 1380

Leite, Katia RM

$.399,959$ 
Leith, Kate....................................... 122 Leivo, Mariah Z ......... 960, 991, 1051 Lele, Subodh M............................ 1197 Le Malicot, Karine .......................... 779 Lemos, Maria M.............................. 367 Lennerz, Jochen K ............ 1585, 2101 Lennon, Anne Marie ...................... 761 Leone, Dominick............................... 503 Leone, Jose P...................... 209, 1103 Leone, Lisa........................................ 18 Leone, Pamela.................................. 706

Leong, May Ying ........................... 167

Lepe, Marcos.................................... 206

LePhong, Christopher D ............. 2055

Lerner, Seth P................................ 976

Leslie, LSuzanne .............................. 230

Lesluyes, Tom ............................... 1128

Lespagnol, Alexandra ......... 946, 947,

Lessi, Francesca ............................... 66

Le Stang, Nolwenn............................. 1980

Lester, Susan C ..................... 201, 202

Leteurtre, Emmanuelle................... 601

Leung, Cheuk Hong ......................... 94

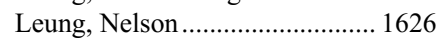

Leung, Samuel ............................ 1177

Leventaki, Vasiliki ............ 1354, 1492

Levi, Angelique............................... 940

Levine, Pascale ............................... 352

Levinson, Howard......................... 526

Levinson, Shauna... 1678, 1679, 1693

Levy, David................................... 589

Lew, Madelyn ....................... 401, 868

Lewen, Margaret ........................... 1544

Lewis, James ......... 1282, 1292, 1303,

$1316,1323,1346$

Lewis, Jenna.......................... 388, 400

Lewis, Melinda .............................. 372

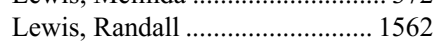

Lewis, Robert............................... 2124

Lheureux, Stéphanie .........1137, 1185

Li, Anna Fen-Yau .................. 823, 824

Li, Anqi ........................................... 207

Li, Arthur........................................ 1081

Li, Chien-Feng ............................. 929

Li, Dan .......................................... 1825

Li, Dehong ................................... 150

Li, Faqian ................... 449, 606, 1236

Li, Geling ...................................... 1885

Li, Haocheng................................ 1449

Li, Haonan............................ 635, 674

Li, Hongjie .................................... 725

Li, Hui ......................................... 1440

Li, HuiHua ................... 129, 134, 167

Li, Jia......................................... 1862

Li, Jie........................................... 1544

Li, Jingyi ................ 1402, 1500, 1549

Li, K David .................................. 1457

$\mathrm{Li}, \mathrm{Li}$.............................. 1227, 1440

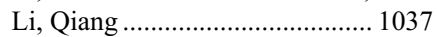

Li, Qing K .............. 1850, 1948, 1979

Li, Shaoying ........... 1352, 1402, 1416,

1500,1549

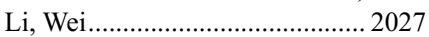

Li, Weihong....................................... 401

Li, Xiaodun ...................................... 1834

Li, Xiaoqiu ......................................... 1862

Li, Xiaoxian ........ 177, 208, 216, 217 ,

$218,228,229,274$

Li, Xin....... 141, 209, 357, 835, 1103 ,
Li, Xinyan

1595

Li, Yanchun .......................... 210, 211

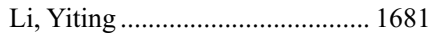

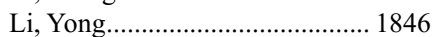

Li, Yuan........................... 1370, 1990

Li, Yunjie........................................... 961

Li, Yuping..................................... 1081

Li, Zaibo.... 179, 180, 212, 213, 1132,

1192,1610

Li, Zhongze .................................... 531

Lian, Derrick ..................................... 1869

Lian, Fei .......................................... 995

Liang, Li....................962, 1193, 1194

Liang, Sharon X ................. 800, 1195

Liang, Sheng-Ben ...................... 1835

Liang, Wen-Yih .................... 823, 824

Liang, Yaoming............................ 1269

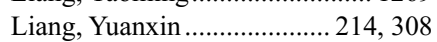

Liao, Jie................................ 635, 674

Liao, John.................................... 1138

Liao, Xiaoyan.............................. 1947

Liao, Zhiming ................................. 1039

Libe, Rossella.................................. 601

Lichner, Zsuzsanna ......................... 963

Lichtman, Andrew H....................... 315

Lidke, Diane S .......................... 1516

Lieberman, Richard...................... 2086

Ligato, Saverio .............................. 660

Ligon, Azra H.................... 1720, 1745

Ligon, Keith L...................... 1720, 1745

Lilo, Mohammed T ..... 374, 402, 403,

436

Lim, Beom Jin............................. 1635

Lim, Bora ........................................ 147

Lim, Diana ................................... 1196

Lim, Huey J........................................ 63

Lim, Jeffrey Chun Tatt ................... 184

Lim, Jeffrey CT.......... 185, 304, 1840

Lim, JingQuan.............................. 278

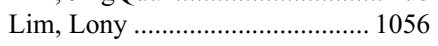

Lim, Megan S.............................. 2047

Lim, Swee Ho ................................ 167

Lim-Dunham, Jennifer................... 586

Lin, Chieh-Yu..... 271, 567, 589, 1106

Lin, Diana ................................... 1308

Lin, Douglas................................. 1173

Lin, Fan ...... 429, 463, 656, 964, 965, $1682,1711,1806,1814,2156$

Lin, Grace Y..... 650, 727, 1915, 1947

Lin, Heather

93,94

Lin, Henry.

.. 1565

Lin, Jingmei ............... 657, 726, 1683

Lin, Jonathan H....... 1051, 1749, 1754

Lin, Leo.......................................... 727

Lin, Ming-Tseh ............................... 1555

Lin, Oscar............................. 404, 447

Lin, Pei ........................... 1352, 1500

Lin, Xiaoqi .............................. 64, 383

Lindberg, Guy .............................. 1583

Lindberg, Johan.............................. 895

Lindeman, Neal I ........ 710, 785, 833, $1162,1163,1212,1720,1847$

Lindsey, Kathryn G...................... 954

Linehan, Marston W ...................... 989

Linehan, WMarston ......................... 982

Lingen, Mark................................. 1286

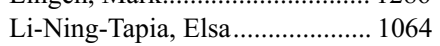

Linos, Eleni ....................................... 515

Linos, Konstantinos ……………... 523

Lintel, Nicholas................ 1197, 1582

Lionakis, Michail .......................... 626
Lis, Rosina ........................... 873, 987

Lisovsky, Mikhail ......................... 710

Litvak, Anna M ........................... 1911

Litzenburger, Beate...................... 1824

Litzky, Leslie............................... 1980

Liu, Bei ........................................... 1511

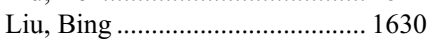

Liu, Catherine L .................... 531, 532

Liu, Chang........................................ 498

Liu, Chen.................................... 1675

Liu, Cheng............................. 343, 728

Liu, Eric ................................................... 686

Liu, Fangfang .................................. 1711

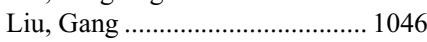

Liu, Haiping .................................... 215

Liu, Haiyan ......... 429, 463, 964, 965,

$1682,1806,1814$

Liu, Huifei ....................................... 1441

Liu, Huiping ................................... 160

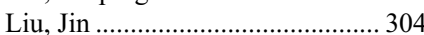

Liu, Jonathan TC.... 1014, 2080, 2137

Liu, Kai-Yan......................................... 1538

Liu, Lifang.....

1981

Liu, Lina. 102,1039

Liu, Qiang .............. 1839, 2016, 2074

Liu, Qingqing ................ 729, 730, 731

Liu, Sandy .................. 668, 732, 733

Liu, Shiguang ............................... 312

Liu, Su-Yang ................................ 2056

Liu, Ta-Chiang ........................ 735, 1695

Liu, Tzu-Ying............................... 1049

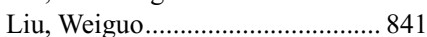

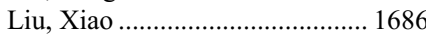

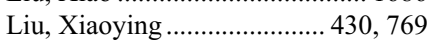

Liu, Xin ........................... 1442, 1447

Liu, Xiuli............. 409, 654, 726, 1674

Liu, Yan-Rong............................... 1538

Liu, Yanwei ....................................... 565

Liu, Yen-Chun ................................. 1443

Liu, Ying ……………………......... 571

Liu, Yongjun.................................... 2057, 2058

Liu, Yuan..... 177, 216, 217, 218, 228 ,

229,252

Liu, Yuxin..................485, 1112, 1198

Liu, Zach ............................................... 860

LiVolsi, Virginia A ............................. 582, 598

Ljung, Britt-Marie............................ 546

Llamas Gutierrez, Francisco ....... 1444

Llobeta-Navas, David .................. 1229

Llombart-Bosch, Antonio ........... 1338

Llovet, Patricia

1685

Lloyd, Isaac E ………......... 405, 1637

Lloyd, Ricardo V............... 573, 574, 575, $582,591,592,602,605,608$, $618,619,2133$

Lo, Amanda E .............................. 1886

Lo, Bryan ........................................... 1793

Lo, Raymundo W ............................. 276

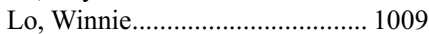

Lo, Yungtai.......................................... 1839

Loayza-Vega, Kevin...................... 2106

Lobo, Nazleen C ……................ 466

Locatelli, Francesca ......................... 1199

Lockley, Michelle ......................... 1151

Loda, Massimo............... 873, 897, 987

Logan, Suzanna J ........ 216, 217, 218,

274

Loghavi, Sanam .... 1355, 1403, 1416, $1429,1445,1501$

Logunova, Valentina .................... 1810

Loh, Amos

1869
Loh, Eva......................................... 1869

Lombardi, Celestino P.................. 439

Lombardo, Kara A....... 540, 700, 747, 777, 831, 917, 939, 977, 1917,

2135

Lonardo, Fulvio............................ 1897

Lone, Waseem .................................... 1380

Long, Hanan..................................... 835

Long, Steven ........................ 371, 396

Long, Theresa................................958

Long, Thomas .................................. 966

Long, Thomas H ……................. 219

Longacre, Teri ....371, 676, 688, 1119 ,

1120,1124

Longatti, Pierluigi ........................ 1750

Lopategui, Jean ............................... 734

Lopes, Beatriz …………………... 1753

Lopez, Jose I ................................. 1065

Lopez, Rocio ………............ 705, 829

López, Rocio P............................. 587

Lopez, Tilcia ................................ 967

Lopez-Corella, Eduardo ................... 26

Lopez-Guillermo, Armando........ 1409

Lopez-Hidalgo, Juliana E............ 1446

Lopez-Hisijos, Nicolas....... 968, 2020

Lopez Janeiro, Alvaro .................. 1739

Lopez-Ridaura, Ruy......................... 26

Lopez-Santamaria, Manuel ......... 1875

Lordello, Leonardo ....................... 1094

Lorentz, Adam................................ 995

Lorenzetto, Erica................................ 81

Lorenzoni, Cesaltina .................... 1573

Losada, Daniele M....... 862, 863, 864

Losada, Hector ........ 784, 1774, 1804,

1808

Lotan, Tamara L.......... 124, 282, 751 , $859,860,930,1056,1057$

Louis, David N............................. 1753

Louissaint, Abner ................................. 1525

Lovane, Lucilia ............................. 1573

Lowenthal, Brett M....................... 969

Lowy, Andrew M ........................... 818

Lozada, John R............................... 220

$\mathrm{Lu}$, Cheng........................................ 1316

Lu, Chuanyi Mark .......................... 1886

Lu, Chuanyong...................................... 65

Lu, Dan ......................... 406, 407, 485

Lu, Fang-I .........231, 298, 1160, 1161

Lu, Guolan ...................................... 998

Lu, Haiyan.............................. 408, 409

Lu, Miao........................................... 1638

Lu, Pin ........................................... 1862

Lu, Shaohua ................................... 1903

Lu, Shaolei .............. 1916, 1917, 2135

Lu, Xiamon ...................................... 505

Lu, Xiaomei .................................. 1445

Lu, Xinyan ............. 1352, 1922, 2040

Lu, Zhichun........................... 221, 410

Luan, Lan ..................................... 1707

Luc, Jessica GY............................... 1914

Lucas, David R................................. 590

Lucas, Elena................ 265, 423, 2049

Lucia, M Scott................................. 955

Luconi, Michaela ............................ 596

Luebker, Stephen A...................... 1197

Luedke, Catherine .............. 1447, 1886

Luffman, Christina I........... 1624, 1649

Lugli, Alessandro ....... 672, 673, 1788

Lui, Weng-Onn............................... 498

Luinetti, Ombretta................................ 819

Luketich, James D......................... 642 
Lum, Trina.......................... 1330, 1333

Luna, Eric...................... 370, 380, 440

Luna, Isabella ................................ 380

Lundberg, George D ........................ 23

Lunde, John H.............................. 1413

Luo, Wenyi........................................ 1448

Luo, Xunda ........ 970, 971, 972, 1327

Luo, Yuling............. 1682, 2116, 2156

Luong, Winnie................................. 544

Luskin, Marlise ........................... 1544

Luthra, Raja........... 1488, 1539, 1846,

1922,2040

Luthra, Rajyalakshmi.......... 147, 537,

749,1721

Luthringer, Daniel J ....................... 892

Ly, Amy........................................ 2060

Ly, Charles J................................. 1819

Lynch, Edward ....................... 376, 926

Lyons, Lisa ...................................... 21

Ma, Changqing............................... 735

Ma, Deqin ............................. 54, 384

Ma, Jiao....................................... 1463

Ma, Julia.......................................... 1039

Ma, Junsheng .............................. 472

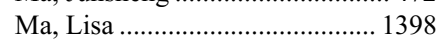

Ma, Sophia .................................. 1200

Ma, Xiao-Jun......... 1682, 1941, 2116,

2156

Ma, Yihong R....................... 736, 737

MacConaill, Laura E.........1162, 1163,

1212

MacDonald, Scott ........................... 466

Macedo, Gabriel S ......................... 161

MacGrogan, Gaëtan ..................... 1128

Machado, Isidro ............................ 1338

Machado, Rosalie.......................... 582

Macher, Bruce ................................ 1865

Macias, Virgilia ................. 1838, 2104

Mack, Philip................................ 1907

MacKay, Helen ............................. 1185

Mackers, Paula ............................... 359

Mackey-Bojack, Shannon M .......... 21

Mackinnon, Alexander C .......... 1672,

1770, 1997

Mackrides, Nicholas .................... 973

MacLennan, Gregory T.... 1016, 1091

Macon, William R....................... 1490

Macrae, Finlay ...................... 791, 792

MacRae, Robert M...................... 1907

Madabhushi, Anant ........... 187, 1316, $1603,1607,1864,2141$

Madan, Rashna..................... 189, 431

Madarnas, Yolanda......................... 257

Madduri, Deepu ............................. 1377

Madrid, Manuelito A....................... 276

Madrigal, Emilio ......... 555, 557, 562,

1596,1598

Maeda, Daichi .........1156, 1182, 1249

Maedler, Chelsea............................. 738

Maestro, Roberta................................ 81

Maetani, Iruru …………................ 1796

Magee, Fergall ............................. 2091

Magee Owens, Jordan................. 2146

Magers, Martin J ................ 590, 1597

Magi-Galluzzi, Cristina....... 848, 849, $903,1065,1085$

Maglantay, Remegio J...... 2059, 2060

Magliocca, Kelly ........................... 1292

Magliocca, Kelly R ...................... 1304

Magliocco, Anthony..... 50, 603, 1843

Magrill, Jamie .............................. 1201
Maguire, Aoife …............................ 220

Maguire, John A .............................. 10

Mahadevan, Navin ....................... 524

Mahadevan-Jansen, Anita ............. 687

Mahajan, Akanksha...................... 1874

Mahajan, Aparna ........... 346, 377, 556

Mahalingam, Meera ..................... 503

Mahdi, Zaid........ 739, 740, 741, 1092

Mahon, Brett .............................. 1283

Mahoney, Douglas W.................... 755

Mai, Kien T .................................... 1202

Maibach, Rudolf ........................... 1875

Maisonneuve, Patrick..................... 594

Maithel, Shishir........ 715, 756, 1799, 1800,1804

Maitra, Anirban ........................... 1777

Majewski, Tadeusz........................ 883

Mak, Julie..................................... 171, 1176

Makker, Vicky ............................. 1144

Makohon-Moore, Alvin P .......... 1861

Maleki, Zahra...... 336, 403, 411, 426,

Malenie, Renuka ............................... 18

Maleszewski, Joseph J ........ 309, 310, 311, 1671, 1971, 1999, 2084

Malik, Aatika......................... 336, 411

Malik, Faizan .............................. 1283

Malik, Prabhat........................... 1933

Mallick, Indranil ............................ 794

Malm, Ian-James............................ 1302

Malone, Carmel.............................. 120

Malone, Greg ................................ 884, 885, 886

Malpica, Anais .......1093, 1136, 1139,

1140

Mamone, Linda .............................. 312

Manam, Monica ........................... 1720

Mancini, Irene .................................... 46

Mancini, Massimiliano ..................... 66

Mancini, Maureen G ............................ 114

Mancini, Michael A........................ 114

Mandal, Rakesh................... 591, 1495

Mandell, James W....................... 2138

Mandolesi, Alessandra ..................... 81

Mandt, Randal L ............................ 1601

Manek, Roxanne ......................... 1260

Mangray, Shamlal ..................... $272,977,1891$

Mani, Malary.... 557, 743, 2061, 2062

Maniar, Kruti P....... 1219, 1256, 2036

Mankaney, Gautam ........................ 706

Mann, Steven A.................. 974, 1683

Mannan, Abul Ala Syed Rifat ..... 555, 557, 695, 742, 743, 1317, 1590, $1598,1801,2061,2062$

Manne, Upender..........

1313

Mannelli, Massimo .......................... 596

Mannermaa, Arto .......................... 207

Mannu, Claudia ............................. 1486

Manoj, Namitha ............................ 2119

Manon, Luis .................................. 2148

Mansfield, Aaron S... 535, 1956, 1998

Mansoor, Mohammad O ............. 1449

Mansoor, Wasat.............................. 1961

Mansukhani, Mahesh M... 1478, 1489

Mantilla, Jose G ............................. 975

Mantovani, Giovanna.......................... 47

Manucha, Varsha......................... 2024

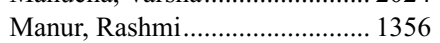

Manzoni, Marco ............................ 2136

Mao, Tsui-Lien................................ 350

Mao, Zhengwei J............................. 1529

Marbury, David C ......................... 412
Marchand, Vinciane ...................... 1973

Marchant, Kandice...................... 2050

Marchesi, Raquel ............. 1450, 1451

Marchevsky, Alberto M .... 568, 1608,

Marchio, Caterina ...................... 1152

Marco, Rex......................................... 36

Marco, Vicente ..................... 222, 223

Marcogliese, Andrea N ............... 1379

Marcus, Elizabeth A ...................... 1713

Mardekian, Stacey K......................... 1318

Marder, Galina S ............................. 293

Maree-Haynes, Ann ...................... 1603

Marey, Karima I ............................ 1751

Marginean, Celia............... 1677, 1793

Margolskee, Elizabeth.................. 1452

Marguet, Florent.......................... 1972

Margulies, Kenneth....................... 2141

Mariani, Rachel............................. 1453

Maric, Dragan ............................... 1530

Maric, Irina ............ 1389, 1530, 1536

Marino, Danielle ........................... 622

Marino-Enriquez, Adrian ............ 1847

Maris, Alexander............................. 744

Mark, Eugene .............................. $22,1935,1992$

Marker, Daniel F ............................ 745

Markow, Michael .............................. 746

Markowitz, Arnold......................... 732

Maron, Steven .............................. 1254

Marrero Rolón, Rebecca M......... 1848

Marsh, J Wallis............................. 1813

Marshall, Audrey.......................... 1757

Marshall, James.............................. 875

Marti, Carles ………………............ 359

Marti, Lola ................................... 1229

Martignoni, Guido.......................... 869

Martin, Amanda K ......................... 2095

Martin, Ben ................. 884, 885, 886

Martin, Blanca................................ 511

Martin, Cara M........ 435, 1828, 1860

Martin, Cheyenne......................... 1516

Martin, Tina..................................... 159

Martinek, Petr ...................... 520, 522

Martinez, Anthony ............. 558, 1228

Martínez, Antonio ............... 183, 1534

Martinez, Miguel J .......................... 1573

Martinez-Cardus, Anna .................. 757

Martinez-Outschoorn, Ubaldo ... 1952

Martínez-Pozo, Antonio............... 1348

Martínez-Ricarte, Francisco....... 1748

Martínez-Sáez, Elena ................... 1748

Martin-Guerrero, Idoia................ 1409

Martini, Maurizio .............................. 439

Martino, Michele............................. 819

Marton, Elisabetta ........................... 1750

Marty, Virginie ............................... 473

Marusic, Zlatko ............................... 519

Maruyama, Yasuhiro ....................... 758

Marwah, Annika............................ 516

Maryamchik, Elena .............................. 1454

Marzullo, Andrea ......................... 1970

Masand, Ramya........... 413, 421, 460,

1203
904,1732

Masia, Ricard ............................ 22, 320

Masih, Aneal ................................ 1363

Mason, Emily F............................. 1455, 1456

Masoudi, Hamid............................... 10

Massoll, Nicole …….......................... 2134

Master, Stephen................................. 1544

Master, Viraj A ........... 927, 995, 1622
Mata, Douglas A.................... 559, 976

Mate, Jose L ……………………. 1475

Mateos, Maria E........................... 1875

Matharoo-Ball, Balwir ..................... 518

Mathew, Ashley.............................. 493

Mathew, Bipin ................................. 528

Mathew, Susan .............................. 1464

Mathews, Stephanie P................ 1481

Mathieu, Romain.......... 946, 947, 948

Matias-Guiu, Xavier .........1113, 1229

Matoso, Andres .... 747, 777, 939, 977

Matrai, Cathleen E ... 978, 1204, 1887

Matsioka, Makoto ……..................... 807

Matsko, Jonee …………………..... 357

Matson, Daniel..... 592, 979, 980, 981

Matsubara, Osamu ...................... 1935

Matsuda, Katsuya.......................... 2142

Matsumoto, Cal............................... 1895

Matsumoto, Masanori .................. 1632

Matsumoto, Shinji............ 1942, 2011

Matsumoto, Takashi .................... 1755

Matsumura, Mai........................... 2002

Matsunaga, Toru........................... 1936

Matsuno, Yoshihiro ……………... 1817

Matsuo, Kosuke ………………….... 58

Mattia, Anthony ……….................. 698

Mattis, Aras N .............. 661, 748, 817

Mattis, Daiva.................................. 748

Matushek, Scott............................. 718

Matynia, Anna P............................... 1457

Maurel, Joan.................................. 2139

Mauzo, Shakuntala......................... 525

Maxwell, Aaron WP..................... 2135

Maxwell, GLarry.......................... 1261

May, Andrea .......................................... 679

May, Gary ...................................... 458

May, Michael .................................. 1605

May, Rebecca ............................... 2063

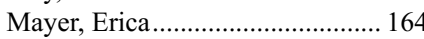

Mayer, Ingrid A ............................... 164

Mazzaferro, Vincenzo ........... 594, 595

Mazzanti, Chiara ................................. 66

McAfee, John L.................................. 1744

McAlpine, Jessica N ...........1113, 1177

McBrien, Sarah .............................. 565

McCabe, Marshall............................ 1694

McCall, Chad ............................... 1447

McCall, Shannon...... 693, 1043, 1701

McCarthy, Caitlin................... 148, 149

McCarthy, Christine ................... 1519

McCarthy, Whitney A ......... 360, 1205 ,

1206

McCarty, Erin......... 1014, 2080, 2137

McCauley, Kathleen....................... 461

McCluggage, W Glenn ....1162, 1163,

1183, 1189, 1251, 1253

McClune, Brian........................... 1502

McConechy, Melissa.....................1113

McConkey, David ............... 883, 1060

McCormick-Baw, Clare ..... 102, 2041 ,

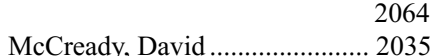

McCroskey, Zulfia............................. 224

McCuiston, Austin ............ 1319, 1948

McDaniel, Andrew S.................... 1069

McDonald, Kelsey ............................593

McDonald, Timothy M ................... 2065

McDonald, William........................ 593

McFarland, Marie ........................... 1163

McFarlane, Taneisha .................... 1320

McGarry, Sean

2131 
McGinn, John................................ 1342 McGough, Richard L ....................... 78 McGrath, Kevin ................. 467, 1813 McGregor, Stephanie M............. 1123 McGregor, Thomas B.................. 1638 McHenry, Austin .......................... 1826 McHugh, Jonathan ....... 497, 590, 726 McHugh, Kelsey .................. 414, 424 McIntire, Patrick J......... 77, 136, 137, $225,226,227$

McIntyre, John B ........................ 1183

McIver, Bryan .................................. 603

McKay, James D ................. 846, 1821

McKee, Trevor ............................ 1137

McKelvie, Penelope................... 1363

McKelvie, Penny .......................... 1513

McKenna, Barbara J...................... 726

McKenney, Jesse K..... 500, 881, 903, 1012, 1050, 1085, 1955

McKenzie, Gordon AG ............... 1320

McKinnon, Elizabeth ..................... 526

McKittrick, Ian................. 2119, 2121

McLachlin, C Meg....................... 2091

McLachlin, Meg M ............1153, 1154

McLaughlin, Ray .......................... 120

McMahon, Loralee... 622, 1075, 2095

McManus, Bruce M ...................... 316

McMasters, Richard .................... 1892

McMeeken, Emily........................... 392

McNary, Terra J........................... 1857

McNeill, Helen................................ 155

McNulty, Samantha...................... 1190

McPhail, Ellen D.... 1396, 1490, 1491

Mecca, Lauren................................ 19

Medane, Sarah................... 946, 1444

Medeiros, Bruno .......................... 1471

Medeiros, Filomena ...................... 1980

Medeiros, LJ ........... 749, 1428, 1446, 1521

Medeiros, L Jeffrey .......... 1352, 1353, $1354,1355,1357,1373,1374$, $1402,1403,1407,1408,1416$, $1417,1418,1422,1423,1429$, $1434,1441,1445,1485,1488$, $1500,1501,1539,1549,1556$,

1922,2040

Mederake, Moritz........................ 1507

Medina, Karie ............................... 1774

Meeker, Alan......... 93, 281, 282, 853,

1363

Megna, Daniel.................................. 678

Mehr, Chelsea ............................... 560

Mehra, Rohit ....... 497, 590, 868, 908 , 909, 1038, 1049, 1050, 1068, 1069

Mehrad, Mitra .......... 477, 569, 1323, 1949

Mehrotra, Meenakshi ........... 513, 749

Mehrotra, Swati.......... 351, 445, 586, 1578,1600

Mehta, Arjun ...................................... 67

Mei, Lily ........................................... 415

Mei, Zhen W ................................. 416

Meignin, Véronique ..................... 1950

Meis, Jeanne M ................................. 34

Meisel, Jane L ............................... 208

Meisner, Angela .................................... 1293

Mejias, Luis.................................. 1739

Melamed, Jonathan ..... 695, 856, 920, $1000,1072,1073,1089,1090$,

1969
Melchior, Linea C

1284

Melstrom, Laleh.....

492

Memis, Bahar............. 382, 417, 432, $671,714,756,784,787,837$, 1778, 1794, 1795, 1799, 1800,

1804,1808

Menard, Chantalle........................ 1483

Mendez, Inmaculada ..................... 223

Mendiola, Marta.......................... 1875

Mendoza, Pia........ 177, 218, 228, 229

Mendoza, Tania ............................... 750

Mendoza-Cervantes, Diana........ 1191

Mendoza Perez, Julia ....... 1002, 1047

Menendez, Clara ........................... 1573

Menes, Manuel............................. 1568

Menezes, Diana............................. 1952

Meng, Qing H ............................. 2021

Mengel, Michael .......................... 1914

Menke, Joshua.............................. 1727

Menon, Madhu P............. 1426, 1525

Mentzel, Thomas............................. 517

Mentzer, Steven J......................... 1932

Mercado, Cecilia L........................ 305

Mercan, Ezgi ................................ 2032

Mercurio, Stephanie...................... 1006

Meredith, David M............................ 68

Meric-Bernstam, Funda ................ 147

Merino, Maria J..................... 982, 989

Merzianu, Mihai.......................... 1332

Mesa, Tania ..................................... 1230

Mescoli, Claudia ................. 819, 1685

Mes-Masson, Anne-Marie.............. 988

Messer, Jamie................................. 855

Messina, Jane ............................... 1230

Meszoely, Ingrid ............................ 164

Metcalfe, Dean D........................ 1536

Mete, Ozgur ............... 579, 582, 1008

Mettler, Tetyana N...... 418, 484, 2076

Meurice, Guillaume ...................... 473

Meves, Alexander .......................... 506

Meyer, Rosana D............................ 832

Mezhir, James J............................ 1781

Mhawech-Fauceglia, Paulette... 1141,

1260,1261

Mian, Badar.... 994, 1005, 1015, 1654

Micale, Mark A .......................... 2079

Miceli, Rosalba ............................... 595

Michael, Claire W ......................... 385

Michal, Michael ........69, 70, 75, 984,

1207

Michal, Michal..... 7, 69, 70, 75, 520, $521,522,849,983,984,1054$,

1178,1207

Michalova, Kvetoslava ... 69, 70, 849, 983, 984, 1054, 1207

Michalowski, Susan M............... 1525

Michelakos, Theodoros................ 631

Middha, Sumit............ 668, 916, 1144

Middleton, Lavinia P.................. 1728

Miesbauerova, Marketa............... 1207

Miettinen, Markku M.......... 501, 702,

1338,1741

Mihm, Martin C ......................... 2133

Miki, Yasuhiro.............................. 1982

Mikita, Geoffrey........................... 1452

Miles, Rodney ............................ 1520

Milhem, Mohammed.................. 53, 89

Milikowski, Clara ........................ 1783

Milione, Massimo ................. 594, 595

Miller, Benjamin ....................... 53, 89

Miller, Caitlyn ............................... 1951
Miller, Catherine ............................ 518

Miller, Daniel L................ 1948, 2066

Miller, David ................................. 1049

Miller, Dianne ............................... 1177

Miller, Gregory ………………........ 793

Miller, James A ......... 751, 781, 1059

1127

Miller, Karin................................. 1192

Miller, Lance D ............................ 2092

Miller, Michael B ......................... 1745

Miller, Naomi ................................ 2035

Miller, Ross A .......... 224, 1912, 1913

Miller, Vincent .... 196, 238, 496, 790, 994, 1005, 1015, 1297, 1331, $1654,1836,1855,1880,1920$

1924

Mills, Anne...... 146, 539, 1155, 1208 , $1209,1228,1241,1252,1263$,

$1276,1876,2138$

Mills, Stacey E................. 1282, 2138

Milne, Katy .................................. 1787

Milner, Danny A.................. 232, 1577

Milton, Denai .................................... 525

Minami, Yuko............................... 1993

Miner, Thomas J.............................. 777

Minerowicz, Christine................. 1951

Ming, Mei ...................................... 1862

Mino, Barbara ........... 125, 301, 1193,

1194,2153

Mino-Kenudson, Mari....... 789, 1963, 1977, 1992, 1993, 2012

Mira, Beatriz ................................... 249

Miraflor, Allen............................ 1599

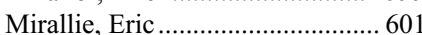

Miranda, Roberto N ........ 1408, 1428,

1500

Mirkovic, Jelena.....1162, 1163, 1210, $1211,1212,1243,1247$

Mirsadraei, Leili........... 935, 960, 985

Mirza, Kamran ............................ 2020

Misdraji, Joseph ....................... 662, 698

Mishra, Sridhar ............................ 1166

Misra, Sanjeev............................... 1802

Mitchell, Duane........................... 1738

Mitchell, Jeannette ..... 665, 666, 2034

Mitchell, Richard N ..................... 315

Mito, Jeffrey ................................. 1321

Mito, Kumiko..................... 1796, 1798

Mitsuhashi, Tomoko..................... 1817

Mittal, Bharat .............................. 1329

Mittal, Khush ................................1116

Mittal, Pardeep ......... 756, 1794, 1800

Mittal, Shachi .................................. 230

Mittal, Vijay .................................... 292

Mittendorf, Elizabeth ........................ 301

Miura, Katsutoshi.......................... 324

Miura, Shiro ............................... 2142

Miyai, Yumi ..................................... 1936

Miyamoto, Hiroshi ...... 870, 887, 941,

Miyauchi, Akira 95

Mizuguchi, Sarah ...................... 610

Mizutani, Yuki.............................. 1684

Mo, Angela Y ................................. 316

Moatamed, Neda A............ 1216, 1225

Moch, Holger .................................. 291

Mochizuki, Kunio ………………...... 610

Modesitt, Susan C ....................... 1241

Modi, Aakash ……………........... 1919

Modi, Lopa......................................59

Modi, Mitul ................................. 2070
Modi, Nisha...

1797

Modiano, Nir.................................. 676

Mody, Dina .......... 369, 370, 380, 440

Mohammad, Amin ...................... 2041

Mohammad, Mohammad M ...... 1417

Mohammad, Nissreen .................. 1213

Mohammad, Ramzi........................ 739

Mohammed, Shamayel ................ 494

Mohan, Sanjay R.............. 1504, 1510

Mohan, Sankar ............................. 1991

Mohan, Shankar ............................ 1752

Mohanty, Abhinita S ...................... 165

Mohanty, Sambit K ..... 35, 986, 1752 ,

1991

Mohanty, Suravi.............................. 648

Mohd Nasir, Nur Diyana B ........... 278

Mohindra, Shalini ....................... 1322

Mohler, James ................................... 875

Mohlman, Jeffrey S...................... 2067

Moignet, Aline ………………...... 1444

Moisini, Ioana ................................ 418

Moiyadi, Aliasgar......................... 1762

Mojica, Wilfrido.............................. 263

Mojica, Wilfrido D.............. 841, 1644

Molberg, Kyle ..................... 423, 2049

Molina, Julian R................ 1907, 2008

Molina, Maria ……………………... 319

Molina, Ricardo ........................... 1533

Molina-Garicano, Javier ............ 1409

Molinari, Francesca.......................... 570

Mollaee, Mehri............................. 1952

Moller, Henrik.................................. 861

Moloo, Zahir ................................. 1547

Momblan, Dulce …………………... 625

Monaco, Sara E .................................356

Moncada, Guadalupe ...... 1002, 1047,

1220

Moncaliano, Maria C .................... 853

Mondello, Alessia .............................. 81

Monga, Varun..................................... 53

Monjazeb, Arta M ............................ 96

Monnin, Christine .................. 507, 508

Monroe, Rob ................................. 1941

Monroe, Robert ...... 1682, 2116, 2156

Montaser, Laleh ........................... 987

Montecalvo, Joseph..................... 1896, 1903,

Montecino, Carolina ................... 1748

Monteiro, Carmela .............................. 1568

Montero, Angel ........................... 1893

Montero-Fernandez, M

Angeles .....

1980

Montez, David................................... 629

Montgomery, Bruce ......... 1061, 1066

Montgomery, Elizabeth A ............ 679 , $680,761,780,781,798,806$, $811,812,825$

Montgomery, Jeffrey S...... 909, 1049, 1068,1069

Montgomery, Kathleen .... 1323, 2082

Montgomery, Stephen P.................... 1

Montiel, Delia P ........................... 1093

Montironi, Carla...... 625, 1348, 1685,

2139

Moo, Tracy-Ann.......... 126, 127, 128

Moon, James ................................ 1907

Mooney, Kelly L .................................. 567

Moore, David A.............................. 1980

Moore, Erika M........................... 1458, 1459

Moore, Margaret .......................... 1876

Moore, Robert F ............................ 527 
Mora, Nichole ................................. 2049

Morales, Gema N ....................... 1338

Morales, Linden .................. 510, 1367

Morales-Oyarvide, Vicente ....... 1109,

Moran, Cesar................................. 2006

Moran, Sebastian........................... 757

Morash, Chris............................... 1048

Moreira, André.............................. 1962

Moreira, Andre L.............. 1592, 1993

Morellas, Vassilios ....................... 1595

Morency, Elizabeth ............... 419, 420

Moreno, Alvaro .............................. 290

Moreno, Carlos S ........ 988, 997, 998

Moreno, Vanessa ................. 982, 989

Moreno Prats, Mariana................ 1639

Morgan, Elizabeth A ........ 1433, 1509

Morgan, Sarah AL......................... 231

Morgan, Terry …................ 1135, 1849

Morgan, Todd M ......................... 1049

Morice, William G ........... 1467, 1512

Morita, Kohei................... 1796, 1798

Morland, Bruce ............................. 1875

Morote, Juan ................................. 890

Morrell, Megan ............................. 1588

Morris, Don................................... 1183

Morrissette, Jennifer ................... 1356

Morrissette, Jennifer D................ 2047

Morrissey, Colm........................... 2113

Morrow, Monica …............... 285, 286

Morse, Nicole..................... 256, 2145

Moscinski, Lynn..... 1517, 1551, 1553

Moshnicova, Anna ...................... 2108

Mosiane, Pulane N ............................ 1640

Moskalev, Evgeny ........................ 2149

Moskaluk, Christopher A ........... 1583,

2138

Mosko, Jeffrey D.......................... 458

Moslehi, Javid ...................................... 315

Mosnier, Jean-François .................. 646

Mosquera, Juan Miguel......... 77, 882,

$1204,1853,1857$

Mosser, Jean................. 946, 947, 948

Mostafa, Mohamed .............. 752, 753

Mosteller, Brian ............................. 122

Motiee, Angelina.............. 2128, 2155

Motoi, Noriko ...................... 98, 1954

Motoi, Toru ............................... 71, 98

Mott, Sarah L ............................... 1781

Motte, Nelly ................................... 473

Motzer, Robert J............................ 874

Moul, Adrienne ........................... 1369

Mounajjed, Taofic ... 754, 1665, 1680,

1782

Mouret-Reynier, Marie-Ange .... 1107

Mousavi, Shima ………………..... 421

Moussa, Sam ................................. 678

Mozos, Anna .............................. 1475

Mrazeck, Karen.............................. 122

Msadabwe-Chikune, Susan C.... 2025

Mubeen, Aysha ....................... 20, 1568

Mudaliar, Vivek.............................. 511

Mudini, Washington..................... 1640

Mueller, Jeffrey.... 170, 338, 354, 415

Mugabe, Marcellin ......................... 232

Mujacic, Ibro................................... 629

Mukherjee, Abir .............................. 3, 5

Mukherjee, Joydeep .................... 1752

Mukhopadhyay, Sanjay.... 1955, 1966

Mullegama, Sureni ....................... 2054

Mullen, Chelsey A....................... 1841
Mullen, John T ................................ 42

Muller, Kristen E........ 233, 234, 1214

Mulligan, Anna Marie......... 155, 280,

2158

Mulligan, Lois................................. 643

Mullins, Keith ............................... 1602

Mully, Thaddeus.............................. 515

Munkarah, Adnan R................... 1092

Munsell, Mark ................................ 1187

Muppa, Prasuna.......... 755, 942, 990,

1490,1956

Muqbil, Irfana .............................. 739

Muraki, Takahashi....................... 1778

Muraki, Takashi ......... 382, 714, 756, $784,837,1794,1799,1800,1804$

Murali, Rajmohan ......368, 1144, 1152

Muralitharan, Sharmini ................ 215

Murata, Yasuhiro ........................... 1816

Murdock, Tricia........................... 1215

Murga-Zamalloa, Carlos ........... 1460,

$\begin{array}{lr} & 1541 \\ \text { Murphy, Kathleen M } & 1231\end{array}$

Murphy, Stephen J ............................ 990

Murray, David L.......................... 2021

Murray, Melissa ... 134, 135, 220, 243

Murray, Sarah S ......................... 1754

Murro, Diana .................................. 1604

Murugan, Paari.......... 437, 991, 2076

Musa, Stephen I ............................. 253

Musallam, Sami G .......................... 1686

Mustafa, Mohamed ........................ 561

Mustafa, Sara …………………...... 402

Musulen, Eva ................................ 757

Mutch, David G ........................... 1131

Mutuiri, Anderson ........................ 1547

Muvunyi, Jean Baptiste................. 232

Muzikansky, Alona .......... 1763, 1993

Muzzafar, Tariq .......................... 1353

Mwaba, Catherine ................................. 2025

Mwala, Kabisa ............................. 2025

Mweemba, Isaac ......................... 2025

Myers, Jeffrey L....... 590, 1050, 2007

Naab, Tammey J..................... 192, 193

Naber, Stephen ............................... 214

Nabeshima, Kazuki .......... 1942, 1980,

2011

Nademanee, Auayporn ................ 1519

Nadji, Mehrdad ........ 194, 1217, 2133

Nael, Ali .................. 1461, 1462, 1891

Nafa, Khedoudja ............................... 79

Nagales Nagamos, Rochelle ......... 422

Nagarajan, Priyadharsini..... 525, 533, $537,1758,2018$

Nagayasu, Takeshi ..................... 1958

Nagendran, Jayan........................... 1914

Nagzarkar, Sudaiv ....................... 1339

Nahas, William C .......................... 959

Naidoo, Jarushka.............................. 2003

Naik, Padmaja ................................. 435

Naina, Harris .......................................... 1378

Naini, Bita V ... 690, 801, 1713, 1786,

2039

Nair, Asha............................. 74, 689

Nair, Navya ..................................1112

Nair, Suresh....................................... 722

Nair, Vidhya ................................. 318

Naiyer, Nada ...................................... 1888

Naizhen, Xu ....................... 982, 989

Najera, Leticia.............................. 1519

Najjar, Saleh N ............................ 992

Najmuddin, Mufaddal ................... 572
Nakagawa, Mayumi ....................1118

Nakai, Tokiko............................. 1632

Nakajima, Naoki ....................... 1957

Nakajima, Tomoyuki.................... 758

Nakamura, Sayuri ........... 1958, 2001

Nakamura, Shigeo....................... 1468

Nakanishi, Kuniaki .................... 1572

Nakasaki, Manando.................... 2140

Nakashima, Masahiro ................. 2142

Nakashima, Megan O.................. 759

Nakazawa, Tadao .......................... 610

Nakhleh, Raouf E............. 2068, 2069

Naklowycz, Al............................... 515

Nalbantoglu, ILKe ........... 726, 1660,

1677,2051

Nalesnik, Michael A................... 1588

Nalisnik, Michael....................... 1760

Nam, Anna ............ 1463, 1464, 1465

Nam, Soo Kyung........................... 716

Namer, Brenda L.......................... 1996

Nandeesh, NB .............................. 648

Nanus, David M ......................... 1857

Naporo, Simon ............................ 1097

Naqvi, Asghar ........... 544, 760, 1580

Narasimhamurthy, Mohan............ 235

Nardi, Valentina .... 1109, 1363, 1931,

1977

Narick, Christina M ......... 339, 1285,

1822,2111

Narula, Navneet ............... 1937, 1938

Nash, Rebecca........... 417, 432, 1778

Nasir, Aqsa .................. 21, 942, 1956

Nason, Katie S ............................. 642

Nasr, Christian ............................ 604

Nasr, Michel.................... 1483, 1519

Nasr, Samih H .................. 1619, 1626

Nasri, Elham ..................... 775, 2045

Nassar, Aziza................................ 290

Nassiri, Mehdi....... 1466, 1523, 1531

Nast, Cynthia............................. 1623

Natkunam, Yasodha ......... 1472, 1832

Natu, Ashvini .............................. 1324

Naum, Bruna .................................... 399

Naumaan, Anam.......................... 1325

Navale, Pooja .................. 1590, 1801

Nava Rodrigues, Daniel ............... 993

Navarro, Alba ............................... 1348

Nayak, Anupma............................. 103

Nayar, Ritu ......... 419, 420, 454, 464,

490,1256

Nazeer, Tipu ...... 944, 992, 994, 1005 , $1015,1391,1392,1654$

Nedjadi, Taoufik.......................... 851

Neel, Benjamin .......................... 1275

Neely, Cameron.... 177, 218, 228, 229

Neff, Jadee ......................... 535, 1467

Neil, Kevin .................................... 750

Neill, Stewart ................... 1737, 1759

Nejati, Reza................................. 1355

Nelis, Mischa ............................... 1583

Nelson, Gregg S .......................... 1184

Nelson, Jonathan ..................... 518

Nelson, Peter ............................... 1066

Nelson, Peter S............................ 1061

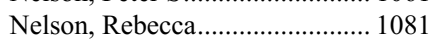

Neppl, Christina .......................... 1959

Nesi, Gabriella ....................... 596, 819

Nestler, Rick................................ 356

Neto, Antonio G

695,840
Netto, George J .......... 941, 951, 961, 1018, 1019, 1056, 1059, 1060,

1127

Neumeister, Veronique................. 340

Newman, Marsali................ 827, 828

Newman, Scott........................... 1890

Newsom, Kimberly J ....... 1675, 1742

Neyaz, Azfar ............................... 1802

$\mathrm{Ng}$, Cedric ........................ 278, 1869

$\mathrm{Ng}$, Cedric Chuan Young ............. 277

Ng, Charlotte K............... 1152, 1854

Ng, Dianna ................................ 1569

$\mathrm{Ng}$, Siok-Bian ............................. 1468

Ng, Tony......... 40, 1189, 1213, 1295,

Ngadiman, Sutini ....................... 2114

Nguyen, Aivi ................................... 319

Nguyen, Doreen .......................... 1850

Nguyen, Kien .............................. 2114

Nguyen, Kim................................ 492

Nguyen, Luong ........................ 1591

Nguyen, Lynh................... 1469, 1553

Nguyen, Michaela ......................... 126

Nguyen, Van T ............................ 2053

Nguyen, $\mathrm{Vu}$.................................. 1613

Ni, Ruoyu................................. 1070

Ni, Shujuan .................................. 822

Ni, Yunbi ....................................... 236

Niblet, Angela .................................. 87

Nichols, Kim ................................. 1890

Nicholson, George ........................ 260

Nicka, Catherine .......................... 1599, 1960

Nickeleit, Volker ........................ 1627

Nickless, David .................... 827, 828

Nickols, Hilary .................................. 57

Nicol, Christopher.......................... 643

Nie, Kui........................................ 1463

Nielsen, GP

1109

Nielsen, G Petur

.. 42, 62

Nielsen, Torsten ................... 40, 1253

Niero, Monia

Niki, Toshiro ............................... 1798

Nikiforov, Yuri ... 425, 582, 600, 1730

Nikiforova, Marina N......... 467, 600, $806,1006,1730,1813$

Nikowitz, Janet ......................... 1519

Niland, Joyce............................... 1519

Nine, Jeffrey S............................ 1587

Ning, Jing..........533, 537, 1193, 1194

Nip, Felicia................................. 2070

Nirschl, Jeffrey................................ 2141

Nishikawa, Toshio......................... 317

Nishino, Ha ................................ 1363

Nishino, Michiya.......................... 358

Nishioka, Hiroshi ......................... 617

Nitta, Hiroaki .............. 180, 212, 213

Niu, Nifang .................................. 629

Niu, Shuang..................................... 423

Nix, Jeffrey.................................. 1041

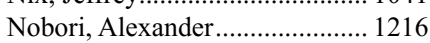

Nodit, Laurentia ........................... 2055

Noë, Michaël

Noel, Jean-Christophe.................. 1128

Nofech-Mozes, Roy ...................... 963

Nofech-Mozes, Sharon ........ 231, 298,

1160,116

Nohle, David G ........................... 1601

Nolan, Sheila............................... 1565

Nolan, Sheila M ......................... 1563

Nolte, David A ............................. 237

Nomani, Laila $\quad 414,424$ 
Nonaka, Daisuke........ 597, 762, 1961 Nonell, Lara ................................ 1875 Nonogaki, Hirofumi........ 1019, 1059, 1060

Noon, Aidan P ............................. 1070

Nora, Virgilita ................................. 1462

Norgan, Andrew .......................... 1570

Normand, Charles ......................... 435

Norquist, Barbara ........................ 1248

Norton, Larry ........................ 285, 286

Nosé, Vania ...................................... 581

Notta, Faiyaz ................................. 1835

Nour, Sherif G....................... 910, 911

Nout, Remi A ................................1113

Nowak, Jan A ................... 2068, 2069

Nowak, Jonathan A ............ 785, 1803

Noyes, Lauren ............................... 410

Nozad, Sahar................ 238, 763, 994

Nozaka, Hiroyuki .......................... 764

Nucci, Marisa R .....1162, 1163, 1222,

$1233,1242,1268$

Oak, Jean...... 1452, 1471, 1472, 1473

Obadofin, Omobolade ................... 528

Obaid, Nourah M ........................... 738

Obeng, Rebecca C............... 995, 1804

Oberley, Matthew......................... 1461

O'Brien, Roisin ................................ 435

O'Broin, Anne Marie ..................... 390

O'Bryan, Brittaney E ...................... 855

O'Callaghan, Daniel ..................... 120

Ochiai, Atsushi ............................... 797

Oda, Yoshinao .... 97, 838, 1942, 2011

Odashiro, Patricia P..................... 1962

O'Donovan, Maria ........................ 1834

Odukoya, Abdullateeph A............... 13

Oduro, Kwadwo....................... 22, 765

Odze, Robert D .... 692, 726, 776, 785

Offermann, Anne........................... 996

Ogan, Kenneth ................................ 1648

Ogata, Sho.................................. 1572

Ogawa, Hiroshi ............................. 1238

Ogawa, Masumi …………………....... 71

Ogilvie, Travis................................ 1183

Ogino, Shuji ................................. 1803

O'Gorman, Maurice RG ............ 1712

Oguntebi, Emmanuel E.................. 767

Ogurtsova, Aleksandra........... 43, 282

Oh, Soo-young ................................. 1181

O'Hare, Kevin................................. 1326

Ohashi, Kenichi..................... 58, 2002

Ohbayashi, Chiho........................ 1632

Ohgami, Robert.... 1365, 1366, 1404, $1419,1420,1437,1471,1472$, $1473,1505,1506$

Ohike, Nobuyuki............................ 756

Ohnoda, Takashi........................... 2142

Ohori, N Paul ........................ 425, 600

Oishi, Naoki ........................................ 610

Ojanguren, Isabel ......................... 1875

Ojo, Olusegun S .............................. 767

Ok, Chi Y ........................ 1488, 1539

Okamura, Keisuke........................ 1817

Okaniwa, Shinji............................ 1794

Okimura, Masato............................ 1249

Okonkwo, Nkechi ......................... 766

Okuda, Masaya .............................. 1936

Okudela, Koji................................. 2002

Okuma, Tomotake............................. 71

Olah, Friday ………………….......... 767

O'Leary, John J ....... 435, 1828, 1860 ,

2132
O'Leary, Matthew ........................... 1588

Olevian, Dane ................................ 768

Olgaard, Ericka ………………..... 2134

Olgac, Semra............................... 1055

Oliva, Esther ...........1094, 1109, 1110, $1113,1125,1186,1244,1245$,

1265,1266

Olivan, Mireia ........................................... 890

Oliveira, Andre.......................... 16, 39

Oliveira, Gabriel LP..... 862, 863, 864

Oliveira, Rafael F......................... 138

Oliver, Bill ........................................... 8

Oliver, Dwight .............................. 1818

Olivier, Magali ............................. 1821

Olofson, Andrea .................. 769, 1599

O'Loughlin, Mark ........................... 120

Olsen, Randall ............................... 369

Olson, Gwyneth ............................. 1930

Olson, Jeffrey J ………………..... 1760

O'Malley, Dennis P......... 1438, 1470,

O'Malley, Margaret....................... 706

Omarzai, Yumna.................................... 14

Ombrello, Amanda ....................... 1358

Omman, Reeba.............................. 1476

Ondič, Ondrej... 849, 983, 1054, 1178

Ondracek, Rochelle Payne ......... 1907

Onega, Tracy ................................. 2032

O'Neil, Maura ................................ 1807

O'Neill, William C.......................... 313

O'Neill, Zachary .................................. 4

Onernek, Ayse Mine....................... 581

Ong, Menchu.................... 2017, 2105

Onisko, Agnieszka ... 141, 1174, 2103

Onodera, Courtney ................ 199, 748

Onozato, Maristela ........................ 1585

Oprea-Ilies, Gabriela....................... 252

Opyrchal, Mateusz ................ 210, 211

Orain, Michèle ...... 1925, 1940, 1962,

1975,1987

Orazi, Attilio .......... 1369, 1452, 1463

Ordi, Jaume ............................. 9, 1573

Ordobazari, Atousa .... 195, 475, 1217

Ordulu, Zehra .................... 1266, 1963

O'Regan, Esther.......................... 1326

O'Regan, Ruth ................................. 208

O'Riain, Ciaran ............................ 1860

Ormenisan Gherasim, Claudia .... 997,

1964, 2143

Ormenisan-Gherasim, Claudia..... 998

Orsi, Nicholas ............................... 528

Ortiz-Martinez, Fernando .... 158, 159

Ortiz, Juan .................................. 1729

Oshilaja, Olaronke ....................... 373

Oshima, Kiyoko ....... 329, 1672, 1769 ,

1770,1810

Oshiro, Hisashi.............................. 1798

Osmani, Lais ....................... 426, 1474

Ostry, Avrum J................................. 10

O'Sullivan, Roderick J......................... 467

Osunkoya, Adeboye .................... 1032

Osunkoya, Adeboye O ............... 417, 432, 894, 910, 911, 915, 927, 988, $995,997,998,1622$

Ota, Hiroyoshi.................... 758, 1102

Otani, Tomoyuki ........................... 1632

Otero, Dennise E.............................. 2144

Otero, Jose...................................... 1611

Otis, Christopher N ..................... 1841

O'Toole, Sandra ........................... 1305
O’Toole, Sharon ...... 435, 1828, 1860,

2132

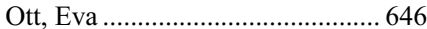

Ottesen, Rebecca......................... 1519

Ou, Joyce.................... 206, 360, 1205

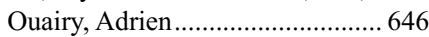

Ouellet, Veronique ......................... 988

Ouseph, Madhu ............................. 1917

Overman, Michael............................. 684

Oweity, Thaira................................. 352

Owen, Scott R ................................... 726

Owens, Christopher L ..................... 451

Ownbey, Robert T .......................... 1095

Owonikoko, Taofeek K .................. 362

Oza, Amit ................1137, 1180, 1185

Oza, Nikita S ............................... 1311

Ozerdem, Ugur..... 239, 240, 241, 269

Ozkaynak, Mehmet...................... 1871

Ozluk, Yasemin ............................... 1633

Ozogul, Ece........................................ 61

Pabedinskas, Dana ......................... 466

Padera, Robert............. 314, 315, 1757

Padgett, Jessica ............................... 566

Padmanabhan, Vijayalakshmi ..... 413, $421,460,462$

Paessler, Michele .......................... 1544

Pagan, Carlos .................... 1928, 1965

Pagano, Nico ............................... 1780

Pai, Reetesh........ 676, 720, 768, 2033

Pai, Rish K .......................... 662, 1656

Pai, Trupti..................................... 1311

Paik, Seung S ......................... 254, 255

Paintal, Ajit ..................... 1256, 1329

Pakuts, Benjamin .......................... 155

Pal, Sumanta ... 994, 994, 1005, 1015,

Palacios, Diana........................... 1574

Palanisamy, Nallasivam ..... 943, 1078

Palapattu, Ganesh S ......... 1049, 1069

Palazzo, Juan P.................... 186, 685

Palisoc, Maryknoll ....................... 1363

Pallavajjalla, Aparna ..................... 1555

Palma Diaz, Miguel F ................. 1651

Palme, Carsten E.......................... 1330

Palmer-Arontsen, Beatrix... 930, 1085

Paludo, Jonas................................ 1396

Pambuccian, Stefan....... 74, 332, 333, $351,427,428,443,444,445$, 936, 957, 968, 1578, 1704, 1826,

Pan, Liuliu.................................. 2156

Pan, Xiaochuan ………………...... 170

Pan, Yongsheng................................... 1609

Panarelli, Nicole C....................... 2016

Panaretakis, Theocharis ... 1354, 1492

Panchal, Hinaben J......................... 854

Pandey, Santosh ................... 35, 1752

Pandita, Ajay ....................... 1752, 1991

Pane Foix, Maria ............................. 849

Paner, Gladell P............................ 876, 877, 925

Pang, Brendan .............................. 1196

Pang, Judy ............... 401, 2007, 2046

Pankajakshan, Chandroth Navin.

Anio, Andrea ....................... 1966

Panse, Gauri ....................................... 72

Pantanowitz, Liron ............................ $1587,1588,1589$

Pantavaidya, Gouri...................... 1289

Pao, Andy .......................................... 734

Paoletti, Costanza

123
Papachristou, Georgios I.... 467, 1813

Papadodima, Stavroula ................ 320

Papadopoulos, Nicholas.............. 1238

Papadopoulos, Nickolas................ 1018

Papaleo, Natalia ......................... 1475

Papaleo, Natalia F .............................. 355

Papanastatiou, Anastasios D ........ 161

Papanikolopoulos, Nikolaos P ... 1595

Papaxoinis, George ............ 762, 1961

Papay, Judit ................................... 1944

Papenhausen, Peter R .................... 1469

Paquette, Cherie ....... 272, 360, 1205 ,

1206,1208

Parakh, Rugvedita ...................... 2071

Parameshwaran, Vishnu ................ 861

Pardeshi, Vishakha ........................ 175

Paré, Guillaume............................. 318

Pareja, Fresia....... 135, 207, 220, 242,

243,1312

Parian, Alyssa.................................. 780

Parimi, Vamsi.... 936, 937, 999, 1476,

1600

Park, Do Joong............................ 716

Park, Do Youn.... 724, 770, 771, 1809

Park, Emily ............ 1941, 2116, 2156

Park, Ha Young .................... 633, 636

Park, Hyunjin ................................. 1792

Park, Jason Y.................... 1559, 1561

Park, Ji-Weon ................................... 448

Park, Kay................ 1144, 1244, 1245

Park, Kyung .... 77, 1848, 1851, 1852 ,

1937,1967

Park, William KC......................... 2135

Park, Won-young.............................. 771

Park, Woong-Yang ....................... 131

Parkash, Vinita ..........545, 1141, 1142,

$1143,1164,1165,2072$

Parker, Kwabna ............................ 1436

Parks, WT...................................... 1133

Parra-Herran, Carlos .......... 231, 298, $1117,1158,1159,1160,1161$, $1210,1218,1233,1247$

Parra, James ..................................... 526

Parrilla Castellar, Edgardo R........ 244

Parry, Susan.................................... 791

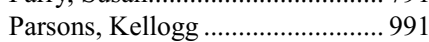

Parvinnejad, Nikoo ........................ 245

Parwani, Anil... 180, 213, 1045, 1192 $1601,1602,1610,1611$

Pasaoglu, Esra ................................ 1784

Pasche, Boris ................................... 2092

Paschke, Ralf.................................... 607

Pascual-Vaca Gumez, Diego ........ 1870

Pasic, Maria........................................ 891

Pastoret, Cedric .............................. 1444

Patel, Ami............................ 246, 2073

Patel, Dominic............................. 1805

Patel, Jay L........................ 1382, 1477

Patel, Kepal N …………………..... 343

Patel, Ketan ........................................ 2063

Patel, Keyur P ........... 537, 749, 1374, $1402,1434,1488,1539,1721$

Patel, Mehul ................................. 1919

Patel, Mihir ..................................... 372

Patel, Natalie .......................... 133, 772

Patel, Nikhil ................................. 1524

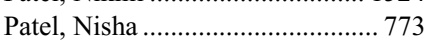

Patel, Nupam.................... 1478, 1489

Patel, Rajiv ……………………..... 519

Patel, Sandip .................................... 2140

Patel, Sanjay........................ 575, 1479 
Patel, Shobhanaben J ..................... 460 Patel, Shweta................376, 476, 926 Patel, Smita ............. 774, 1235, 1283 Patel, Snehalkumar B................... 734 Patel, Tejal........................ 1480, 1889 Patel, Tushar................................. 2083

Patel, Vatsal.......................... 536, 775

Patel, Viren................ 336, 411, 1327

Patell-Socha, Farah .................... 2026

Patey, Martine .............................. 601

Pathak, Ravi R ............................ 1314

Patil, Asawari ......... 1289, 1311, 1324

Patil, Datta..................................... 1622

Patil, Deepa T...... 657, 664, 705, 706,

776,785

Patil, Ninad M.................... 413, 1203

Patil, Pallavi A............ 247, 777, 1968

Patil, Priyanka .............................. 2074

Patil, Suhas.................................. 2114

Pattaras, John G ........................... 995

Pauli, Chantal.................. 1853, 1857

Paulsen, Erika ............................ 1604

Paulsen, John D................. 397, 1969

Paulson, Vera............................... 1746

Pavlisko, Elizabeth N...... 1901, 1902,

1980

Pawlik, Timothy ........................... 1707

Payne-jameau, Yolanda ................ 1328

Payne, Sakeena .......................... 1342

Peace, Janais ................................. 2148

Pearce, Shane M.................. 876, 877

Pearlman, Rachel ................ 694, 746

Pease, Garrison .................. 778, 2075

Peciarolo, Antonella ..................... 1750

Pederson, Holly J .................. 148, 149

Pederzoli, Filippo ............................ 897

Pedron, Serena ……....................... 869

Peevey, Joseph ............................ 1219

Pei, Lin............................ 1922, 2040

Pei, Zhengtong ............................. 598

Peikert, Tobias........ 1956, 1971, 1998

Peiro, Gloria .......................... 158, 159

Peker, Deniz ................................. 1406

Pekmezci, Melike......... 73, 116, 1727

Pelland, Kevin T ......................... 1481

Pellegrinelli, Alessio ............ 594, 595

Pellegrini, Kathryn L..................... 988

Pelletier, Daniel J ................ 553, 1687

Pellise, Maria ................................ 2139

Pelosi, Giuseppe........................... 1970

Pelton, Ashley ............................. 1528

Pemmaraju, Naveen .................... 1355

Pena, Lourdes......... 1002, 1047, 1220

Penault-Llorca, Frederique ......... 248,

779,1107

Pence, Isaac J ................................. 687

Pendse, Avani ................................ 379

Peng, Limin........................... 208, 274

Peng, Xuan............................ 210, 211

Peng, Yu-Ching ................................ 1000

Peng, Yue ...................................... 1482

Pennella, Antonio ......................... 1970

Penny, Will .................................... 1001

Pentecost, Brian T ........................ 1841

Penzias, Gregory ........................... 1603

Pepe, Carmela ............................... 2005

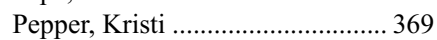

Pepper, Michael ................................. 67

Pereira, Daniela.............................. 249

Perez Montiel, Delia ........... 849, 983, 1002, 1047, 1054, 1220
Pérez-Míes, Belén ......................... 183

Pérez-Montiel, Delia ................... 1101

Perez, Francisco …………............ 359

Periakaruppan, Ramayee............. 1082

Perner, Juliane .............................. 1834

Perner, Sven ........................ 996, 1314

Pernicone, Elizabeth .......................... 9

Perret, Raul .................................. 522

Perrino, Carmen ............... 1003, 1004

Perrone, Anna Myriam................ 1199

Perry, Anamarija .............. 1483, 1519

Perry, Ann E ................................... 532

Perry, Arie ................. 73, 1727, 1753

Perry, Cynthia ………………….... 283

Perry, Kyle D ............ 74, 75, 76, 689

Pesci, Anna..............1110, 1244, 1245

Pestalozzi, Bernhard ..................... 291

Peterson, Jason D ...... 523, 769, 1960,

2129

Peterson, Jess F ............................ 2081

Petersson, Fredrik ...................... 1054

Petros, John A........................ 988, 995

Petrovic, Ivana ................................... 65

Petrowski, Kelly.......................... 1464

Petrozza, Vincenzo............................. 66

Pettaway, Curtis .......................... 1064

Pettus, Jason ................................. 2037

Peyronnet, Benoit......... 946, 947, 948

Peyster, Eliot ................................ 2141

Pezhouh, Maryam ....... 679, 680, 780, $781,798,811,812,825,1688$

Pezzuto, Federica ........................ 1970

Pfarr, Nicole ................................. 1791

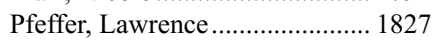

Pfisterer, Jacobus.......................... 1201

Pham, Trinh.................................. 435

Phelan, Sine..................................... 120

Philip, Philip ................................. 739

Philips, Sharon ............................... 1651

Phillips, Jordana ............................. 284

Piao, Jin.......................................... 533

Piazza, Yelena .................... 429, 1806

Picci, Piero .......................... 46, 47, 83

Piccoli, Anthony L ..................... 1589

Pichardo, Janine .......................... 1368

Picken, Maria ................................. 937, 957

Picken, Maria M......... 936, 999, 1600

Piedras, Diana ................... 1028, 1029

Pierce, Kirsten................................. 430

Pietanza, Maria C........................ 1911

Pietenpol, Jennifer............................. 164

Pietzak, Eugene J ......................... 850

Pigeon, Marc-Antoine.................. 1925

Piha-Paul, Sarina A ........................ 147

Pihan, German............................... 1997

Pijuan, Lara .................................. 1955

Pileri, Stefano............................... 1390

Pillai, Raju................ 492, 1518, 1519

Pillai, Vinodh ............................. 1544

Pillappa, Raghavendra ................. 1971

Pilley, Jacob ....................... 1484, 1508

Pina-Oviedo, Sergio .......... 1402, 1485

Pincus, Jennifer............ 166, 288, 289

Pineau, Marion............................. 2125

Pinheiro, Celine M ......................... 959

Pinheiro, Katherine ...................... 1005

Pinho, Daniella............................... 1818

Pinkus, Geraldine ............. 1439, 1455

Pinnaduwage, Dushanthi..... 155, 280,

2158

Pins, Michael R

... 624
Pintille, Melania ............................. 1009

Pinto, Amanda C .......................... 648

Pinto, Andre ...... 90, 973, 1030, 1217,

1221

Piris, Miguel................................. 1390

Pirzada, Amrah.............................. 1218

Pisano, Annalinda ....................... 1723

Pisapia, David J.... 19, 77, 1853, 1857

Piscuoglio, Salvatore............ 207, 243

Pisters, Louis................................ 1064

Pistillo, Maria Pia.......................... 1923

Pitman, Martha B ................. 378, 438

Piton, Nicolas .................... 1972, 1973

Pitt, Susan ..................................... 914

Pittaluga, Stefania ............. 1363, 1390

Pittman, Meredith .............. 782, 1670

Piulats, Josep Maria .................... 1229

Pivovarcikova, Kristyna...... 849, 983 ,

Pizzato, Cristina $\quad 1054$

Pizzi, Marco ………………......... 1486

Pizzo, Salvatore........................... 1701

Plagov, Andrei ................................ 1617

Planas, Ramon............................. 1875

Plant, Barry .................................... 1905

Plant, Pamela............................... 1024

Plata, Adriana ................................... 1401

Plaza, Diego ............................... 1875

Plaza, Jose A.................................... 521

Plesec, Thomas..... 414, 663, 664, 705

Plotkin, Anna.................... 1218, 1250

Plotkin, Scott................................. 1763

Plummer, Regina M ................... 2076

Podoll, Mirna B........... 144, 250, 251,

Podoltsev, Nikolai $\quad 1246$

Pohthipornthawat, Natkrita ......... 1243

Point du Jour, Kimberly ....... 252, 599

Poirier, John T ................................ 1911

Poisson, Laila M ......................... 1760

Poklepovic, Andrew ......................... 16

Pokorny, Morgan.......... 884, 885, 886

Pokrovsky, Anatoly ..................... 1845

Polanco Jacome, Evelyn C......... 2077

Polanco, Evelyn Carolina.............. 717

Polano, Maurizio ................................ 81

Poli, Giada........................................ 596

Polito, Humbert.............................. 1795

Polka, Avery ................................... 1460

Pollack, Aron Z ........................... 1318

Pollack, Ian F ................................ 1730

Polski, Jacek............................... 2127

Polydorides, Alexandros D .......... 729, $730,731,736,737,742,843$, $844,1801,1820$

Pomel, Christophe........................ 1107

Ponce, Jordi.................................. 1229

Ponce, Jose J ................................... 158

Ponder, Teresa ............................... 1168

Ponsky, Lee ..................................... 1603

Pontecorvi, Alfredo ........................ 439

Pool, Christopher …............ 613

Pool, Mark....................................... 703

Popnikolov, Nikolay .................... 1440

Poppiti, Robert ....................... 303, 956

Poropatich, Kate................ 1329, 1974

Port, Jeffrey L..................... 1937, 1938

Porter, Teresa C........................... 1259

Porteus, Cory......................... 815

Post, Steven................................... 1001

Poster, Craig.................................. 1701
Poullot, Elsa ................................... 1444

Poulter, Melinda D ....................... 1209

Pourabdollah, Maryam..... 1372, 1487

Poveda, Julio C .............................. 1369

Powell, Simon .............................. 1854

Powell, Suzanne Z ….................. 1721

Powers, Celeste N .... 433, 1288, 1976

Powles, Carrie …………………..... 435

Pozdnyakova, Olga .......... 1381, 1456, $1509,1542,1543,1546,2060$

Prabhash, Kumar.............................. 634

Pradeep, Yashodhara ................... 1166

Pradhan, Dinesh .... 78, 459, 486, 600,

1006,1174

Prajapati, Shyam ........ 555, 557, 562, $1587,1596,1598$

Prakash, Sonam............................ 1681

Prandi, Davide............................... 1853

Prasad-Hayes, Monica .................1112

Prasad, Anil .......................................6. 678

Prasad, Ashish .................................678

Prasad, Manju L .................... 331, 349

Prasad, Rajeev............................... 1151

Prasad, Shilpa................................ 2028

Prashad, Anubha ........................... 2091

Prathibha, Saranya ........... 1007, 1045

Prayson, Richard A............ 1734, 1744

Prendeville, Susan............. 1008, 1009

Presnell, Sharon ............................ 1686

Preston, John ................. 884, 885, 886

Prezant, David J ........................... 1919

Priamo, Francesco......................... 1801

Price, Matt A ………………….... 1264

Prichard, Jeffrey W ........................ 197

Priemer, David ................. 1010, 1075

Priemer, David S ...................... 23, 24

Prieto, Victor G ... 513, 525, 533, 537, $1290,1355,1758,2018$

Primmer, Michael P..................... 2135

Priore, Salvatore........................... 1011

Priori, Roberta................................. 1723

Prisciandaro, Elena ....................... 1970

Pritchard, Colin ............................. 1066

Pritt, Bobbi S................................ 1570

Prochaska, Michael A..................... 431

Procop, Gary W............................. 2050

Proe, Lori A........................................ 25

Prokhorova, Ina................................ 1064

Pruthi, Deepak K........................... 1638

Przybycin, Christopher ... 1012, 1079,

1085

Puccio, Ignacio............................. 1805

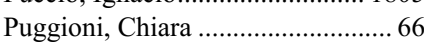

Pulido, Jose .................................. 1716

Pulitzer, Melissa............................. 1368

Pullarkat, Sheeja T ............................. 2054

Pullman, Eli................................... 897

Pullman, James M.............................. 1651

Punzi, Alessandra ......................... 1970

Purushothaman, Sonya................... 783

Purysko, Andrei............................ 1085

Pusceddu, Sara ............................... 595

Pushker, Neelam .......................... 1335

Putra, Juan............. 1689, 1690, 1691

Qasem, Shadi A............................ 2092

Qian, Zhirong ................................ 1803

Qin, Jia ............................. 707, 2078

Qin, Ya-Zhen ................................ 1538

Qing, Xin.................................... 1482

Qiu, Hong........................................ 96

Qiu, Xiaoming............................... 1088 
Qu, Hong

1634

Qu, Zhenhong .................. 1664, 2079

Quade, Bradley J... 1211, 1212, 1222, $1233,1242,1247,1268$

Quddus, M Ruhul................ 206, 247, 272, 360, 939, 1098, 1099, 1205,

1223,1273

Quesada, Andres E...................... 1488

Quezado, Martha M ....................... 626

Qui, Hong..................................... 1383

Quick, Charles.............................. 1001

Quick, Charles M...1118, 1143, 1163,

1169

Quigley, Brian .................... 837, 1795

Quinn, Courtney E ........................ 349

Quinones, Alexander K.................. 300

Quintal, Maisa M .......................... 864

Quintanilla-Martinez, Leticia..... 1507

Quinton, Paul ................................ 727

Quirke, Philip................................ 672

Quitllet, Francesc A....................... 355

Qureshi, Sajid................................. 80

Raab, Stephen .......... 547, 2024, 2091

Raab, Steven ................................ 412

Rabban, Joseph .......1122, 1171, 1176,

1264

Rabban, Joseph T ....... 171, 267, 1141

Rabinovitch, Peter S.............. 661, 817

Raca, Gordana.............................. 1461

Racila, Emilian............................... 1908

Racine Brzostek, Sabrina ............ 1747

Racine, Étienne ............................ 1975

Raciti, Patricia .............................. 1489

Rae, James M ................................. 123

Raess, Philipp W ......................... 1473

Raffeld, Mark .............................. 1390

Raghavendra, Ashwini ................ 1854

Rahaman, Jamal ...........................1112

Rahi, Hamed ................................... 942

Rahimi, Mahdi ............................... 275

Rahimi, Nader ............................... 832

Rahmeh, Tarek ………………..... 2091

Rahmy, Amal.................................. 898

Rai, Harpreet ................................... 316

Rais-Bahrami, Soroush ....... 878, 918,

1041

Raj, Rishi......................... 1906, 1974

Rajasegaran, Vikneswari.... 277, 1869

Rajeshwari, Madhu ..................... 1566

Rajoria, Gaurav ….......................... 860

Rajyalakshmi, Luthra................. 1434

Rakha, Emad A.............................. 260

Rakislova, Natalia .............. 625, 2139

Raleigh, David ........................... 1727

Ralston, Jonathan S ........................ 273

Ram, Rosalyn ............................... 1818

Ramadwar, Mukta ....................... 1797

Ramakrishna, Rohan ....................... 77

Ramalingam, Preetha .......1136, 1140,

1150

Raman, Jay.................................. 1074

Ramani, Nisha S............................ 529

Rambau, Peter F.......................... 1183

Ramella, Aman............................... 373

Ramirez-Santrich, Cecilia ......... 1466,

1523

Ramirez-Villar, Gema L.............. 1870

Ramirez, Daniel C........................... 79

Ramirez, Yuly................................. 1013

Ramis-Zaldivar, Joan Enric........ 1409

Ramjeesingh, Ravi.

643
Ramkissoon, Shakti.......... 1880, 1924

Ramkissoon, Shakti H......... 196, 238, 496, 790, 994, 1005, 1015, 1138, $1297,1331,1654,1836,1855$,

1920

Ramon y Cajal, Santiago... 890, 1748,

$\begin{array}{rr} & 1983 \\ \text { Ramos-Oliver, Irma } & 1748\end{array}$

Ramos, Benjamin D ........................... 25

Ramos, Juan Carlos..................... 1431

Ramos, Robert............................. 1930

Ramsey-Goldman, Rosalind ...... 1526

Rane, Neha .................................. 1919

Rane, Swapnil .......... 634, 1289, 1797

Rangan, Aruna.................. 1490, 1491

Ranganathan, Sarangarajan............ 78

Rangel, Laureano J......................... 990

Ranheim, Erik ................................ 556

Ranson, Marie........ 1305, 1330, 1333

Rao, Arundhati .... 102, 188, 510, 609, $1168,1367,2041,2064$

Rao, Deepthi ...... 668, 732, 733, 1807

Rao, Jianyu.................................... 334

Rao, Koneti ................................. 1535

Rao, Lokinedi............................... 1052

Rao, Priya........ 922, 962, 1022, 1026

Rao, Rema A .... 364, 576, 1853, 1857

Rao, Sambasiva.....................635, 674

Rao, Uma NM................................. 78

Rao, Veena N................................ 252

Raparia, Kirtee ....... 1906, 1974, 1981

Raphael, Solomon ......................... 253

Rashid, Asif............. 533, 1777, 1785

Rassaei, Negar............................. 2058

Rassidakis, George........... 1354, 1492

Rastgoo, Nasrin............................ 1537

Rastogi, Prerna ................................ 1645

Rathkopf, Dana E......................... 935

Ravaud, Alain............... 946, 947, 948

Ravetti, Jean Louis...................... 1923

Ravindran, Aishwarya..... 1641, 1642,

$\begin{array}{ll} & 1643 \\ \text { Ravinsky, Esther } & 2091\end{array}$

Rawish, Kojo.................................. 1224

Raychaudhuri, Pradip................. 2148

Razavi, Pedram ............................... 165

Rea, Bryan................................... 1493

Reading, Noel S .......................... 1520

Rech, Karen L ................... 1491, 1554

Rechsteiner, Markus....................... 291

Reddy, Avinash K............................ 854

Reddy, Opal L .................... 334, 1225

Reddy, Vijaya B ............... 1235, 1308

Reder, Nicholas ............................ 1673

Reder, Nicholas P.... 219, 1014, 1226,

2080, 2137

Redig, Amanda J .......................... 1926

Redman, Mary W......................... 1907

Redmond, David ........................... 1463

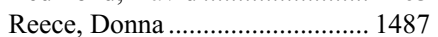

Reeve, Jeff.................................... 1614

Reeves, Ward....... 338, 354, 415, 416,

Rehder, Catherine $\quad 483$

Rehman, Abdul ..................... 254, 255

Rehman, Aseeb ................. 1005, 1015

Rehrauer, William ......... 29, 377, 591,

619,2117

Reichard, Kaaren K.......... 1375, 1494
Reid, Michelle D......... 382, 417, 432, $671,714,756,784,787,837$, 1773, 1778, 1786, 1794, 1795, $1799,1800,1804,1808$

Reiff, Daniel................................. 565

Reinig, Erica F ....... 1375, 1491, 1494

Reis-Filho, Jorge ......... 161, 207, 220, $242,1152,1312,1854$

Reis-Filho, Jorge S....................... 243

Reis, Gerald................................... 1727

Reis, Sabrina T .............................. 959

Reisenbichler, Emily ........... 250, 548,

2082

Reith, John D ............................... 536

Rekhi, Bharat .................................... 80

Rekhtman, Natasha ........... 1903, 1910, $1911,1953,2003$

Remotti, Helen E....... 675, 783, 1678,

1679,1693

Ren, Hongzheng............................ 74

Ren, Kevin Yi Mi ........ 256, 257, 643,

2145

Ren, Qinghu ............................... 1016

Ren, Rongqin ........... 433, 1288, 1976

Ren, Shuyue ................................... 434

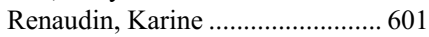

Renda, Valentina .............................. 70

Rendi, Mara H.................. 1248, 2032

Renne, Giuseppe ............................ 879

Renne, Salvatore ……....................... 879

Rennspiess, Dorit ............................ 509

Renouf, Daniel J................................ 1787

Renshaw, Andrew ......................... 474

Resetova, Erika …………………..... 1846

Reshetnyak, Yana K ................... 2108

Resnick, Murray B...... 295, 700, 747 ,

831,977

Resteghini, Nancy ......................... 284

Reuss, David E................................... 81

Reuter, Victor E........... 850, 874, 916, $935,961,985,1037,1038,1053$, $1055,1058,2133$

Revelo, Monica P......................... 1637

Revetta, Frank ………...................... 744

Rex, Douglas K............................... 726

Reynolds, Jordan P...................... 473, 414, 424

Reynolds, Stephen......................... 435

Rezaee, Neda........................... 402, 403

Rezaei, M Katayoon..................... 1019

Rezk, Sherif................................. 1462

Rhines, Laurence D..................... 93, 94

Riad, Sara ..................................... 963

Ribalta, Teresa................................ 1893

Ribeiro, Agnes.............................. 1128

Riben, Michael W .............................. 366

Ricceri, Valeria............................. 1723

Ricci, Claudio .............................. 1780

Ricci, Costantino............................ 1780

Rice, Kevin R................................. 871

Richard, Hope …………………......... 16

Richard, Scott D.......................... 1095

Richards, Stephanie................ 258, 259

Richardson, Aida ........................... 1495

Richon, Catherine .......................... 473

Ricketts, Christopher...................... 982

Rickman, David S ........................... 77

Ricks-Santi, Luisel J ......................... 192

Ridaura-Sanz, Cecilia ...................... 26

Riddell, Robert H......... 672, 673, 692

Riedel, David .................................. 258

Rieder, Florian .............................. 829
Righi, Alberto..................... 46, 47, 83

Righi, Simona ............................. 1486

Rijk, Anke F ................................ 2005

Riley, Meghan ................................ 1496

Rimm, David L .............................. 340

Rimsza, Lisa..................................... 1514

Rinehart, Elizabeth M ................... 785

Ring, Kari.......................... 1155, 1241

Rios, Alyicia................................. 2026

Rioux-Leclercq, Nathalie.... 884, 885, $886,946,947,948,1065$

Rishi, Arvind ................................... 799

Ristow, Kay M ................................. 1652

Ritchie, Allison ........................... 2036

Ritterhouse, Lauren.......... 1809, 1977

Rittscher, Jens ................................ 260

Rivera-Colon, Glorimar .............. 1227

Rivera, Michael.......................... 16062

Rizo, Annnaliza............................... 232

Rizvi, Naiyer ..................... 1928, 1965

Rizzo, Stefania ..................... 321, 322

Ro, Jae.......................... 36, 224, 328

Roa, Juan C .................................. 1804

Roa, Juan Carlos ..... 784, 1774, 1778

1795,1808

Robert, Marie ....................................... 652

Roberto, Gamez G ......................... 351

Roberts, Amity L......................... 1733

Roberts, Daniel E......................... 1692

Roberts, Jordan A............................ 786

Robertson, Scott..................... 705, 706

Robertson, Susan J ...................... 1048

Robin-Radosevic, Nina ................ 248

Robinson, Brian .......... 715, 787, 882, $978,1023,1228,1853,1857$,

2073, 2089

Robinson, Cemre............................... 761

Robinson, Daniel............................. 123

Robinson, Robert ..... 552, 1301, 1315

Rocha, Ruana M........... 862, 863, 864

Rock, Colleen................................ 518

Roden, Anja C....... 1929, 1971, 1986, 1998, 2004, 2008, 2013, 2014,

2084

Rodriguez Canales, Jaime............ 125, 1193,1194

Rodriguez Pena, Maria Del Carmen............. 1018, 1019, 1059, 1060,1127

Rodriguez-Alvarez,

Francisco J ............................ 1630

Rodriguez-Canales, Jaime... 301, 952, 2153

Rodríguez-Gómez, Adriana ...... 1028, 1029

Rodriguez-Justo, Manuel ............ 1805 Rodriguez-Urrego, Paula A......... 1574 Rodriguez-Zarco, Enrique............ 1030 Rodríguez, Eva.............................. 1893 Rodriguez, Fausto ..... 44, 1215, 1753, 1761

Rodriguez, Ingrid M........ 1017, 1027, 1028,1029

Rodriguez, Leonardo...................... 359

Rodriguez, Olga ............................... 1874

Rodriguez, Pablo............................... 511

Rodriguez, Sonia .............................. 290

Rodvold, Jeffrey J ........................... 1051

Roe, Catherine J ............................ 1575

Rogers, Thomas ............................ 1648

Roggin, Kevin ................................ 338 
Roggli, Victor L ………………..... 1901

Rogman, Alberic ........................... 855

Roig, Ignasi .................................. 223

Rojas, Claudia .............................. 1783

Rojo, Federico................................ 183

Roland, Joseph T............................. 548

Roland, Lania ................................ 250

Roma, Andres A....... 172, 174, 1093, $1141,1142,1164,1165$

Roman-Holba, Samara................ 1406

Roman, Amanda........................... 1894

Roman, Benjamin ................. 581, 615

Romero, Eduardo ......................... 1607

Romero, Maria E............................ 323

Rominger, Rachel E .................... 1978

Ronan, Nicola ............................... 1905

Roncaioli, Justin L ......................... 467

Roncal, O ...................................... 1306

Roncella, Silvio.......................... 1923

Ronen, Shira............. 845, 1020, 2081

Rong, Juan........................ 1561, 1749

Ronnett, Brigitte M......... 1215, 1231

Ronquillo, Nemencio ..................... 788

Rook, Alain ................................. 1493

Rooper, Lisa M ........ 436, 1057, 1979

Root, Matthew..................... 437, 2085

Roper, Edward ............................... 1330

Roquiz, Woodlyne........................ 1497

Rosa, Gabriela................................. 530

Rosa, Marilin............................... 2038

Rosati, Rachele .......................... 1853

Rose, Gary............................. 258, 259

Rose, Laurel ................................. 1021

Rose, Peter .................................. 1100

Rose, Sarah .................................. 1191

Rosen, Devin............................... 1624

Rosen, Lauren E............... 1980, 1981

Rosen, Milan .................... 1624, 1649

Rosen, Seymour ............. 9, 1624, 1649

Rosenbaum, Jason N.... 602, 605, 619

Rosenbaum, Matthew W..... 378, 438,

789

Rosenberg, Andrew E ... 59, 90, 1221, 2133

Rosenberg, Jonathan E...... 850, 1055, 1058

Rosenblatt, Paula.................. 258, 259

Rosenblum, Marc.................. 55, 1753

Rosendahl, Isabel ........................ 1559

Rosenthal, Barbara ........................ 402

Rosenwald, Andreas.................... 1380

Roshal, Mikhail.......................... 1368

Rosner, Inger L... 871, 872, 912, 1076

Ross, Aidan .................................... 260

Ross, Ashley E ........... 859, 860, 1056

Ross, Dara S.................................. 165

Ross, Jeffrey........ 132, 196, 238, 496, 763, 790, 944, 992, 994, 1005, 1015, 1138, 1297, 1331, 1391, 1392, 1654, 1836, 1855, 1880,

1920, 1924

Ross, Merrick I................................ 533

Rossi, Esther ............. 439, 490, 1277

Rossi, Esther Diana ............... 336, 411

Rossi, Michael.................................. 995

Rossi, Roberta ................................ 879

Rossi, Sabrina ...................... 81, 1750

Rosty, Christophe........ 728, 791, 792,

793

Roth, Christine G ..................... 2100

Rothberg, Paul G...... 773, 1385, 2120
Rotimi, Olorunda …........................ 528

Rotondo, Fabio............................ 1024

Rotterova, Pavla......... 849, 983, 1054

Rouhi, Omid..................... 1759, 2146

Roulston, Diane............................. 1050

Roupret, Morgan ........................... 1070

Routbort, Mark J ....... 537, 749, 1022, $1416,1429,1434,1488,1539$, $1721,1922,2040$

Rouzbahman, Marjan........1109, 1180

Rovira, Jordi................................ 1229

Rowan, Daniel................... 1672, 1810

Rowe, JJ ............................................ 122

Roxanis, Ioannis............................ 260

Roy-Chowdhuri, Sinchita .... 455, 456

Roy, Madhuchhanda ............ 602, 605

Roy, Paromita................................. 794

Roy, Somak .................................... 1006, 1730

Royal, Richard E............................. 533

Roychoudhury, Sudarshana......... 168,

Ruan, Guo-Rui $\quad \begin{array}{r}563 \\ 1538\end{array}$

Ruano, Ana................................... 1332

Rubenstein, Michael ..................... 853

Rubin, Brian................................. 1873

Rubin, Brian P........ 31, 74, 242, 500, $519,534,1312,1583$

Rubin, Mark A... 77, 882, 1204, 1848, $1852,1853,1857$

Rubino, Anthony .... 1606, 1678, 1693

Rubinson, Douglas A ................. 1803

Rubinstein, Paul ........................... 1464

Rubio, Belen L ................................ 355

Rubio, Carlos A............................... 692

Rubio, Isabel T..................... 222, 223

Ruby, Kristen N ................... 531, 532

Rudan, John........................ 256, 2145

Rudin, Charles M........................ 1911

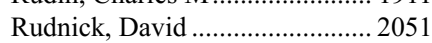

Rudomina, Dorota......................... 404

Ruff, Heather.................................. 440

Rugge, Massimo .............. 1486, 1685

Ruggieri, Pietro ............................... 47

Rugwizangoga, Belson ................ 232

Ruiz-Cordero, Roberto................ 1022

Ruiz, Jeanette .................................. 261

Ruiz, Nuria .................................. 1229

Ruminy, Philippe ............................ 1973

Rupcich, Christine.............. 448, 1604

Rusch, Valerie ............................. 2003

Rush, Natalia..................... 795, 1694

Rushton, Kristen ............................ 518

Russell-Goldman, Eleanor ............ 441

Russell, Donna .............................. 2095

Russell, Maria C............................... 787

Russo, Joshua ............................... 873

Ruterbusch, Julie J ....... 113, 139, 175

Rutgers, Joanne K ...................... 1856

Ruttle, Carmel .............................. 1860

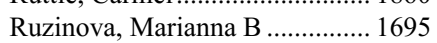

Ryan, Shawnia .................................. 49

Rychly, Boris................................ 1207

Ryder, Jonathan .............................. 565

Ryu, Jay H.................................... 1946

Saad, Ali G......... 75, 412, 847, 1361, $1393,1412,1480,1718,1882$,

1889,2024

Saad, Fred .......................... 988, 2152

Saad, Heba .................................... 1436

Saavedra-Martinez, Jenny ........... 1574

Saavedra, Olivia ............................. 366
Sábado, Constantino .................... 1409

Sabari, Joshua K........................... 1911

Sabattini, Elena ……………….... 1486

Sabourin, Jean-Christophe ......... 1972,

1973

Sabra, Mona ... 616

Sachak, Taha …................... 796, 1696

Sacher, Adrian ................... 1928, 1965

Sacks, Barry A................................. 358

Sacks, Wendy ................................... 611

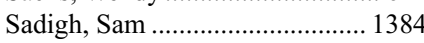

Sadimin, Evita............................... 1605

Sadow, Peter M ............................... 581

Sadowinski-Pine, Stanislaw ........ 1868

Sae-Ow, Wichit ............................. 1903

Saeb Lima, Marcela ........................ 500

Saeed-Vafa, Daryoush................... 603

Saeed, Faisal ........... 904, 1023, 1697

Saeed, Omer........................... 82, 442

Saei Hamedani, Farid......... 262, 1179

Saez, Carmen ................................ 1870

Safdar, Nida ................................. 2082

Saglam, Ozlen ............................ 1230

Saharti, Samah ..... 420, 443, 444, 445

Sahasrabuddhe, Vikrant ............. 1191

Sahin, Aysegul A ......... 125, 147, 208,

Sahoo, Debashis............................ 960

Sahoo, Sunati ................................. 265

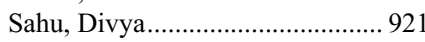

Said, Samar .................................... 1680

Sailer, Verena ..................... 1853, 1857

Saito, Michihiro ............................ 1796

Saito, Norio …………....................... 797

Saito, Ryoko................................. 1982

Sajjan, Sujata...................... 168, 2077

Sakhdari, Ali ..................... 1498, 1811

Sakic, Antonija .............................. 321

Sakr, Hany ................. 604, 1322, 1499

Sakr, Lama ................................... 2005

Sakr, Sharif...................................... 1092

Saksena, Annapurna......... 1500, 1549

Sakuyama, Naoki ........................... 797

Sala, Margarita ............................... 1875

Salama, Mohamed............ 1382, 1386 , 1520

Salamat, M Shahriar............... 29, 602

Salamat, MS ......................................... 619

Salami, Simpa S ............................. 1069

Salaverria, Itziar........................... 1409

Saleeb, Rola ............... 891, 963, 1024

Salem, Alireza .................. 1403, 1501

Salem, Fadi ...................... 1628, 1631, 1653

Salem, Ronald .............................. 1776

Salem, Samira AM .......................... 945

Salfi, Nunzio CM ........................ 1199

Salib, Christian................. 1025, 2147

Salibay, Christine J............. 2083, 2148

Salimian, Kevan .............................. 798

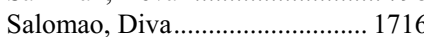

Salona, Beklaswar............ 1752, 1991

Salto-Tellez, Manuel

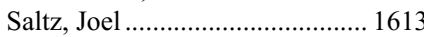

Salvatore, Steven.................... 19, 669

Salvi, Sandra .................................. 1923

Samal, Aurobinda.................. 35, 1991

Samankan, Shabnam ........................ 263

Samant, Sandeep .......................... 1329

Samaras, Dimitris ......................... 1613

Samaratunga, Hemamali ..... 884, 885,

$886,895,896$
Samdani, Rashmi ............... 533, 1026

Samimi, Sara .................................. 1493

Samolczyk, Julia ................... 454, 490

Samra, Hasan ............................... 1087

Samuel, Julian ..................................562

Samuelson, Megan ............... 552, 1781

Sanati, Souzan............................... 1307

Sanchez-Cid, Lourdes .................... 154

Sanchez, Beatriz........................... 1256

Sanchez, Diego F ........... 1017, 1027,

1028,1029

Sanchez, Douglas ................ 890, 1983

Sanchez, Jessica ............................ 1525

Sanchez, Sandra .............................. 1431

Sanchez, Teresa ............................ 1349

Sandell, Rosalind ............................. 264

Sanders, Devin K ......................... 1858

Sanders, Mary Ann....... 119, 191, 250,

Sanders, Melinda $\quad 287$

Sanders, ML ................................ 1645

Sanders, Ronald D ........................ 893

Sanders, Ronda ................ 1030, 1328

Sandhu, Jasmin ............................ 1704

Sandison, Ann .......................... 1282,1292

Sanfrancesco, Joseph ........ 561, 1031,

1032,1033

Sangoi, Ankur .............................. 1012

Sanguino Ramirez, Angela.......... 1034

Sanmartin, Elena .................... 158, 159

Sansano, Irene ................................ 1983

Santacana, Maria........................... 1229

Santagata, Sandro........................ 1726

Santella, Regina M....................... 1907

Santi, Raffaella..................................596

Santiago, Rex Michael ................. 1984

Santiago, Teresa ............................. 1890

Santiago, Victor.............................. 1502

Santin, Alessandro D................... 1138

Santini, Donatella DS....... 1199, 1780

Santos-Zabala, Maria

Laureana........

Santos, Gilda DC

1911

Sanyal, Soma.

4.466

Sanz, Javier

Saornil, Maria A.......................... 1767, 1768

Saqi, Anjali...................... 1928, 1965

Sar, Aylin......................... 1035, 1036

Saravanan, Arthy ……………..... 2035

Sardiña, Luis A .............................. 534

Sargent, Rachel ................................ 1544

Sarin, Kavita …………………....... 518

Sarmiento, Juan M .... 756, 784, 1794 , $1799,1800,1804,1808$

Sarrias, Maria R ……………........ 1875

Sarungbam, Judy.............. 1037, 1038

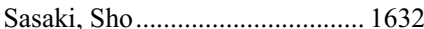

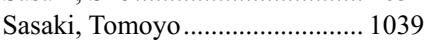

Sasano, Hironobu ........................... 1982

Saskova, Bohuslava …………......... 1054

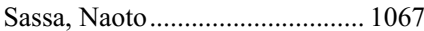

Sata, Naohiro..................... 1796, 1798

Satgunaseelan, Laveniya.............. 1333

Sato, Shun ...................................... 1040

Sato, Shuntaro ............................... 1909

Satturwar, Swati ............................. 564

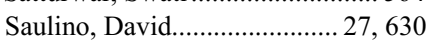

Sauter, Jennifer L .............. 1986, 2084

Savage, Erica................................. 705

Savage, Johanna ........................... 1231

Savant, Deepika .................... 799, 800 
Savio, Antonella .............................. 570 Savona, Michael R........... 1504, 1510 Sawamura, Tatsuya ..................... 1383 Saxena, Romil... 658, 726, 795, 1668,

Sayed, Shahin............................ 1547

Sayeed, Sadia ............................... 1232

Saylor, Benjamin......................... 1041

Sazanova, Olga …....................... 1940

Sazonova, Olga …....................... 1987

Sbaraglia, Marta.................. 47, 81, 83

Sboner, Andrea............ 77, 882, 1204,

1853,1857

Scalia, Carla R ............................ 2136

Scambia, Giovanni ....................... 1277

Scandura, Glenda ........................ 1042

Scapa, Jason V ........... 690, 801, 1988

Scardino, Peter ................................. 861

Scattone, Anna ............................ 1970

Scavuzzo, Anna................ 1002, 1047

Scelo, Ghislaine .................. 846, 1821

Schaberg, Kurt .................. 1698, 1699

Schaefer, Inga-Marie............ 84, 1233

Schaefer, Rachel................... 873, 987

Schaeffer, David.......... 382, 814, 1787

Schaeffer, Edward M ......... 860, 1056

Schally, Andrew V ........................ 194

Schandl, Cynthia A........................ 273

Schechter, Shula........................... 1812

Scheitler, Kristen M ......................... 482

Scher, Howard I .................... 916, 935

Scherr, Douglas S........................ 978

Schiano, Thomas D......... 1689, 1690,

1691

Schieda, Nicola ............................. 1048

Schiffman, Marc H...................... 1857

Schildhaus, Hans-Ulrich ............. 1941

Schimmer, Aaron ............. 1372, 1432

Schittenhelm, Jens.......................... 544

Schlumberger, Martin .................... 473

Schmid, Ralph A .......................... 1959

Schmidt, RA........................................... 645

Schmidt, Robert L.... 958, 2053, 2067

Schmitt, Alessandra...... 372, 479, 599

Schmitt, Fernando ........................ 1312

Schmolze, Daniel ............................. 446

Schneider, Jowaly ............................ 12

Schneider, Sallie S ........................ 1841

Schneider, Sarah............................. 530

Schniederjan, Matthew .... 1737, 1759

Schnitt, Stuart J ................... 121, 284

Schoedel, Karen E................... 78, 425

Schoenfield, Lynn ......................... 1611

Schofield, Kevin..................... 330, 331

Scholar, Paige..................................... 565

Schoolmeester, J Kenneth ............ 1232

Schott, Anne F.............................. 123

Schouweiler, Katie ....................... 1503

Schrage, Matthew ....................... 1700

Schrock, Alexa .... 238, 496, 790, 994, 1005, 1015, 1297, 1331, 1654, $1836,1855,1920,1924$

Schroedter, Ingo ............................ 1483

Schrum, Adam............................... 1467

Schulte, Klaus-Martin .................... 578

Schulten, Hans-Juergen................ 1717

Schultz, Daniel ................................. 410

Schultz, Nicholas ............................ 897

Schultz, Nikolaus ........................... 916

Schumacher, Jonathan...... 1457, 1477

Schuster, David M.......................... 998
Schuuring, Ed................................ 2115

Schwab, Richard ......................... 1865

Schwalbe, Michael............... 602, 605

Schwartz, Lauren E... 560, 857, 1011,

2097

Schwartz, Mary R ....... 224, 328, 370,

Schweizer, Michael..................... 1066

Schwetye, Katherine E..................... 30

Schwock, Joerg ........................... 1180

Sciallis, Andrew .................. 401, 1240

Sciarrone, Salvatore .................... 1685

Scognamiglio, Theresa............... 1376, 134,

2073, 2089

Scott, John P.................... 1929, 2013

Scott, Rona .................................... 1859

Scotti, Mauro................................... 594

Sebastiano, Christopher ................. 447

Sebestyen, Anna ............................. 1944

Sedillo, Javier............................... 2083

Sedivcova, Monika ....................... 984

Seegmiller, Adam.............. 1504, 1510

Seethala, Raja R .......... 425, 582, 600,

$1294,1300,1318$

Segal, Jeremy ........................ 628, 629

Segal, Joshua.................... 1505, 1506

Segal, Neil....................................... 732

Segura, Sheila ............................. 1234

Seheult, Jansen ................................. 459

Seidman, Michael A................ 10, 316

Seipel, Amanda H ......................... 895

Seisen, Thomas ............................... 1070

Seitz, Gerhard ............................. 2149

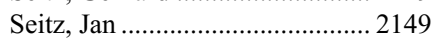

Sekar, Rishi R ............................. 1622

Sekhar, Aarti................................... 756

Sekhon, Harman S .............. 391, 1904

Selenica, Marina ........................... 1597

Selenica, Pier.................................... 207

Selvaggi, Suzanne........ 346, 377, 556

Selvaraju, Suresh B..................... 2144

Sen, Chandra ..................................... 48

Sen, Seema..................................... 1335

Senapedis, William ........................ 739

Senarathne, Wijendra .................. 1989

SenGupta, Dhruba ................................ 1558

Sengupta, Sandip........................... 257

Senz, Anne ..................................... 1401

Seow, Albert..................................... 855

Sepulveda, Antonia ............... 675, 840

Sepulveda, Jorge ..................... 675, 840

Serdy, Kate .................. 140, 209, 1103

Serinsoz Linke, Ebru.................... 1507

Serio, Gabriella ............................ 1970

Serra, Stefano...... 638, 639, 712, 802,

$\begin{array}{rr} & 1789 \\ \text { Serrano, Jonathan } & 306\end{array}$

Sessa, Fausto .................................. 570, 819, 1782

Sesterhenn, Isabell ...... 871, 872, 912,

1076

Seth, Arun ..................231, 1158, 1159

Sethi, Sanjeev......... 1619, 1641, 1642,

$\begin{array}{rr} & 1643 \\ \text { Sethi, Shenon } \quad 448,774,1235\end{array}$

Setia, Namrata............ 338, 354, 415, 628, $629,662,698,718,719,1809$

Seto, Matthew ………………........ 802

Setoodeh, Sasan ....... 265, 1484, 1508

Settembre, Elizabeth .................... 1523

Setty, Suman ............................... 2150

Sexton, Donal................................ 1614
Sfanos, Karen S............................ 2113

Sfreddo, Hannah J......................... 736

Shaar, Mohanad ................ 1043, 1701

Shabaik, Ahmed ............................ 1080

Shabana, Marwa E ........................... 99

Shabihkhani, Maryam ........... 266, 781

Shackelford, Rodney......... 2105, 2154

Shaddy, Sophia M .......................... 803

Shafizadeh, Nafis................. 826, 1705

Shah, Alpa B ………………….... 1044

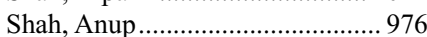

Shah, Jatin .......................................... 65

Shah, Kabeer ...................................... 535

Shah, Mit D .................................. 1045

Shah, Rajah ....................................... 893

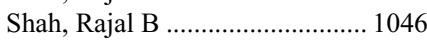

Shah, Ronak H ........................... 1861

Shah, Sejal........................ 1680, 2094

Shah, Varsha I .............................. 1253

Shahi, Maryam............ 449, 450, 606 ,

1236,2085

Shahmarvand, Nahid.................. 1471

Shahsafaei, Ali .............................. 1528

Shaib, Walid ................................. 787

Shaikhibrahim, Zaki....................... 996

Shamash, Jonathan ...................... 1042

Shamir, Eliah................................ 267

Shan, Ling ....................................1111

Shan, Mu ..................................... 2021

Shanbhag, Namita .......................... 1996

Shang, Guoguo............................. 1990

Shanmugam, Vignesh.................. 1509

Shanmugham, Pramesh................. 634

Shao, Haipeng .................... 1517, 1551

Shao, Lina .... 1050, 1363, 1376, 1878

Shapiro, Linda G......................... 2032

Shareef, Mohamed M................... 1751

Sharkey Ochoa, Imogen................ 435

Sharma, Aarti .................................. 2051

Sharma, Anjana ............................ 1335

Sharma, Anupama ........................ 1980

Sharma, Anurag...................... 35, 1752

Sharma, Gaurav............................ 2043

Sharma, Meenal ......... 804, 805, 1702

Sharma, Mehar C ........................ 1566

Sharma, Nitya V ................................. 988

Sharma, Rajni.... 124, 282, 297, 1019, $1059,1060,1127,1979$

Sharma, Rohit ................................. 452

Sharma, Sachein................ 1530, 1536

Sharma, Shivani ............ 35, 986, 1752,

Sharp, Linda ................................. 435

Sharzehi, Setareh......................... 2058

Shaver, Aaron C ..... 1388, 1504, 1510

Shaw, Gene R............................... 1479

Shaw, Kenna ................................ 1824

Shaw, Kristin..................................... 873

Shaw, McKenzie .......................... 1763

Shaw, Patricia..........1126, 1137, 1185 ,

1251,1275

Shaw, Reid ................................... 1853

Sheaff, Michael ................................ 1980

Sheedy, Frederick........................ 1828

Sheehan, Christine E... 132, 238, 763, 944, 992, 994, 1005, 1391, 1392, $1881,1919,2162$

Sheehan, Margaret ........................ 120 Shehatou, Teriza ................................... 1

Sheibani, Khalil..................................... 1930

Sheils, Orla

1828,1860
Shelekhova, Ksenya V ................... 521

Shelton, Jeremy .................................. 28

Shen, Hong......................... 1703, 1711

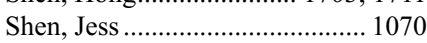

Shen, Li ……………………........ 1846

Shen, Ronglai ........................ 882, 1152

Shen, Rulong................................ 1610

Shen, Tiansheng ............ 169, 268, 296

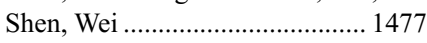

Shen, Yulei ..................................... 1511

Sheng, Dong.................................. 1862

Sheng, Shijie ................................... 740

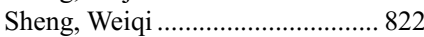

Shepherd, Neil................................. 692

Sheppard, Mary N................ 326, 327

Sherbeck, John P........................ 2086

Sheridan, Todd B........................... 451

Sherpa, Palzum ……...................... 1237

Shervinrad, Mohammadreza ......... 269

Shestakova, Anna ......................... 1462

Shetty, Omshree ................................... 1762

Shetty, Prakash ............................. 1762

Shi, Chanjuan ................ 686, 744, 786

Shi, Jianhui........................ 1682, 1711

Shi, Jiaqi..................... 382, 726, 1812

Shi, Min............................ 1467, 1512

Shi, Mingxia................................. 1859

Shi, Qian ……………………......... 722

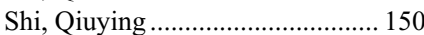

Shi, Wenjing................................... 1317

Shia, Jinru ........... 668, 732, 733, 916,

1861

Shibemba, Aaron L …………….. 2025

Shiekhmohammed,

Mohammed .

Shields, Anthony ........................ 994

Shih, Andrew..................................... 1195

Shih, Angela ........... 1513, 1992, 1993

Shih, Ie-Ming ................... 1238, 1271

Shih, Natalie................................. 1603

Shillingford, Nick ....................... 1891

Shilo, Konstantin............................ 803

Shimizu, Akira .............................. 1943

Shimizu, David M........................ 1259

Shimizu, Ken................................. 1572

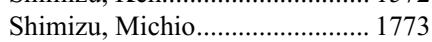

Shin, Eugene T................................ 270

Shin, Junyoung................................ 1239

Shin, Kseniya ................................... 1673

Shin, Sandra J..... 115, 136, 137, 199,

$225,226,227,1823$

Shin, Su-Jin ...........................254, 255

Shintaku, Itsushi P................. 1216, 1225

Shirai, Keisuke................................ 523

Shirsat, Hemlata .............................. 1994

Shiue, Yow-Ling ............................ 929

Shkedy, Sara ................................. 1996

Shnitsar, Iryna ............................... 1070

Sholl, Andrew B............................ 527

Sholl, Lynette M......................... 1162,1163 , $1212,1233,1242,1310,1809$, $1847,1926,1932,1934,1977$

Shon, Wonwoo .............. 504, 505, 536 Shows, Jared T .................. 1461, 1891

Shponka, Volodymyr.................... 1514

Shrestha, Rupendra ............. 450, 2085

Shrikhande, Shailesh.................... 1797

Shroff, Stuti G ...................... 709, 808

Shu, Catherine ................... 1928, 1965

Shui, Ruohong................................. 279

Shuja, Sania..................................... 452 


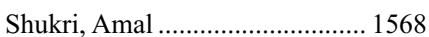

Shulman, Ira A ............................. 1564

Shyu, Susan.................................. 1995

Si, Qiusheng......... 406, 407, 485, 854

Siaghani, Parwiz ... 1515, 1518, 1545 ,

2102

Sibley, Richard .................. 1698, 1699

Sibony, Mathilde .......... 601, 848, 849

Sibulesky, Lena ............................. 1700

Sica, Gabriel....... 362, 784, 837, 1336

Sidawy, Mary .................................. 178

Siddiqi, Ahsan ................................... 85

Siddiqi, Anwer ............................... 452

Siddiqi, MA.................................... 707

Siddique, Ayesha .......................... 747

Siddiqui, Farzan ............................ 1340

Siddiqui, Javed .......... 497, 590, 1049,

1050,1069

Siddiqui, Momin T...... 104, 177, 216, $217,341,365,372,479,632$, $932,1299,1575,1657,2110$,

2143,2146

Siddiqui, Uzma ..................... 338, 415

Sidorenko, Tatyana.............. 842, 1766

Sidorov, Dmitry.............................. 1844

Sieber, Oliver ....................... 827, 828

Siefker-Radtke, Arlene.................. 883

Siegal, Gene P.... 169, 268, 296, 1313

Sienko, Anna ................................ 1183

Sierra, Adriana ............................ 1348

Sigel, Carlie.................................. 1773

Sigel, Keith .................. 406, 407, 485

Signori, Riccardo ........................ 1750

Sikora, Andrew G......................... 1314

Silva Morera, Carolina..... 1002, 1047

Silva, Elvio................................. 1093

Silva, Oscar ........................... 567, 1384

Silva, Thais.................................... 399

Silverman, Brittany ...................... 1812

Silverman, Jan F.......... 339, 376, 476, 926, 1285, 1815, 1822, 2098,

2111

Silvero, Arturo....... 1017, 1027, 1028,

1029

Sim, Jordan ....................... 453, 1048

Simard, Serge .................... 1940, 1975

Simi, Lisa ........................................... 46

Simko, Jeffry ..................................... 919

Simon, Marina................................. 1875

Simonyan, David.......................... 1962

Simpson, Jean F ................................ 266

Simpson, Roderick HW ............. 1282

Simsir, Aylin................ 343, 397, 1592

Sinard, John H............................. 2072

Singanamalli, Asha ....................... 1603

Singer, Bart ................................... 271

Singh, Arbind ................... 1752, 1991

Singh, Arjun ....................................... 35

Singh, Charanjeet.......................... 1955

Singh, Harsharan K........................ 1627

Singh, Kamaljeet.......... 176, 206, 247 ,

272

Singh, Kanchan ............................ 1819

Singh, Manmeet ............................ 2083

Singh, Naveena ............................... 1151

Singh, Rajendra................... 503, 1598

Singh, Rajesh ................................ 1022

Singh, Rajesh R....... 749, 1922, 2040

Singh, Varsha ............................... 1933

Singhi, Aatur D .......... 467, 806, 1813

Sinha, Namita................................. 807
Sinicrope, Frank A ……………...... 722

Siqueira, Sheila ................ 1450, 1451

Sir Philip, John............. 419, 420, 454

Sircar, Kanishka ................. 952, 1022

Siref, Andrew ................................. 536

Sirintrapun, Joe ....................... 404, 985

Sirintrapun, Joseph S ... 850, 916, 935

Sirintrapun, S Joseph .................. 1581

Sirintrapun, Sahussapont J .......... 874, $1037,1038,1053,1055,1058$

Sirohi, Deepika .................... 261, 892

Siroy, Alan E ........................ 525, 537

Sison, Cristina .................................. 800

Siziopikou, Kalliopi P......... 166, 288, $289,1329,1974$

Skala, Stephanie...... 497, 1049, 1050,

Skalova, Alena ........................... 1290

Skelly, Joan ..................................... 666

Skenderi, Faruk ...................... 69, 740

Skipper, Daniel C .......................... 273

Skrabek, Pamela............... 1483, 1519

Slade, Jamie ................ 419, 454, 490

Slanetz, Priscilla J .......................... 284

Slingluff, Craig L ................................. 539

Slivka, Adam....................... 467, 1813

Sloan, Emily A ................... 1241, 1263

Slodkowska, Elzbieta........... 231, 298

Slomovitz, Brian ......................... 1221

Small, David ............................... 2005

Small, Eric...................................... 934

Small, Thomas ....................... 661, 817

Smati-Grangeon, Sarra................... 601

Smith, Amber L.......... 455, 456, 1996

Smith, Joshua .................................. 732

Smith, Kelly ................................... 1558

Smith, Lauren................................. 1878

Smith, Stephen M.......................... 457

Smith, Steven C .... 16, 848, 892, 914, $954,1038,1050$

Smith, Theresa ............................. 2087

Smola, Brian ………………............ 401

Smyrk, Thomas .......... 722, 755, 1680

Sneige, Nour .................................. 224

Snezhkina, Anastasiya...... 1844, 1845

Snir, Olivia L................................ 308

Sniukiene, Vilma ......................... 1121

Snover, Dale C ................... 726, 1677

Snow, Anthony ....... 1301, 2023, 2088

Snow, Justin ................................ 2089

Snuderl, Matija... 48, 306, 1592, 1867

Snyder, Vivian S.......................... 1749

Snyderman, Carl H...................... 1318

Soares, Fernando.... 1027, 1028, 1029

Socec, Catherine I ........................ 1892

Soda, Masakazu .......................... 1955

Sohani, Aliyah........ 1363, 1547, 2059

Sojitra, Payal ................................ 1516

Sokol, Lubomir ............................. 1421

Solanki, Malvika ............................... 955

Solanki, Shraddha ......................... 867

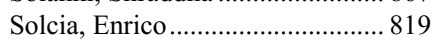

Soler, Teresa ......................................... 223

Soliman, Mahmoud L .................... 395

Soliman, Moaaz ........................... 1049

Solit, David ....... 850, 916, 985, 1055,

1058,1861

Solomides, Charlambos ............... 381

Solomon, David ............................. 1753

Solomon, Isaac.................. 1576, 1577

Solomon, James ................ 1051, 1754
Solorzano, Carmen C ..................... 477

Somerset, Hilary ............... 1297, 1331

Sominidi-Damodaran, Sindhuja... 506

Son, Jung Hoon ....................................... 1606

Son, Seung-Myoung ...................... 205

Sonawane, Shankar ...................... 2150

Sonawane, Snehal ......................... 2150

Sonett, Joshua .............................. 1928

Song, Buer.................................... 1644

Song, Hyung Geun........................ 205

Song, Jinming ......... 1469, 1517, 1551

Song, Joo..... 1483, 1515, 1518, 1519,

1545,2102

Song, Sharon …………………....... 808

Song, Weihua ................................. 1898

Soni, Shardul..................................... 1016

Sonkar, Abhinav A ....................... 1339

Sonmez, Ceyda ... 177, 218, 228, 229 ,

$\begin{array}{rr} & 274 \\ \text { Sono, Reiri } & 1377\end{array}$

Sood, Gagan ...................................... 1668

Soong, Richie..................... 813, 1196

Soong, T Rinda ................ 1242, 1243

Soong, Thing Rinda ...................... 950

Sorrells, Shelly ............................... 1477

Soslow, Robert .......1113, 1125, 1144, $1152,1201,1234,1244,1245$

Soto, Luis R .............................. 1520

Soubra, Ayman ................................ 991

Souers, Rhona J....... 464, 2068, 2069

Soukiazian, Armen........................ 287

Soukiazian, Nyrie............................ 287

Soumerai, Tara E.......................... 1144

Soundar, Esther ............................. 1524

Soundararajan, Suganthi ........... 1095,

Southey, Melissa ….......................... 207

Souza, Carolina A......................... 1904

Sowamber, Ramlogan ..................... 1137

Sowder, Aleksandra .................... 1337

Sozzi, Gabriella ........................ 595

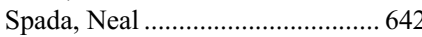

Spagnolo, Dominic V...................... 521

Spatz, Alan ..................................... 2005

Spaulding, Reed ............................. 150

Specht, Katja ................................. 1873

Spence, Jan....................... 1424, 2120

Spence, John ................................. 2120

Sperga, Maris .................................. 983

Spillane, Cathy ................... 1828, 1860

Spilseth, Benjamin ........................... 991

Sporn, Thomas A............................ 1901

Spratt, Daniel E................ 1049, 1069

Sprenkle, Preston ............................ 940

Springer, Simeon............................ 1018

Spruill, Laura …………………..... 954

Sriganeshan, Vathany ......................... 14

Srigley, John....................... 2090, 2091

Srinivasan, Ramaprasad............... 989

Srivastava, Amitabh .... 662, 667, 710,

833,1809

Srivastava, Shiv K....... 871, 872, 912

Srivastava, Supriya

Srougi, Miguel ................................... 959

St-Arnaud, Karl............................ 2125

St-Pierre, Catherine............ 2125, 2152

Staats, Paul........................... 398, 464

Stadler, Zsofia ................................ 732

Staerkel, Gregg A............................ 366

Staley, Charles................................. 787
Stallworth, Christina ..................... 1001

Stambolic, Vuk............................... 275

Stanek, Jerzy …………………..... 1172

Stanich, Peter ................................... 803

Stanoyevitch, Alexander ............. 1612

Starostik, Petr................... 1675, 1742

Starova, Blerta................................. 275

Stashek, Kristen M................ 709, 808

Stattin, Pär...................................... 896

Steele, Cindy ……………………... 422

Steele, Jane C .................................1113

Steele, Rebecca M........................ 2092

Stehlik, Jan ...................................... 849

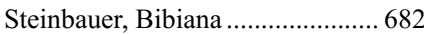

Steiner, David.................................. 688

Steiner, Petr ............................ 70, 983

Steinhilber, Julia............................ 1507

Steinhoff, Margaret M......... 247, 272,

Stelling, Adam............................. 17111

Stelow, Edward B.......................... 1927

Stem, Kodie........................................ 644

Stemmer-Rachamimov, Anat ..... 1763

Stemmer, Melissa ............................ 422

Stence, Aaron A......................... 54, 384

Stepanov, Oleg ............................ 1845

Stephens, Philip........... 196, 238, 496, 790, 994, 1005, 1015, 1138, 1297, 1331, 1654, 1836, 1855, $1880,1920,1924$

Stephenson, Philippe ...................... 458

Stevens, Todd ..................................... 95

Stevenson, Heather L ................... 1639

Stevenson, Margaret K...................... 86

Stewart, Colin J........ 521, 1189, 1251

Stewart, Delisha .............................. 193

Stewart, Douglas A....................... 1449

Stewart, Jimmie............. 418, 437, 484

Stewart, Jocelyn M...................... 1275

Stewart, John................................... 455

Stewart, Rachel L............................ 150

Stiles, Brendon M ............. 1937, 1938

Stinner, Jessica .............................. 1588

Stockley, Tracy............................ 1185

Stoffel, Michelle............................... 29

Stohr, Bradley .................................... 919

Stoler, Mark H................... 1208, 1209

Stolnicu, Simona ................ 1244, 1245

Stone, James R ................................ 320

Stoopler, Mark .................. 1928, 1965

Stram, Michelle........................ 459, 486

Straub, Melissa.......... 144, 251, 1246,

2082

Streutker, Catherine .............. 458, 691

Stricker, Phillip D ......................... 1603

Strickland, Kyle C....... 314, 607, 614,

1247

Strickland, Sarah V ..................... 1248

Strickland, Stephen A....... 1504, 1510

Striker, Gary E ............................ 1628

Strosberg, Jonathan R .................... 750

Stuart, Josh .............................................. 934

Stuart, Lauren N............... 2068, 2069

Stueck, Ashley E .......................... 1994

Sturgis, Charles D ....... 148, 149, 361,

$392,604,1322$

Sturkenboom, Kelli ......................... 550

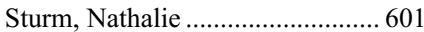

$\mathrm{Su}, \mathrm{Nan} . . . \ldots \ldots \ldots \ldots . . . . . . .1941,2116,2156$

Su, Shirley Y ................................ 1290

Su, Ting-Yi.................................... 1579 
Suarez, Adrian..........1192, 1602, 1611 Suarez, Yvelisse .......................... 1771 Sudhanshi, Jain ........................... 1869 Sugai, Tamotsu................................. 764 Suh, Hyerim ................................. 1333 Suh, James........... 196, 238, 496, 790, 994, 1005, 1015, 1138, 1297, $1331,1654,1836,1855,1880$, 1920,1924

Sui, Dawen .......................... 537, 1139

Sui, Long............................................. 465

Sukeda, Aoi .................................... 1954

Sukhanova, Madina...................... 1862

Sukhu, Balram............................... 1070

Sukov, William R .......... 74, 535, 689, 922, 990, 1086, 1971, 2008

Sullivan, Megan E......................... 101

Sulman, Erik P ............................. 1721

Sumathi, VP .................................... 87

Sumit, Isharwal .............................. 850

Sun, Belinda .................................... 809

Sun, Hongxia..................................... 460

Sun, Katherine............................... 1656

Sun, Lulu...................................... 2093

Sun, Tong …......................... 461, 1052

Sun, Wei ........................................... 343

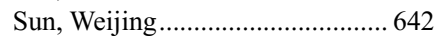

Sun, Xiangjie................................... 279

Sun, Yi............................ 1422, 1521

Sun, Yue ............................. 873, 1053

Sundar, Manonmani .......... 1777, 1785

Sunday, Mary E............................... 526

Sundin, Andrew........................... 1522

Sundling, Kaitlin ............................. 608

Sung, C James........... 247, 360, 1098, $1099,1205,1273$

Sung, Diana ......................................... 810

Sung, James C ................................ 176

Sung, Yun-Shao................... 33, 55, 56

Suñol, Mariona.................. 1409, 1893

Suo, Liye .......................................... 462

Suriawinata, Arief ....................... 1599

Surti, Urvashi ......... 1347, 1425, 1443

Suryo Rahmanto, Yohan ............. 1271

Susma, Alexandru ....................... 2150

Suster, David .................................. 1997

Suster, Saul.... 70, 521, 582, 848, 849, 983,1997

Sutton, Ann ...................................... 564

Suva, Mario..................................... 1763

Suzuki, Ayako ............................. 1701

Svensson, Maria ............................. 996

Swamy, Vinushree.............. 1466, 1523 ,

Swanson, Benjamin J.................. 1582

Swanson, Casey L ....................... 1270

Swanson, David ........ 56, 86, 88, 538,

2151

Swanson, Paul E........................... 1700

Swanson, PE 645

Swarrow, Rachel ................ 463, 1814

Swartwood, Steven ...................... 1891

Swee, Melissa ............................... 1645

Sweet, Joan ....................... 1008, 1070

Sweet, Kevin .................................... 791

Swerdlow, Steven H........ 1390, 1425, $1443,1458,1459,1540$

Swisher, Elizabeth............. 1226, 1248

Sy, Keiyan .................................... 2090

Syed, Jamil ........................................ 940

Syed, Sahr.

1616
Sykes, Lorraine .............................. 2144

Symmons, Martyn F.................... 1834

Szabo, Gyula .............................. 1829

Szafran, Adam T ............................ 114

Szankasi, Philippe ............. 1457, 1477

Szigeti, Reka ………………......... 1524

Tabatabai, Z Laura ................ 348, 464

Tabosa, Gabriel VBS.... 862, 863, 864

Tacha, David ................................ 1913

Tada, Toyohiro …................ 324, 1755

Tade, Funmilayo............................... 998

Taege, Alan.................................... 392

Tafe, Laura .......523, 1133, 1134, 1960

Taffurelli, Giovanni..................... 1780

Tagawa, Scott T........................... 1857

Taggart, Melissa ............................ 684

Taheri, Diana......... 1018, 1019, 1060,

1127

Taieb, Julien ………................... 779

Takahashi-Fujigasaki, Junko......... 617

Takahashi, Emiko......................... 1468

Takahashi, Hiroyuki .................... 1040

Takahashi, Yayoi ............................ 764

Takano, Masato .............................. 1632

Takhar, Mandeep........................... 1085

Talaat, Iman..................................... 152

Talamayan, Michael John C......... 276

Talat, Nadia .................................... 578

Talevich, Eric ......................... 199, 748

Talha, Rashid.............................. 1517

Talhouk, Aline.................. 1177, 1201

Taliano, Ross.................................. 747

Tallini, Giovanni ............................ 581, 615

Talman, Maj-Lis M ...................... 517

Talmon, Geoffrey ................ 565, 2124

Talwar, Aditya ............................... 1815

Tam, Simone ............................... 1151

Tam, Wayne............ 1349, 1440, 1463,

1465

Tamboli, Pheroze ..... 952, 1022, 1087

Tambouret, Rosemary H ... 549, 2059,

2078

Tamimi, Rulla ............................... 117

Tamm, Eric P.............................. 1785

Tamura, Daisuke .......................... 1249

Tan, Benjamin YC........................... 198

Tan, Benjamin Yongcheng ............ 277

Tan, Chih-Fen ................................ 350

Tan, Cong ....................................... 822

Tan, David SP................................ 1196

Tan, Dongfeng.......... 805, 1710, 2160

Tan, Lee K.................................... 165

Tan, Puay Hoon... 129, 134, 167, 184, $185,198,277,278,304,1840$

Tanaka, Kathryn E....................... 2016

Tanaka, Reiko................................... 58

Tanas Isikci, Ozlem...................... 1054

Tanas, Munir R............. 31, 53, 54, 89

Tang, Guilin .......... 1407, 1418, 1422, $1423,1441,1501,1521,1549$

Tang, Guillin ................................ 1446

Tang, Jean......................................... 518

Tang, Laura ................................. 1776

Tang, Ping .................................. 191, 287

Tang, Shangguo............................ 1213

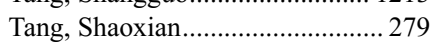

Tang, Yen Zhi ................................. 858

Tang, Zhenya..................... 1355, 1407

Tanguay, Jeffery J............................ 88

Taniguchi, Hiroyuki ..................... 1909
Tao, Xiang ........................................ 465

Taouli, Bachir............................... 843

Taplin, Mary-Ellen..... 873, 987, 1061

Tarabishy, Yaman ........ 679, 680, 780, $798,811,812,825$

Taraboanta, Catalin ..................... 1250

Tarrel, Robert F ............................ 888

Tas, Patrick.................................... 1444

Tashakori, Mehrnoosh................. 1525

Tashkandi, Hammad...................... 1756

Tateishi, Yoko................................ 2002

Tatevian, Nina ..................................... 27

Tateyama, Hisashi ........................... 1755

Taub, Robert..................... 1851, 1967

Taube, Janis M .... 43, 281, 282, 1707 ,

2003

Tavassoli, Fattaneh...... 239, 240, 241,

269

Tawfik, Ossama............. 189, 190, 431

Tay Kwang Yo, Timothy ............... 185

Taylor Smith, Paul J..................... 956

Taylor, Barry S .......... 850, 985, 1055 ,

1058

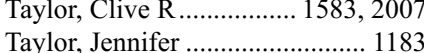

Taylor, William R........................... 755

Tayyari, Farnoosh........................... 280

Tazelaar, Henry D ...... 74, 1946, 2009

Teer, Jamie ..................................... 1230

Tefferi, Ayalew ………………….... 1554

Teh, Bin Tean ............. 277, 278, 1869

Teh, Ming ............................................ 813

Telatar, Milhan ............................... 492

Temprana-Salvador, Jordi .............. 1748

Temprana, Jordi............................. 890

Teng, Jiamin ...................... 1630, 2118

Teng, Kun-Yu ................................ 1696

Teng, Lianghong .......................... 1088

Teo, Min Yuen............................... 1055

Teo, Min-Yuen ................................ 985

Teo, Soo-Twang …………............... 207

Terada, Yukihiro................ 1182, 1249

Terasaki, Mika............................... 1943

Terasaki, Yasuhiro ........................ 1943

Terinte, Cristina................. 1244, 1245

Terra, Simone ................................. 942

Terra, Simone BSP .......... 1956, 1998,

1999

Terracciano, Luigi ........................ 1671

Terrones, Alexis B.......................... 492

Teruya-Feldstein, Julie................... 1377

Terzic, Tatjana ......... 466, 1208, 1251,

1252

Teshima, Christopher .................... 458

Tesic, Vera ......................................... 718

Tessier-Cloutier, Basile..... 814, 1201, $1253,1526,1787$

Têtu, Bernard ............................... 2091

Tetzlaff, Michael T...... 525, 533, 537, $1129,1379,1758,2018$

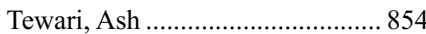

Tewari, Prerna ................................. 435

Thambudorai, Robin ........................ 794

Thapa, Prabin ................................ 921

Theise, Neil ........................... 555, 695

Theisen, Brian ................................. 467

Theparee, Talent ............................... 2075

Thiberville, Luc............................. 1973

Thiele, Juergen ............................... 1435

Thiene, Gaetano ................... 321, 322

Thike, Aye Aye ..... 184, 185, 198, 278
Thike, Daw Aye Aye....................... 277

Thirumala, Seshadri ........................ 544

Thivolet-Bejui, Françoise ........... 1950

Thomas, Brittany C ....................... 1270

Thomas, Daffyd G......................... 123

Thomas, Dafydd G........................ 2007

Thomas, George .............................. 934

Thomas, Geraldine ....................... 1320

Thomas, Kristen M ......................... 1867

Thomas, Lynita ………………..... 2063

Thomas, Rebecca ............................ 799

Thomas, Sarah C................... 815, 816

Thomas, Sumi ……………............. 1605

Thomason, Jon ...................... 89, 1781

Thompson, Elizabeth ... 146, 281, 282

Thompson, Lester DR ........... 75, 582,

1300,1338

Thompson, RH.............................. 922

Thompson, William............... 376, 926

Thomson, Brian............................... 518

Thomson, Timothy ........................ 154

Thorat, Swati............................... 1289

Thorn, Lindsay ............................ 1602

Thorner, Aaron ............................... 1803

Thorson, John A ................. 1561, 1754

Thrall, Michael J ............................ 440

Thung, Irene........................ 818, 1559

Thung, Swan N .......................... 1677

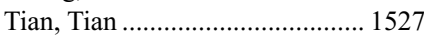

Tian, Wei ............................. 893, 1046

Tian, Xuejun.................................. 1528

Tickoo, Satish K.......... 850, 874, 916, 935, 985, 1037, 1038, 1053,

1055,1058

Tietz, Birgit ................................... 120

Tihan, Tarik ...................... 1727, 1753

Tilan, Jason ..................................... 1874

Timpe, Leslie ............................... 1865

Ting, David T ...................................... 631

Tinker, Anna................................. 1253

Tischkowitz, Marc ......................... 207

Tiska, Grace ................................... 1496

Tissier, Frederique............................. 601

Tiwari, Pallavi ................................. 1603

Tiwari, Sarita................................ 1603

Tiwari, Vandana ............................ 1339

Tixier, Lucie ........................ 248, 1107

Tjendra, Youley .............................. 1532

Tjionas, George A ………………....... 90

Todd, Nevins W ........................... 2015

Toffolatti, Luisa................................ 1750

Togashi, Miku ..................................... 764

Tokudome, Nahomi......................... 123

Toledo Dias, Ana B ……………... 1731

Tomaszewicz, Keith ......................... 461

Tomaszewski, John E....... 1341, 1644

Tominaga, Susumu........................ 1572

Tomlin, Bryan ............................ 1727

Tomlins, Scott A.......... 123, 882, 908, 909, 1038, 1050, 1056, 1068,

1069

Tondon, Rashmi …................ 709, 808

Toner, Mary.................................... 1326

Tong, Guo-Xia .............................. 571

Tong, Tommy ....................................... 1081

Tonooka, Akiko .................................. 71

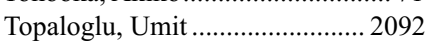

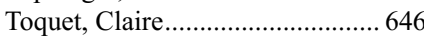

Torabi, Alireza....................... 91, 283 
Torbenson, Michael S ..... 1661, 1662, $1665,1666,1667,1671,1709$,

2084

Torii, Sho.......................................... 323

Torkko, Kathleen............................ 955

Torous, Vanda....... 121, 284, 468, 469

Torre, Matthew ............................ 1757

Torres-Cabala, Carlos A...... 525, 533, $537,1355,2018$

Torres-Mora, Jorge... 38, 75, 689, 900 Torres, Alba FC................ 1056, 1057 Torres, Joana ................................... 843 Torres, María M ................... 587, 1401

Torres, Mylin A ……...................... 208 Tosoian, Jeff........................ 859, 899

Tosoni, Antonella ........................... 879

Tosun, Akif B .............................. 1591

Toubaji, Antoun............................. 1058

Touijer, Karim A............................ 935

Toussaint, Andy.............................. 787

Toyokuni, Shinya ............................. 97

Tozbikian, Gary.............................. 300

Tracht, Jessica …............................... 470

Trahan, Sylvain ................. 1940, 1975

Train, Laura J ........................ 310, 311

Tran, Henry ................................... 1891

Tran, Hung ....................... 1823, 1852

Tran, Lily ……................. 1254, 1255

Tran, Thu..................................... 1034

Tran, Timothy............................... 2108

Tran, Tram.................................. 1658

Tran, Truc.................................... 1074

Travis, William...... 1896, 1903, 1910,

$1911,1953,2003$

Traylor, James G ........................ 2017

Treaba, Diana .................................. 540

Tregnago, Aline C ........... 1018, 1019,

$1059,1060,1127$

Trejo Bittar, Humberto..... 1949, 2000

Trepeta, Richard ............................... 75

Treseler, Patrick ........................... 1681

Tretiakova, Maria........ 966, 975, 984, 1061, 1062, 1066, 2071

Trevathan, Sean............................... 609

Trevino, Karen ......... 902, 1032, 1063

Trifilo, Katelyn E ........................ 2119

Trindade, Christopher J.... 1529, 1530

Trinh, Vincent Q........................... 2152

Tripathy, Debu................................... 147

Tripp, Sheryl …………………..... 1520

Trivedi, Anshu................................. 660

Trivella, Juan P............................. 1694

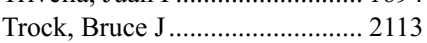

Troncoso, Patricia ......................... 1064

Trotter, Martin J ............................ 2091

Troxell, Megan...............111, 112, 676

Trpkov, Kiril...... 848, 849, 867, 1035,

1036,1065

Trudeau, Maureen ........................... 231

Trudel, Dominique ........................... 2106,

2125,2152

True, Lawrence ....... 934, 1014, 1061, 1062, 1066, 2071, 2080, 2137

Truong, Camtu D ........................ 1700

Truong, CD ......................................... 645

Truong, Duy .................................. 1996

Truskinovsky, Alexander M....... 1595

Tsai, Harrison ....................... 930, 1057

Tsai, Jen-Wei ....................... 92, 93, 94

Tsai, Jia-Huei ................................. 817

Tsang, Julia YS............................... 236
Tsao, Christin H ........................... 1531

Tsao, Ming-Sound........................ 1984

Tse, Gary M................................... 236

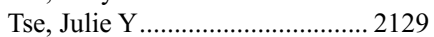

Tse, Ka Yu ................................... 1262

Tseng, Hsian-Rong......................... 2161

Tseng, Wiliam W............................... 67

Tsesmetzis, Nikolaos........ 1354, 1492

Tsokos, Maria.................................... 85

Tsongalis, Gregory J ........... 523, 769,

1960,2129

Tsuchikawa, Takahiro ................. 1817

Tsujimura, Tohru ........................... 1942

Tsung, Allan .................................. 1813

Tsuzuki, Toyonori ........................ 1067

Tu, Shi-MIng................................ 1087

Tu, Xiaoyu ......................................1111

Tuchiya, Tomoshi......................... 1958

Tull, Jamie....................................... 529

Tuluc, Madalina ........................... 1952

Tumas, Jordyn B ......................... 1894

Turashvili, Gulisa.................. 285, 286

Turbat-Herrera, Elba A....... 487, 1630

Turcotte, Stéphane ...................... 1925

Turk, Andrew T ............................... 582

Turner, Bradley M................ 191, 287

Tutaeva, Victoria ............................. 197

Tuttle, Kimberly .................... 258, 259

Tuttle, R Michael ..........581, 615, 616

Twaddell, William........................... 766

Twardowski, Przemyslaw ........... 1081

Tyekucheva, Svitlana .................... 897

Uamg, Huaitao .............................. 488

Ubago, Julianne M ................ 288, 289

Uccella, Silvia ................................. 570

Uchida, Katsunori ......................... 1816

Uchiyama, Tomoko....................... 1632

Udager, Aaron ........... 590, 1068, 1069

Udager, Aaron M....... 908, 909, 1049,

Udo, Emiko ................................... 2001

Uehara, Takeshi................... 758, 1794

Ueki, Nozomi................................ 2142

Ueno, Takashi................................ 1817

Uesugi, Noriyuki............................ 764

Ugalde, Paula ................................ 1975

Ulamec, Monika............... 1054, 1065

Ulbright, Thomas M.... 561, 902, 974, 1010, 1032, 1063, 1124, 1939

Ullman, David.................................. 95

Umar, Beena......................................... 1340

Umeda, Shigeaki ......................... 2002

Umetsu, Sarah E......... 37, 1663, 1829

Umphress, Brandon.......... 1256, 1257

Underwood, Dawn .......................... 392

Unger, Pamela ............................... 1032

Unitt, Christine............................ 1528

Uniya, Upasana ............................... 516

Untanu, Ramona ............................ 471

Uram-Tuculescu, Cora .................. 914, 1232,

Urken, Mark L............................... 582

Uruga, Hironori............................... 1993

Ushiku, Tetsuo ............................... 662

Ussakli, Cigdem .............................. 966

Uto, Kenta ..................................... 317

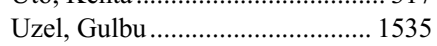

Vachharajani, Neeta ......... 1660, 1695

Vadlamudi, Kumari ......................... 499

Vafaii, Pardis ................................... 290

Vaghasia, Ajay................................. 853
Vaghefi, Amineh..

1532

Vaickus, Louis J ............................ 430

Vail, Paris J................. 100, 237, 809

Vaiyapuri, Sumathi.................... 55, 56

Vakar-Lopez, Funda .......... 1066, 2071

Vakiani, Efsevia .......... 668, 732, 733,

1861

Valasek, Mark A........ 788, 818, 1559

1561

Valbuena, Jose R ........................... 1533

Valderrabano, Pablo ......................... 603

Valencia-Cedillo, Raquel ............ 1101

Valent, Alexander .............................. 473

Valentino, Alessandro .................. 1923

Valera, Alexandra ......................... 1475

Valesini, Guido.............................. 1723

Valle, Juan ........................................ 762

Vallejo-Benítez, Ana ......................... 1030

Vallone, Joseph ………………..... 1387

Valluru, Neelima .... 1578, 1704, 1826

Valori, Laura ............................... 1750

Van Arnam, John............... 1665, 1758

Van Cott, Elizabeth M................. 1454

van de Rijn, Matt................... 41, 1832

Van de Vijver, Koen ....................... 1094

van der Kwast, Theodorus ........ 1008,

1009,1070

Van Dyke, Alison ............. 1774, 1795

Van Dyke, Daniel L...................... 2147

van Kempen, Leon C ...................... 2005

Van Keulen, Virginia P................. 1956

Van Straten, Marta ...................... 2094

Van Vrancken, Michael ........ 633, 636

Van Zante, Annemieke .................. 199

Van Ziffle, Jessica ......................... 1753

VanBeek, Christine ..................... 1623

VandenBuscche, Christopher J..... 402

Vandenbussche, Christopher ....... 375,

$390,1772,2066$

VanderLaan, Paul .................. 468, 469

Vanecek, Tomas .................... 520, 522

Vanel, Daniel............................ 47, 83

Vang, Russell............ 297, 1127, 1271

Vankalakunti, Mahesha ................. 892

Vanoli, Alessandro .............. 819, 1782

Varadan, Vinay .................................. 160

Varadhachary, Gauri..................... 1777

Varambally, Sooryanarayana....... 1406

Vardaki, Ioulia................................ 1492

Varesano, Serena .......................... 1923

Varga, Zsuzsanna .................. 242, 291

Vargas, Cindy .................................. 303

Vargas, Sara.................................... 1757

Varghese, Sharlin .......................... 2095

Varma, Kavita ……….................... 1258

Varma, Kavita R................. 209, 1103

Varma, Sonal...... 245, 256, 257, 2145

Varma, Sushama............................... 676

Varma, Vishal K ............................. 2104

Varney, Ruan C ................................. 2096

Varshney, Neha .............................. 292

Vasa, Puneeta S .............................. 472

Vasef, Mohammad ....................... 1930

Vasiliadis, Theodore....................... 312

Vasiljevic, Alexandre .................. 1726

Vasmatzis, George....... 922, 942, 990,

1956

Vassallo, Robert ……………….... 2014

Vavinskaya, Vera ............................. 818, 1559

Vaz, Fátima …………………….... 249

Vazquez, Ivonne ........................... 1475
Vega, Francisco ......... 59, 1369, 1431, 1532

Vega, Sandra ....................................587

Veillon, Diana M........................... 2017

Veits, Lothar........................................ 679

Velazquez Vega, Jose E... 1737, 1759 ,

1760

Velcheti, Vamsidar ............................ 361

Velcheti, Vamsidhar ........................ 1607

Velez, Moises J.............................. 2003

Velloso, Elvira.................... 1450, 1451

Veloza, Luis..................................... 1534

Venick, Robert S .......................... 1713

Venkataraman, Girish.......... 512, 1623

Vennapusa, Bharathi .................. 2114

Venta, Luz A................................... 224

Veremis, Brandon......................... 1590

Vergilio, Jo-Anne .................. 196, 238, 496, 790, 994, 1005, 1015, 1138, 1297, 1331, 1654, 1836, 1855, $1880,1920,1924$

Verhoest, Gregory ........ 946, 947, 948

Verma, Amit .................................. 1394

Verma, Anuj ..................................... 634

Verstak, Brett................................. 1834

Vey, Brianna L................................ 911

Viaene, Angela ................................... 2097

Viala, Sandrine .............................. 1107

Vidal, August................................ 1229

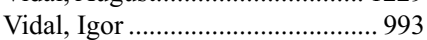

Vielh, Philippe................................... 473

Vierkoetter, Koah .....1119, 1120, 1259

Vierling, John ............................... 1668

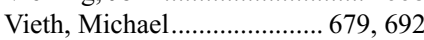

Vignali, Marissa ............................... 1493

Vij, Kiran R................................. 1350

Vikneswari, Rajasegaran................. 278

Vila Duckworth, Lizette............... 1675

Vila, Jordi ....................................... 1573

Vilar Sanchez, Eduardo.................. 684

Vilaseca, Isabel .................................. 359

Villada, Gabriel ............................. 1369

Villalobos, Pamela ....................... 1193,1194

2153

Villanueva, Alberto ....................... 1229

Villaseca, Miguel .... 784, 1774, 1795,

1795,
1804

Villaseca, Miguel Angel............... 1808

Vinayak, Shaveta............................. 160

Vincentelli, Cristina ......................... 14

Vinco, Michelle L................................ 590

Virk, Sohaib ................................. 1333

Virmani, Renu .................................. 323

Visscher, Daniel W...... 139, 175, 264,

2094

Viswanath, Satish .......................... 1603

Viswanatha, David S....... 1397, 1512,

1554

Vitkovski, Taisia.................... 293, 799

Vivallo, Carolina ............................... 1774

Vivero, Marina ............ 441, 474, 1977

Vizcaino, Maria Adelita ............... 1761

Vladoiu, Maria C .............. 2125, 2152

Vladoiu, Maria-Claudia ............... 2106

Vlassara, Helen ............................... 1628

Vnencak-Jones, Cindy L............ 1504,

1510

Vogel, Ashley N .......................... 1894

Vogel, Hannes ............................... 1124

Vogel, Rachel I................................ 450

Vogel, Victor G ............................. 197 
Vogelstein, Bert.............................. 1018

Vohra, Poonam............................... 348

Volchenko, Nadezhda.................. 1844

Volmar, Keith E................. 2068, 2069

Volpato, Anna G......................... 1750

Voltaggio, Lysandra .... 680, 701, 780,

$781,798,806,811,812,825$

Volyanskyy, Konstantin ... 1025, 1646

Volz, Marcus R............................... 510

von Deimling, Andreas .................... 81

von Rundstedt, Friedrich-Carl ..... 976

vonRentzell, Jeanne E................. 2020

Vora, Moiz.................................. 2105, 2154

Vora, Tushar ..................................... 80

Vose, Julie M................................ 1527

Vosoughi, Aram............................... 475

Voss, Jesse S.................................. 2010

Voss, Martin H ................................ 874

Vrana, Julie A ................................ 2004

Vranic, Semir ............... 69, 740, 1989

Vuong, Huy G ................................. 610

Vyas, Monika ....................... 294, 820

Vytlacil, Christopher .......... 476, 2098

Wachsmann, Megan ..................... 1818

Wachtel, Mitchell S............................ 544

Wade, Scott L .................................. 1611

Wadile, Amol ................................. 1762

Wagner, Siegfried.......................... 1914

Wajapeyee, Narendra .................... 294

Wake, Laura ................................... 1535

Wakely, Paul E ........... 386, 457, 1309

Wala, Samantha.............................. 891

Walavalkar, Vighnesh............ 172, 174

Wald, Abigail ............................. 1730

Walia, Saloni .................... 1260, 1261

Walker, Kimberly...... 188, 510, 1168 ,

1367

Walker, Neal................................. 728

Walkovich, Kelly ......................... 1878

Wall, Carolyn M.......................... 2130

Wallace, Dean ............................... 1903

Walsh, Edward G ......................... 2135

Walsh, Joanna................................ 2042

Walsh, Joanna C............................. 566

Walsh, Michael................. 2104, 2148

Walter, Matthew ................ 1350, 1496

Walter, Vonn ................................... 612

Walts, Ann E ...................... 611, 1980

Waluszko, Aneta....... 842, 1317, 1766

Wang-Miller, Su ........................... 1405

Wang, Alun R ................................. 527

Wang, Anna Y ............................ 2005

Wang, Ariel SY ............................. 1262

Wang, Beverly Y .......................... 2065

Wang, Chen .................................... 1278

Wang, Dehua .................................. 1892

Wang, Dennis ............................... 1039

Wang, Endi.............. 1442, 1447, 1886

Wang, Gang.................................. 1071

Wang, Guiqing ..................... 1563, 1565

Wang, Guisong .............................. 1261

Wang, Hai............................... 295, 831

Wang, Hangjun.............................. 2005

Wang, Hanlin L......... 657, 726, 1682,

1703, 1711, 1713

Wang, Hao-Wei ............................. 1536

Wang, He........... 312, 336, 411, 1327 ,

1993

Wang, Helen H............................... 358

Wang, Hua...................................... 1777

Wang, Huamin .................. 1777, 1785
Wang, Huan-You

1947

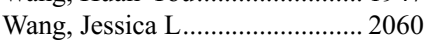

Wang, Jianfu.................... 2128, 2155

Wang, Jindong...................... 336, 411

Wang, Jinhui..................... 1081, 1519

Wang, Kai......... 296, 821, 1297, 1331

Wang, Lai Mun .............................. 712

Wang, Larry ................................. 1712

Wang, Lei ...................................... 822

Wang, Li.......................................... 465

Wang, Li J ................... 747, 777, 977

Wang, Li Juan ................... 1916, 1917

Wang, Li-Chong ............................ 2116

Wang, Li-Ping .............................. 709

Wang, Lin..................................... 1552

Wang, Linlin...................... 1351, 1705

Wang, Lu............... 65, 79, 443, 2123

Wang, Lynn Y............................... 1862

Wang, Michael .............................. 1500

Wang, Mingsheng ....................... 1031

Wang, Minhua ................... 1341, 1345

Wang, Minjing ............................. 1537

Wang, Peng .................................. 2099

Wang, Sa ..... 1384, 1403, 1418, 1422 , $1434,1441,1452,1521,1539$

Wang, Shu-Juan ......................... 1538

Wang, Tian-Li ................... 1238, 1271

Wang, Ting Ting.......................... 813

Wang, Wei ... 1353, 1357, 1373, 1374,

$1407,1408,1423,1539$

Wang, Wei-Lien .... 32, 41, 45, 72, 92,

93, 94, 455, 500, 533, 1193, 1194

Wang, Weige ................................ 1862

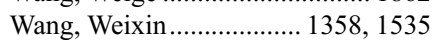

Wang, Xiangxue............................ 1607

Wang, Xiao-Ming M.................... 2156

Wang, Xiao-Ming Mindy........... 1941,

Wang, Xiaofei ..................... 704, 2087

Wang, Xiaoyan................................. 442

Wang, Xuan J ............................... 1540

Wang, Xuemei................................. 1064

Wang, Yan ..................................... 1278

Wang, Yanhua ........ 1394, 1747, 1839

Wang, Yao ......................................... 499

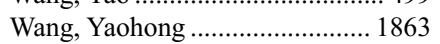

Wang, Yihong...... 176, 272, 295, 700,

1273

Wang, Ying............. 1000, 1072, 1073

Wang, Yiying.................................... 542

Wang, Yu-Chen ....................... 823, 824

Wang, Yuxuan .............................. 1018

Wang, Zeng-Feng ................................. 501

Wang, Zengfeng ............................. 702

Wang, Zhen .................................... 1415

Wang, Zhenglong .......................... 493

Wani, Khalida......... 45, 72, 92, 93, 94

Ward, Heather ............................. 1516

Ward, Kevin C................................. 208

Ward, Mark ………............................. 1828

Ward, Nicholas D......................... 1647

Ward, Stephen C .... 1631, 1653, 1691

Warf, M Bryan .............................. 518

Wargo, Jennifer A............................ 533

Warlick, Christopher ...................... 991

Warmke, Laura.............................. 1232

Warnecke, Gregor ........................... 1945

Warnke, Roger ................. 1390, 1472

Warren, Christine B...................... 1744

Warren, Daniel ................................ 122
Warrick, Joshua I....... 612, 613, 1074, 1342

Wartenberg, Martin ...................... 1788

Wasag, Bartosz................................ 501

Wasco, Matthew J ............... 551, 1077

Wasfy, Rania E............................ 1751

Washburn, Erik R................... 347, 613

Washington, Kay............................. 687

Wasserman, Allison M ................. 1388

Watanabe, Shun-ichi ................... 1954

Waters, Kevin...... 325, 679, 680, 780, $798,811,812,825,1706$

Watkin, William .................. 101, 2075

Watkins, Jaclyn C................ 314, 1931

Waxman, Irving.....................338, 415

Weaver, Donald L............. 2032, 2034

Webb, Milad.................................. 1541

Weber, Christopher ............... 628, 629

Wei, Bo......................................... 274

Wei, Christina................................... 1075,1564

Wei, Dongguang................................ 96

Wei, Eric X.................................... 2154

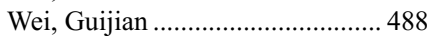

Wei, Jian-Jun ................................ 1257

Wei, John........................... 909, 1068

Wei, Shi......... 95, 169, 268, 296, 893

Weichert, Wilko ............................ 1791

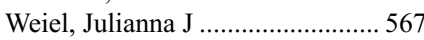

Weigelin, Helmut ............................ 868

Weigelt, Britta ..... 161, 207, 220, 242, $243,1152,1312,1854$

Weihua, Huang.............................. 1871

Weinberg, Olga K... 1439, 1542, 1543

Weinreb, Ilan...................... 1294, 1312

Weinreb, Jeffrey .............................. 940

Weinstein, John N ......................... 883

Weir, Michele M .......566, 1153, 1154

Weisenburger, Dennis D ........... 1483, $1515,1518,1519,1545,2102$

Weiser, Martin..

Weisman, Paul............................... 1232

Weiss, Lawrence ............................. 1363

Weiss, Ronald L ............................ 2067

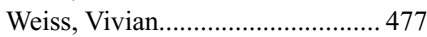

Weissferdt, Annikka .................... 2006

Weizer, Alon Z ................... 1049, 1069

Weksler, Benny ............................. 1827

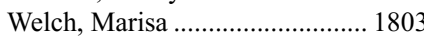

Welder, Daniel.................................... 283

Wellmann, Axel........................... 2157

Welsh, Cynthia .................................. 1719

Wemmer, Megan .......................... 2121

Wen, Hannah Y ... 161, 207, 242, 285 ,

Wen, Kwun Wah

Wen, Shuai ...................................... 2159

Wendy, Wong .................................. 232

Wenger, Doris ................................... 39

Wenig, Bruce M ............................. 582

Wenstrup, Richard.............................. 518

Wertheim, Gerald .......................... 1544

Wessel, Irene .................................. 1284

West, Rob ............................................ 676

West, Robert

676

Westaby, Joseph ....................326, 327

Westerhoff, Maria .... 726, 1656, 1673

Westra, William H... 436, 1057, 1282 , $1291,1292,1302,1979$

Wey, Elizabeth ........................... 1548

Weynand, Birgit .............................. 478

Wheelan, Sarah J

.... 478

White, Bartholomew ..................... 1342

White, Charles L ......................... 1725

White, Christine ............................ 435

White, Kristie L.................. 546, 2029

White, Marissa ............................... 297

Whitehall, Vicki ............................... 728

Whitington, Thomas...................... 895

Whitman, Jeffrey.......................... 2029

Whitney-Miller, Christa L............. 804

Whitney, Jon ....................... 187, 1864

Wi, Youngchan...................... 254, 255

Wians, Frank H ...................... 91, 283

Wick, Mark R................................ 2133

Wick, Myra J............................... 1270

Wieck, Lucas................................... 479

Wiesner, Georgia L ...................... 1246

Wietlisbach, Larissa ..................... 1874

Wiggins, Charles ........................... 1293

Wiklund, Peter ................................ 895

Wilcox, Ryan................................. 1460

Wild, Peter ...................................... 291

Wiles, Walter G............................... 998

Wiley, Clayton................................ 1587

Wiley, Elizabeth ....... 262, 1179, 2083

Wilkerson, Myra L ................ 964, 965

Wilkes, David C................................ 77

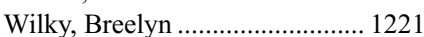

William, Christopher.................... 1592

Williams, David .................... 827, 828

Williams, Dawn ............................... 2100

Williams, Erik ............................... 1763

Williams, Grant................................ 1076

Williams, JoAnna ......................... 1602

Williams, Marcus D ........................ 547

Williams, Michelle D......... 455, 1728

Williams, Nicole .............................. 160

Williams, Nicole C................ 612, 613

Williams, Phillip ………………... 2158

Williams, Roy ............................. 1918

Williamson, Sean R... 551, 943, 1044 , $1065,1077,1078$

Willis, Brian C ........ 539, 1241, 1263

Willis, Eric ....................................... 829

Wilson, Christine ......................... 2035

Wilson, Julie M ............................ 1835

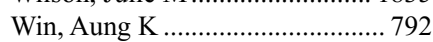

Winer, Daniel ................................... 579

Winer, Joshua H ..................................... 787

Winnepenninckx, Véronique......... 509

Winship, Ingrid .................... 791, 792

Winton, Elliott................................. 1550

Wistuba, Ignacio I....... 125, 513, 749,

$1193,1194,2153$

Wiszowaty, Alicja ............... 285, 286

Witkiewicz, Agnieszka K.... 100, 237,

809

Witt, Benjamin L....... 405, 464, 1337

Witte, Owen ................................ 934

Wobker, Sara E........... 866, 953, 1079

Woda, Bruce A ............................... 451

Wojcik, Eva M .... 333, 427, 428, 443,

$444,445,957$

Wolak, Emily …............................. 25

Wolber, Robert ............................. 2091

Wolfe, Jenna ...................................... 425

Wolff, Daynna J .................. 273, 1719

Wolff, Robert................................. 1777

Wollison, Bruce.............................. 1803

Wolpin, Brian ................................... 1803

Wolsky, Rebecca J........................ 1264

Wong, Adele........... 1265, 1266, 2101 
Wong, Andrew ........ 86, 88, 538, 2151 Wong, Jerry T.................... 1545, 2102 Wong, Kristine S ............................ 614 Wong, Mary ................ 568, 830, 1608 Wong, Meng Kang ...................... 1869 Wong, Oscar GW ......................... 1262 Wong, Richard .................. 1080, 1865 Wong, Serena ........... 133, 1267, 1281 Wong, Siew-cheng ......................... 184 Wong, Waihay J ............................ 1546

Wong, Willard .................................. 298

Wood, Brent L............................. 1529

Wood, Christopher G .................... 952

Wood, Laura D................... 761, 1688

Wood, Simon................ 884, 885, 886

Wood, Stephanie ........................... 831

Woods, Jennifer N......................... 482

Woolf, Nicholas .............................. 832

Wooten, Melanie ………………..... 1648

Wozniak, Laura J................ 690, 1713

Wrana, Jeffrey L......................... 1070

Wu, Angela...... 908, 909, 1012, 1068, 1764

Wu, Ashley ................................... 1727

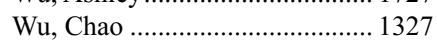

Wu, Elizabeth Yiru.............. 833, 1268

Wu, Erxi ........................................... 102

$\mathrm{Wu}, \mathrm{H} \ldots \ldots \ldots \ldots \ldots \ldots \ldots \ldots \ldots \ldots \ldots \ldots \ldots \ldots \ldots . . .1306$

Wu, Howard ....... 353, 442, 480, 1898

Wu, Huiqing ................................ 1081

$\mathrm{Wu}$, Natalie ................................... 232

Wu, Peter........................................ 1867

Wu, Richard ................................ 2159

Wu, Sharon....................................... 396

$\mathrm{Wu}$, Tao ………………………...... 1269

Wu, Tongtong........................623, 805

Wu, Tsung-Teh ............ 689, 721, 754, $1661,1666,1667$

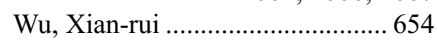

Wu, Xingyong .................. 2116, 2156

Wu, Xinyu .............................299, 834

Wu, Xiwei .......................... 1081, 1519

Wu, Xuemei .................................. 1270

Wu, Yadi.......................................... 150

Wu, Yiru ........................................ 1803

Wu, Yun.................... 301, 1846, 2128

Wurst, Michelle N.......................... 629

Wyatt, Alexander W ....................... 930

Wyrzykowski, Mary..................... 1548

Xess, Immaculata ......................... 1566

Xia, Daniel .................................... 1547

Xia, Rong ..................................... 1609

Xia, Tian.......................................... 835

Xian, Rena R ................................ 2054

Xian, Zhaoying ………………......... 300

Xiao, Guang Q ....... 1010, 1032, 1075

Xiao, Hong................................... 1050

Xiao, Li ................................................... 1071

Xiao, Shu-Yuan... 338, 628, 629, 654,

$718,719,1656$

Xiao, Xiuli........................................ 835

Xiao, Yaoxing...................................1111

Xie, Catherine ................................ 1548

Xie, Hao ........................................... 654

Xie, Ming ..................................... 1548

Xie, Steve ........................................ 725

Xin, Wei ............................... 640, 1779

Xing, Deyin............ 1232, 1271, 1272

Xing, Dongmei.................... 836, 1707

Xing, Juan ......................................... 356

Xiong, Jinjun................................ 1273
Xiong, Wei ..................................... 1295

Xiong, Yin.......... 50, 603, 1843, 2038

$\mathrm{Xu}$, Amanda Shuo ............... 256, 2145

Xu, Bin...... 447, 581, 615, 616, 1294 ,

1343

$\mathrm{Xu}, \mathrm{Bo}$................................ 875, 1082

$\mathrm{Xu}$, Haiying ............................. 43, 282

Xu, Haodong ................................... 1988

$\mathrm{Xu}$, Huihong.................................. 395, 832

Xu, Jia ............................ 1649, 2019

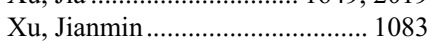

$\mathrm{Xu}, \mathrm{Jie}$.............................. 1441, 1549

Xu, Mina ......................................... 1359

$\mathrm{Xu}$, Mina L................................... 1481

$\mathrm{Xu}$, Ruliang .................................... 647

Xu, Wei .......................................... 1895

$\mathrm{Xu}$, Xiaoli....................................... 279

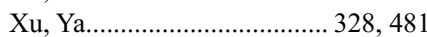

Xu, Yan...........................................1111

$\mathrm{Xu}$, Yayun....................................... 845

$\mathrm{Xu}$, Zhaolin ............................... 1994

Xue, Yue...... 671, 714, 756, 787, 837, $1795,1799,1800$

Yaar, Ron...................................... 503

Yab, Tracy C.................................... 755

Yacoub, Joseph.................... 936, 937

Yacoub, Mokrane .......... 946, 947, 948

Yagi, Yukako ................... 1585, 1992

Yakirevich, Evgeny ............... 540, 977

Yamada, Shozo................................. 617

Yamada, Yuichi ................................ 97

Yamaguchi, Yuka ........................ 2142

Yamamoto, Hidetaka..................... 838

Yamanaka, Kazuki ....................... 1935

Yamasaki, Naoya......................... 1958

Yamashita, Kyoko .............................. 97

Yan, Jie.......................................... 1365

Yan, Li................................ 210, 211

Yan, Shaofeng ...................... 531, 532

Yan, Ying...................................... 1527

Yan, Zhen ................................... 1708

Yang, Bin............................ 1100, 1280

Yang, Chen..... 839, 1279, 1346, 1650

Yang, Cheng-Han........................ 1579

Yang, Chi-Shun .............................. 480

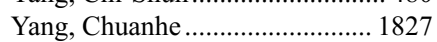

Yang, David....................... 1479, 2117

Yang, Dongfang ............................ 1917

Yang, Fei ............................... 125, 301

Yang, Fusheng.............................. 1712

Yang, George.................................. 2107

Yang, Guang-Yu.... 15, 577, 635, 674,

$711,1842,2036$

Yang, Haichun............................... 1651

Yang, Hong ................................... 1445

Yang, Huaitao................................. 489

Yang, Hui-Min ..................... 723, 783

Yang, Jing...................................... 208

Yang, Kaixuan................................ 1170

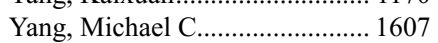

Yang, Michelle X ................... 665, 666

Yang, Minnan................................. 835

Yang, Ping .................................... 1956

Yang, Qi ........................... 622, 1075

Yang, Shi......................................... 503

Yang, Soo-Ryum ............................. 371

Yang, Tong ..... 932, 1550, 1759, 2146

Yang, Wei ......................................... 472

Yang, Wentao .......................279, 1111

Yang, Ximing J.................... 924, 2123

Yang, Yang ................................. 1406
Yang, Zhaohai .

Yang, Zhiming

Yang, Zhongbo. .

Yantiss, Rhonda K....... 669, 696, 782 ,

836,1807

Yao, Keluo............... 1602, 1610, 1611

Yao, Song .............................. 210, 211

Yao, Takashi .................................... 317

Yasir, Saba........................ 1680, 1709

Yassan, Lindsay............. 483, 628, 629

Yassen, Noha N ............................... 99

Yates, Jennifer .................................. 451

Yazdani, Maryam ............................. 123

Yazigi, Nada............................... 1895

Yaziji, Hadi ........................ 303, 2007

Ye, Fei .... 541, 842, 1198, 1317, 1766

Ye, Huihui ........................... 873, 987

Yearsley, Martha ............................ 803

Yegnasubramanian, Srinivasan .... 853

Yeh, Chen.

.. 1313

Yeh, Iwei ................................ 199, 748

Yeh, Matthew M..... 1659, 1661, 1700

Yeh, MM ............................ 645, 1677

Yemelyanova, Anna ..........1193, 1194

2153

Yen, Ten-Yang ............................. 1865

Yeoh, Khay Guan ............................ 813

Yeong, Joe.......... 184, 185, 304, 1840

Yi, Chun-Hui................................... 541

Yi, Eunhee S.......... 1915, 1946, 1947, 1956, 1971, 2004, 2008, 2009, 2010, 2013, 2014

Yilmaz, Asli.... 867, 1035, 1036, 1065 Yilmaz, Helene .................. 1065, 1085

Yin, C Cameron ……................... 1500

Yin, Cameron ......... 1402, 1416, 1441, 1549

Yin, Changhong ...... 924, 1023, 1563 ,

Yin, Minzhi ................................. 1869

Yin, $\mathrm{Yu}$.......................................... 934

Yin, Zichang..................... 1710, 2160

Yip, Linwah.................................... 425

Yoder, Sean ................................... 1230

Yoest, Jennifer............................... 2103

Yokomise, Hiroyasu ......................... 1936

Yong, William ............................ 1753

Yoon, Esther C ...... 1274, 1563, 1697 ,

1732,1765

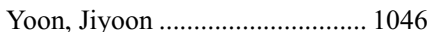

Yoon, Ju-Yoon................................ 1275

Yoon, Sun Och ............................ 1296

Yoon, Yoo-Seok ……………….... 1792

Yorozu, Takashi............................ 1040

Yoshida, Akihiko........................... 71, 98

Yoshida, Makiko ......................... 1632

Yoshida, Megumi ........................ 1755

Yoshimoto, Toyoki .......................... 617

Yoshimura, Masayo...................... 2011

Yoshiura, Koh-ichiro....................... 2142

Yoshizawa, Akihiko ........ 1899, 1957,

1993

Yoshizawa, Saeko ........................ 317

Yotsukura, Masaya ....................... 1954

You, Jia.............................................. 489

You, Xiaoming .......................635, 674

Younes, Mamoun ................. 630, 1819

Younes, Pamela S........................ 1819

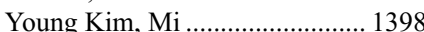

Young, Ken H.............................. 1422

Young, Neal S
Youngren, Jack ................................. 934

Youngson, Bruce .......................... 2035

Younke, Brianne................................ 27

Yousaf, Hira ......................... 449, 606

Yousef, George M...... 891, 963, 1024

Yousefi, Kasra ........... 860, 1056, 1085

Yousefi, Safoora ........................... 1760

Yousem, Samuel A............ 1589, 1949

Yozu, Masato................................... 662

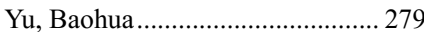

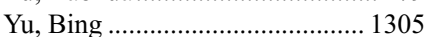

Yu, Eunsil..................................... 1790

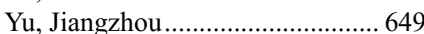

Yu, Jing ……………………....... 1258

Yu, Jun............................................ 761

Yu, Lianbo.................................... 1696

Yu, Wengdong ................................. 1668

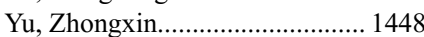

Yuan, Chen ....................................... 1803

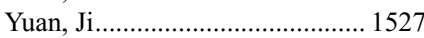

Yuan, Liping................................ 1222

Yuan, Songyang ................................ 743

Yuan, Wei ....................................... 2107

Yubero-Serrano, Elena M............ 1628

Yucel, Recai .......................................... 1919

Yue, Changjun............................... 1482

Yue, Ming....................................... 1370

Yue, Yong ............................. 107, 108

Yuh, Bertram ................................. 1081

Yung, Evan........................................... 363

Yusuf, Yasmin ................................ 1697

Yuwono Tjota, Melissa .................. 1980

Zaccarini, Daniel.......................... 1344

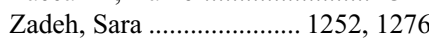

Zafar, Nadeem.............................. 1775

Zafar, Rabia..................................... 192

Zaidan, Bruna C ............. 862, 863, 864

Zaidi, Tanweer M....................... 1996

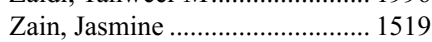

Zaloudek, Charles .......................... 199

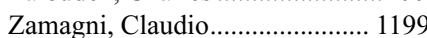

Zamboni, Giuseppe....................... 1773

Zamecnik, Michal .......................... 522

Zanatta, Lucia ............................... 1750

Zander, Dani S ............................. 2057

Zanetti, Maurizio.................................. 1051

Zannoni, Gian Franco ................. 1277

Zapata-Tarres, Marta..................... 1868

Zarbo, Richard ..................................... 2096

Zarei, Shabnam ........................... 1278

Zaretsky, Andrew ............................. 1845

Zargham, Ramin.......................... 1086

Zarrin-Khameh, Neda .................. 421, 481

Zarzour, Jessica ............................... 918

Zavodszky, Maria............................ 163

Zeck, Jay ....................................... 178

Zeh, Herbert J...................... 467, 1813

Zehir, Ahmet .... 165, 850, 1831, 1837

Zehnder, James........ 688, 1437, 1471,

Zehrouni, Nourredine............ 507, 508

Zeng, Janice ................................. 2090

Zeng, Jennifer ......305, 306, 307, 1116

Zeng, $\mathrm{Xu}$..................................... 3, 5

Zeng, Zhengyu ..................................... 1269

Zeppernick, Felix ......................... 1271

Zerbini, Maria Claudia................ 1450, 1451

Zhai, Haiyan.................................... 891

Zhai, Jing................................................... 678

Zhan, Tingting............................... 1952

Zhan, Yougen ............................... 1766 
Zhang, Amy Hong........................... 125

Zhang, Amy J ............................... 484

Zhang, Chao........ 177, 216, 217, 218,

228,229

Zhang, Da........................ 1364, 1495

Zhang, David........................ 842, 1198

Zhang, David Y....... 541, 1317, 1766,

1866

Zhang, Dongwei.......

Zhang, Gloria ... 1100,1280

Zhang, Hailing 1517,1551

Zhang, Hongying ......................... 542

Zhang, Hui ..... 351, 1039, 1704, 1826

Zhang, Japing ................... 1710, 2160

Zhang, Jiexin............................... 1824

Zhang, Jinghui............................. 1890

Zhang, Jiwang ............................. 1476

Zhang, Jun..................................... 900

Zhang, Lei... 33, 55, 841, 1341, 1345,

1612

Zhang, Li........................................ 883

Zhang, Ling............ 1469, 1517, 1553

Zhang, Lingxin........ 839, 1279, 1346,

Zhang, Linsheng ......................... 1550

Zhang, Liying............................... 1234

Zhang, Lizhi.............. 689, 1680, 1782

Zhang, Lun................................ 1537

Zhang, Miao..... 883, 938, 1071, 1087

Zhang, Mingjuan L ............. 375, 2012

Zhang, Pan ........................ 1823, 1848

Zhang, Paul ................................... 598

Zhang, Ping L........ 1511, 1647, 1664, 2027, 2079

Zhang, Pingchuan ....................... 1652

Zhang, Qing ............................. 1257

Zhang, Ranran..... 591, 602, 605, 618,

Zhang, Shanxaing ........................ 1552

Zhang, Shanxiang …........................ 82

Zhang, Shaobo ............................. 1031

Zhang, Sharon S................ 1703, 1711

Zhang, Shengle ............................. 529

Zhang, Shizhen ............................ 883

Zhang, Tong .................................. 802

Zhang, Tuo ..................................... 1857

Zhang, Wei ......................... 842, 2092

Zhang, Wenjing ............................ 1227

Zhang, Xiaofei ... 485, 843, 844, 1801

Zhang, Xiaohui ....... 1517, 1551, 1553

Zhang, Xiaotun ........ 620, 2013, 2014

Zhang, Xuchen... 772, 820, 834, 1713

Zhang, Xuefeng........ 693, 1043, 1701

Zhang, Yang ..................... 1050, 2015

Zhang, Yanhong ............................. 387

Zhang, Yaxia ........ 361, 394, 408, 409

Zhang, Yonghong ........................ 1230

Zhang, Yonghua ................ 2128, 2155

Zhang, Zhang .............................. 542

Zhang, Zhenwei .................... 873, 987

Zhang, Zhiqing.................. 2128, 2155

Zhang, Ziying................................ 410

Zhao, Chengquan ........ 337, 357, 459, $465,486,488,489,1170,1269$

Zhao, Cynthia... ... 487

Zhao, Fang .....

1554

Zhao, Huaqing................. 336, 411, 971

Zhao, Lei ............................ 821, 1885

Zhao, Lili.......................... 1812, 2086

Zhao, Ming............................... 1088

Zhao, Qing
Zhao, Rachel

Zhao, Shengnan......

Zhao, Songqing .................................... 2128

Zhao, Tianhao ............................... 1613

Zhao, Xiaofeng …………………......... 3

Zhao, Xiaohui ................................ 1462

Zhao, Xin ...................................... 357

Zhao, Yani ......................... 1089, 1090

Zhao, Yongxin............................... 121

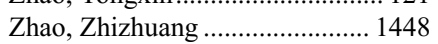

Zhen, Chao J .................................... 629

Zheng, BaoWen........... 488, 489, 1269

Zheng, Gang..................... 1555, 1850

Zheng, Guangjuan....................... 2161

Zheng, Hanqiao............................ 1236

Zheng, Lan ....................... 1374, 1556

Zheng, Qizhi ..................... 853, 993, 2113

Zheng, Shuling ................. 1631, 1653

Zheng, Wenxin ....... 1142, 1224, 1227

Zheng, Xirun ................................ 2161

Zhestkov, Mikhail ........................ 1845

Zhevelyuk, Ekaterina ................... 1845

Zhong, Minghao....... 904, 924, 1023, $1025,1274,1565,1646,1697$, $1765,1820,1866,1871,2147$

Zhou, Amy G ... 402

Zhou, Fang ........................... 343, 397

Zhou, Haijun ......................... 454, 490

Zhou, Jiehao........... 1395, 1500, 1531,

Zhou, Jing ................................. 1440

Zhou, Ming ..... 856, 920, 1000, 1072, 1073, 1089, 1089, 1090

Zhou, Shengmei ............... 1712, 1891

Zhou, Shuang ................................ 102

Zhou, Wenhua ................................... 405

Zhou, Xianrong .............................. 465

Zhou, Xiaoyan..........1111, 1713, 1862

Zhou, Xinchun .................... 847, 1361

Zhou, Yi .................................... 2048

Zhou, Yu........................................ 1607

Zhou, Zhongren........... 623, 805, 1710

Zhu, Daniel .................................. 1686

Zhu, Feng ....................................... 813

Zhu, Hongfa ........ 654, 729, 730, 731, 736, 737, 742, 843, 844, 1801,

1820

Zhu, Menglei.................................. 1091

Zhu, Qingfeng............................... 1707

Zhu, Xiaoqin (Cynthia)................. 1714

Zhu, Yazhen ................................. 2161

Zhu, Yifei …………………….......... 1039

Zhuge, Jian............ 1274, 1563, 1565,

$1765,1866,1871$

Zibadi, Sherma .............................. 816

Zilberg, Catherine ......................... 1305

Ziober, Amy .......................... 709, 1200

Zirpoli, Gary R............................. 1907

Zlobec, Inti.................. 672, 673, 1788

Zlotta, Alexandre R..................... 1070

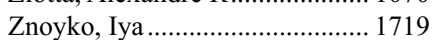

Zoino, Roberto ............................... 860

Zomorrodian, Sina ........... 1732, 1866

Zoroquiain, Pablo... 1731, 1767, 1768

Zou, Youran....................... 1100, 1280

Zreik, Riyam ................. 510, 1168, 1367,

2041

Zsengeller, Zsuzsanna K .................. 9

Zukerberg, Lawrence .................. 1513

Zuo, Chunlai .......... 1005, 1881, 2162
Zuo, Tao

308,1281

Zuo, Zhuang.......... 1355, 1417, 1445,

1452

Zuppan, Craig ................................ 17

zur Hausen, Axel .............................. 509

Zuraski, Connor ................................ 30

Zurbuchen, Emily .......................... 565

Zureikat, Amer H ......................... 1813

Zuretti, Alejandro ......................... 2052

Zutter, Mary M................. 1504, 1510

Zuzga, David................................... 644

Zynger, Debra L .......... 179, 270, 300,

1007,1045 
